\title{
Fault Detection and Diagnosis on the Rolling Element Bearing
}

by

\author{
Aida Rezaei
}

\author{
A thesis submitted to \\ The faculty of Graduate Studies and Research \\ in partial fulfillment of \\ the requirements for the degree of \\ Master of Applied Science \\ Department of Mechanical and Aerospace Engineering \\ Ottawa-Carleton Institute \\ for Mechanical and Aerospace Engineering \\ Carleton University \\ Ottawa, Ontario \\ September 2007
}

Copyright $\bigcirc 2007$, Aida Rezaei 


$\begin{array}{ll}\begin{array}{l}\text { Library and } \\ \text { Archives Canada }\end{array} & \begin{array}{l}\text { Bibliothèque et } \\ \text { Archives Canada }\end{array} \\ \begin{array}{l}\text { Published Heritage } \\ \text { Branch }\end{array} & \begin{array}{l}\text { Direction du } \\ \text { Patrimoine de l'édition }\end{array} \\ \begin{array}{l}\text { 395 Wellington Street } \\ \text { Ottawa ON K1A ON4 }\end{array} & \begin{array}{l}\text { 395, rue Wellington } \\ \text { Ottawa ON K1A ON4 } \\ \text { Canada }\end{array}\end{array}$

Your file Votre référence ISBN: 978-0-494-33665-6 Our file Notre référence ISBN: 978-0-494-33665-6

NOTICE:

The author has granted a nonexclusive license allowing Library and Archives Canada to reproduce, publish, archive, preserve, conserve, communicate to the public by telecommunication or on the Internet, loan, distribute and sell theses worldwide, for commercial or noncommercial purposes, in microform, paper, electronic and/or any other formats.

The author retains copyright ownership and moral rights in this thesis. Neither the thesis nor substantial extracts from it may be printed or otherwise reproduced without the author's permission.
AVIS:

L'auteur a accordé une licence non exclusive permettant à la Bibliothèque et Archives Canada de reproduire, publier, archiver, sauvegarder, conserver, transmettre au public par télécommunication ou par l'Internet, prêter, distribuer et vendre des thèses partout dans le monde, à des fins commerciales ou autres, sur support microforme, papier, électronique et/ou autres formats.

L'auteur conserve la propriété du droit d'auteur et des droits moraux qui protège cette thèse. $\mathrm{Ni}$ la thèse ni des extraits substantiels de celle-ci ne doivent être imprimés ou autrement reproduits sans son autorisation.
In compliance with the Canadian

Privacy Act some supporting forms may have been removed from this thesis.

While these forms may be included in the document page count, their removal does not represent any loss of content from the thesis.
Conformément à la loi canadienne sur la protection de la vie privée, quelques formulaires secondaires ont été enlevés de cette thèse.

Bien que ces formulaires aient inclus dans la pagination, il n'y aura aucun contenu manquant.

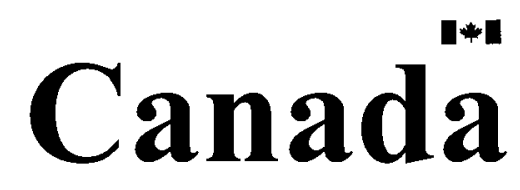




\begin{abstract}
The use of novel sensors such as Air-coupled ultrasonic transducer, Eddy current, Piezoelecteric ultrasonic transducer, and piezo strain gage as diagnostic tools for detection of bearing faults have been investigated. A series of experiments was carried out in a laboratory environment. Localized defects with different sizes were created intentionally on the test bearing components simulating evolving cracks or other related faults. Four different signal processing techniques were applied to extract the signal features .The resulting data for different bearing speed and load showed that the sensors are capable of detecting different types of defects located on the bearing components. The data from the sensors were also compared with those obtained from accelerometers. Then, a practical classification model based on a decision tree algorithm was developed and applied on the extracted features. The results showed that the defect could be detected and classified at an early stage on each component of the bearing.
\end{abstract}




\section{Acknowledgments}

I wish to express my sincere appreciation to Tribology Group of Gas Turbine Laboratory of the National Research Council of Canada for providing the opportunity for me to participate in this interesting project. I am really indebted to Dr.Waldek Dmochowski, Jeff Bird, Dr.Sylvian Letourneau, Brian Liko, John William, Randy payette, and Martin Murach who contributed their experience and knowledge in this project. Dr. Azzedine Dadouche was my valuable guider and co-supervisor with Professor Fred Nietzsche throughout this research. I truly appreciate their tremendous support and help. It is especially important to thank Viresh Wickramasinghe for his invaluable collaboration with this project.

I would like also to thank Professor Susan Logie for her constructive criticisms and suggestions in the preparation of the report.

I give thanks to my parents for their love and patience. The last but not the least, a world of thanks goes to my friend and husband, Behnam, for all his enthusiasm and support. 


\section{Table of Contents}

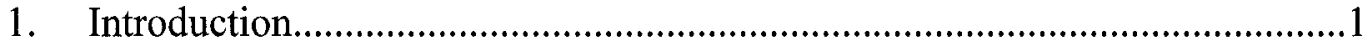

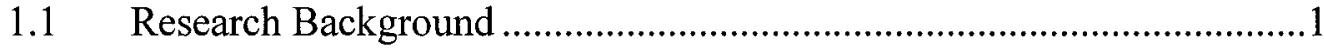

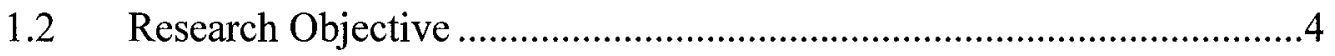

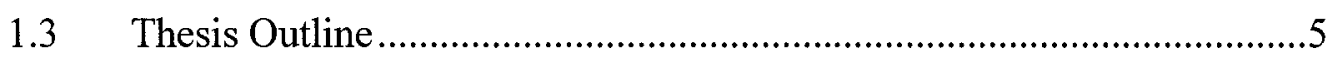

2 Bearing Failure Types and Fault Signatures..................................................

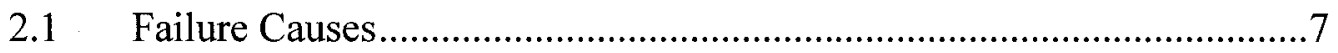

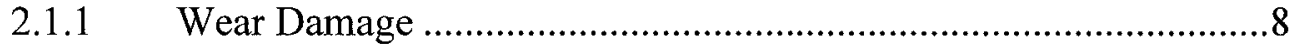

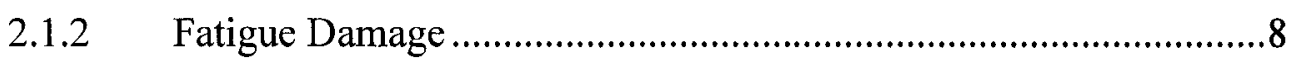

2.1.3 Corrosion Damage ..............................................................

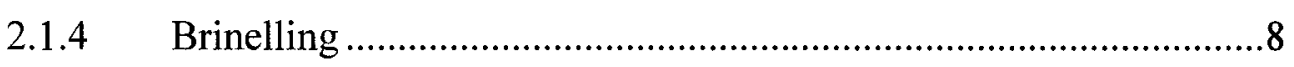

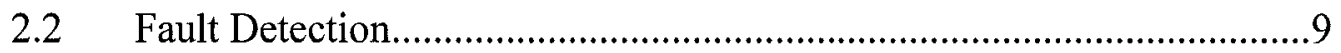

2.2.1 Thermal Analysis ..................................................................

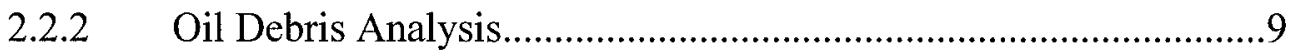

2.2.3 Vibration Analysis .....................................................................

2.3 On-line Monitoring and Health Management.......................................11

2.4 Fault Signatures and Characteristic Frequencies ....................................12

2.4.1 Cage Frequency ………………………………........................12

2.4.2 Outer/Inner Race Ball Pass Frequency …………….......................14

2.4.3 Ball Spin Frequency …………...............................................

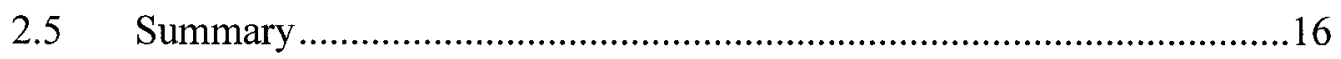

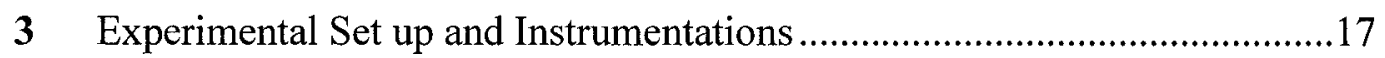

3.1 Test Rig Design .............................................................................17

3.1.1 Frame Assembly ...............................................................18

3.1.2 Test Bearing Assembly ..........................................................19

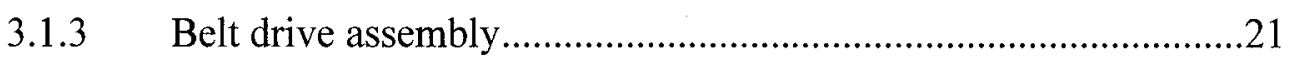

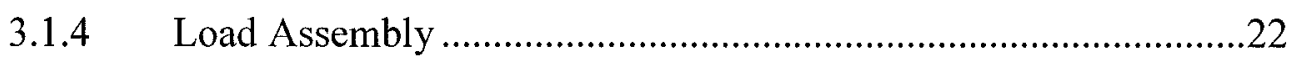

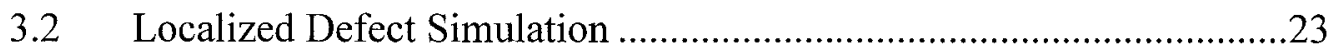

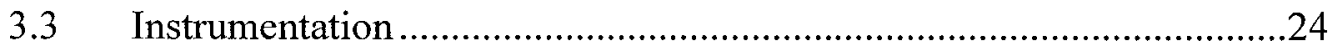

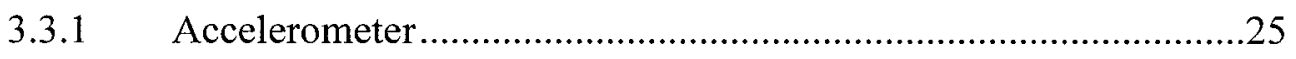

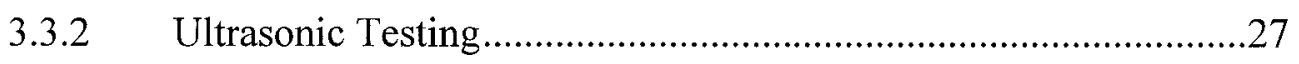

3.3.3 Eddy Current Sensor......................................................................33

3.3.4 Piezoelectric Strain Gage ...........................................................34

$3.4 \quad$ Sampling Frequency Determination .......................................................36

3.4.1 Impact Tests......................................................................... 
3.5 Vibration and Acoustic Test Procedures .............................................40

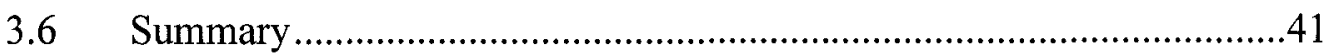

4 Vibration Analysis Techniques on Rolling Elements......................................42

$4.1 \quad$ Time-Domain Analysis...................................................................42

4.1.1 Waveform Analysis ...................................................................43

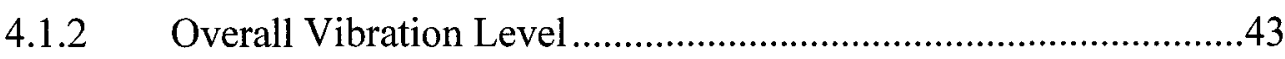

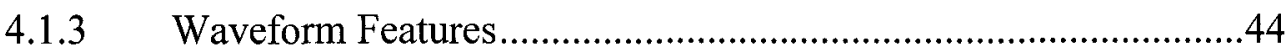

4.2 Frequency Analysis ......................................................................

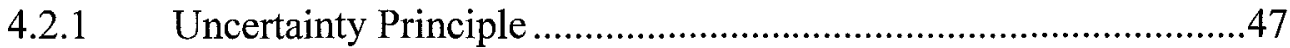

4.2.2 Spectral Analysis .....................................................................48

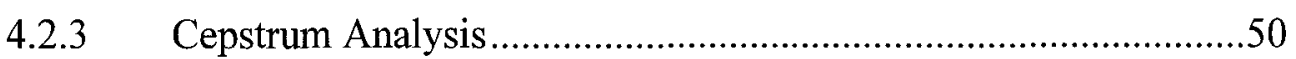

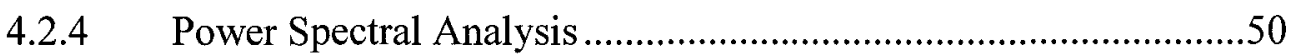

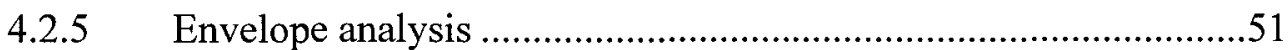

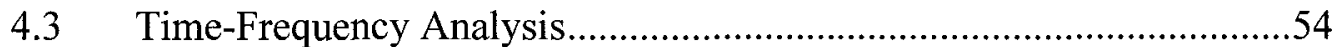

4.3.1 Short Time Frequency Transform ..............................................54

4.3.2 Wavelet transform …………………………….....................56

4.3.3 Envelope Spectra of Wavelet Transform.......................................60

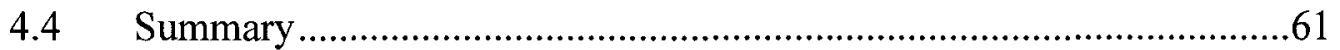

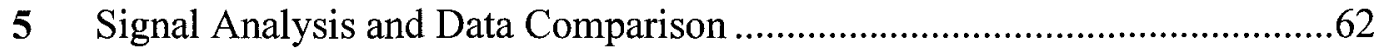

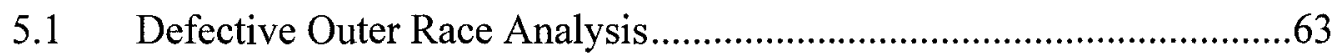

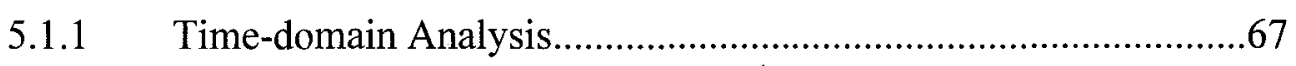

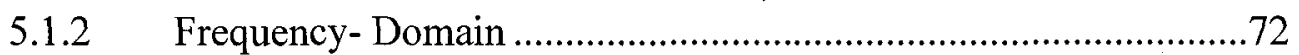

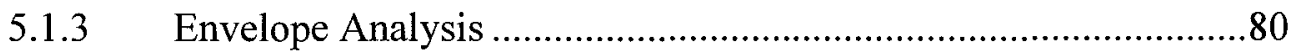

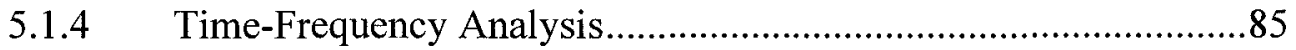

5.1.5 Envelope Spectrum of Wavelet Transform ..................................90

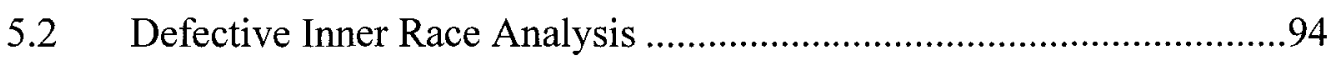

5.2.1 Time-Domain Analysis................................................................95

5.2.2 Frequency-Domain Analysis .....................................................97

5.2.3 Time-Frequency Analysis........................................................101

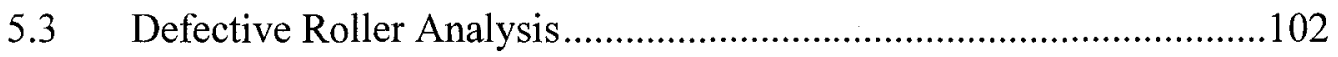

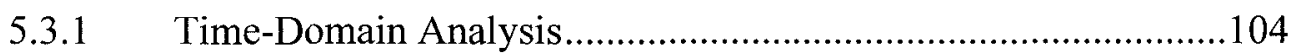

5.3.2 Frequency-Domain Analysis ...................................................105

5.3.3 Frequency-Time Domain Analysis.............................................109

5.4 Conclusion remarks .....................................................................

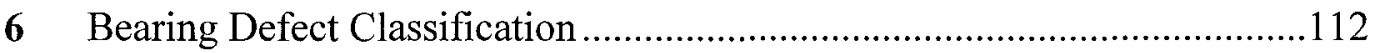

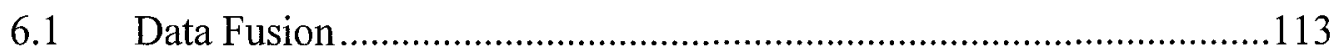




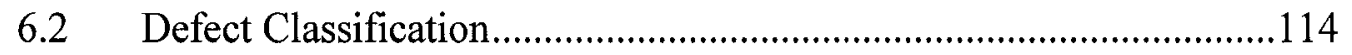

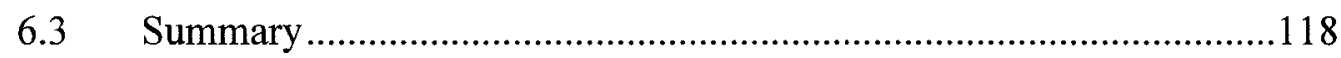

7 Conclusions and Future work .............................................................119

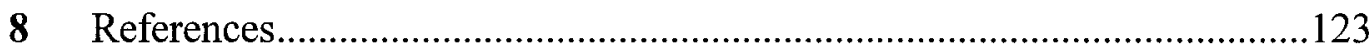

A. Appendix A: Axial Accelerometer Time and Frequency-Domains ............129 


\section{List of Figures}

Figure 2.1 Geometry of a bearing ......................................................................14

Figure 3.1 Bearing test rig with various assemblies [Tribology group, NRC] ......18

Figure 3.2 Test bearing assembly ...................................................................20

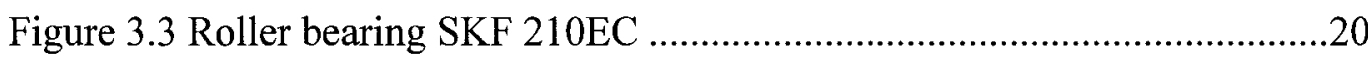

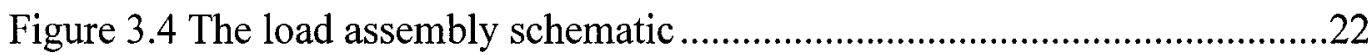

Figure 3.5 Created bearing defects, length of $10 \mathrm{~mm}$ and width of $1 \mathrm{~mm}$..............23

Figure 3.6 (a) ACUT set up for test bearing,(b) and (c) The capacitive ultrasonic .30

Figure 3.7 Internal View of PUT ...................................................................

Figure 3.8 Piezoelectric ultrasonic sensor on bearing ..........................................32

Figure 3.9 Eddy current probe and Zetek unit ......................................................34

Figure 3.10 Piezoelectric strain gage on the beam attached to the bearing............36

Figure 3.11 Test fixture for impact test on the roller bearing.................................37

Figure 3.12 FRF of the rolling bearing, Accelerometer on the outer race..............39

Figure 3.13 FRF of the roller bearing, Accelerometer on the inner race.................39

Figure 4.1 Time and frequency resolutions of the STFT ......................................56

Figure 4.2 Time and frequency resolutions of the wavelet transform....................58

Figure 4.3(a)Original Mex.hat, (b)Translated Mex.hat, $(\tau=2)$,(c) dilated Mex.hat ( $(\mathrm{s}=2)$,

(d) Translated and scaled Mex.hat $(\tau=2, \mathrm{~s}=0.2) \ldots \ldots \ldots \ldots \ldots \ldots \ldots \ldots \ldots \ldots \ldots \ldots \ldots \ldots \ldots \ldots . . .59$

Figure 5.1 Typical radial accelerometer signal for outer race, 1800rpm, $500 \mathrm{lbf} . .64$

Figure 5.2 Typical ACUT signal for outer race, 1800 rpm, 500lbf.........................64

Figure 5.3 Typical Eddy current signal for outer race, 1800rpm, 500lbf...............65

Figure 5.4 Typical PUT signal for outer race, 1800rpm, 500lbf ...........................65

Figure 5.5 Typical accelerometer signal for defective outer race, $1800 \mathrm{rpm}, 500 \mathrm{lbf}$ . .66

Figure 5.6 Typical ACUT signal for defective outer race, 1800rpm, 500lbf ........66

Figure 5.7 Accelerometer peak values for outer race defects.................................67

Figure 5.8 Accelerometer RMS values for outer race defects................................68

Figure 5.9 Accelerometer crest factor for outer race defects.................................68

Figure 5.10 ACUT RMS Value for outer race defects .........................................69

Figure 5.11 ACUT RMS values for outer race defects ..........................................70

Figure 5.12 PUT Peak value for outer race defects ..............................................71

Figure 5.13 PUT RMS value for outer race defects .............................................. 
Figure 5.14 Accelerometer spectra of outer race, $1800 \mathrm{rpm}, 500 \mathrm{lbf}$ .72

Figure 5.15 Acceleration spectra of outer race, $1800 \mathrm{rpm}, 500 \mathrm{lbf}$, low- frequency range 73

Figure 5.16 Effect of load and speed on radial vibration amplitude ......................74

Figure 5.17 ACUT signal of outer race, 1800 rpm, 5001bf ...................................75

Figure 5.18 ACUT Spectra of outer race, $1800 \mathrm{rpm}$, 500lbf, low frequency range

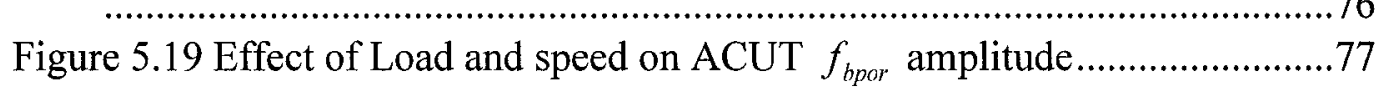

Figure 5.20 Eddy current spectra of outer race, 1800rpm, 500lbf..........................78

Figure 5.21 Eddy current spectra of outer race, 1800rpm, 500lbf..........................78

Figure 5.22 PUT signal spectra of outer race, 1800rpm, 500lbf, low- frequency

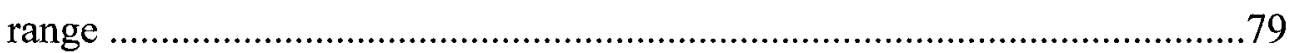

Figure 5.23 Effect of Load and speed on PUT, $f_{\text {bpor }}$ amplitude ............................80

Figure 5.24 Accelerometer demodulated signal spectra of outer race,...................81

Figure 5.25 ACUT demodulated signal spectra of outer race, 1800rpm, 500lbf ..82

Figure 5.26 Eddy current demodulated signal spectra of outer race, .....................83

Figure 5.27 Strain gage, defective outer race, 1800rpm, 500lbf, ........................84

Figure 5.28 The $7^{\text {th }}$ derivative of the complex Gaussian function characteristics.86

Figure 5.29 Wavelet analysis of defective outer race, radial accelerometer, ........88

Figure 5.30 Wavelet analysis of defective outer race, ACUT, .............................89

Figure 5.31 Wavelet-based envelop spectrum of healthy bearing, ........................91

Figure 5.32 Wavelet-based envelop spectrum of defective outer race, 1800rpm .91

Figure 5.33 Wavelet-based envelope spectrum of defective outer race, $1800 \mathrm{rpm}$,

Figure 5.34 Wavelet-based envelop spectrum of defective outer race, ,..............92

Figure 5.35 Wavelet-based envelope spectrum of healthy bearing, $1800 \mathrm{rpm}$,

500lbf, acoustic signature (ACUT) ..............................................................93

Figure 5.36 Wavelet-based envelope spectrum of defective outer race, 1800rpm, 500lbf, acoustic signature (ACUT), defect width of $1 \mathrm{~mm}$........................93

Figure 5.37 Accelerometer time history signal of inner race, 1800rpm, 500lbf ...94

Figure 5.38 ACUT time history signal of inner race, 1800rpm, 500lbf ................95

Figure 5.39 Defect detectability factors versus defect size, defective inner race,

1800 rpm, 500lbf, vibration signature ...........................................................96

Figure 5.40 Defect detectability factors versus defect size, defective inner race, 1800rpm, 500lbf, acoustic signature (ACUT) ……………………………....97

Figure 5.41 Vibration signature spectra of inner race, 1800rpm, 500lbf ..............98 
Figure 5.42 Acoustic signature (ACUT) spectra of inner race, $1800 \mathrm{rpm}, 500 \mathrm{lbf} 98$ Figure 5.43 Acoustic signature (PUT) spectra of inner race, 1800rpm, $500 \mathrm{lbf}$...99

Figure 5.44 Demodulated vibration signature spectra of inner race,...................100

Figure 5.45 Demodulated acoustic signature (ACUT) spectra of inner race, .....100

Figure 5.46 Demodulated eddy current signal spectrum of inner race, ..............101

Figure 5.47 Wavelet-based envelope spectrum of defective inner race, 1800rpm, 102

Figure 5.48 Accelerometer time history of roller, 1800rpm, 500lbf ..................103

Figure 5.49 ACUT time history of roller, 1800rpm, 500lbf............................103

Figure 5.50 Defect detectability, vibration signature, roller,..............................104

Figure 5.51 Defect detectability, acoustic signature (ACUT), roller, ................ 105

Figure 5.52 Vibration signature spectra of roller, 1800rpm, 500lbf...................106

Figure 5.53 Acoustic signature (ACUT) spectra of roller, 1800rpm, 500lbf ......106

Figure 5.54 Acoustic signature (PUT) spectra of roller, 1800rpm, 500lbf...........107

Figure 5.55 Demodulated vibration signature spectra of roller, ........................108

Figure 5.56 Demodulated acoustic signature (ACUT) spectra of roller,.............108

Figure 5.57 Demodulated Eddy current signal spectra of roller,......................109

Figure 5.58 Wavelet-based envelope spectra of defective roller, 1800rpm, .......110

Figure 6.1 Classification method for fault diagnosis.......................................113

Figure A.1 Axial accelerometer signal for outer race, 1800rpm, 500lbf.............129

Figure A.2 Axial accelerometer spectra of outer race, 1800rpm, 500lbf ............129

Figure A.3 Accelerometer demodulated signal spectra of outer race,.................130 


\section{List of Tables}

Table 3.1 Test bearing dimensions ..............................................................21

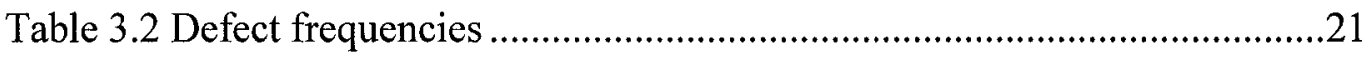

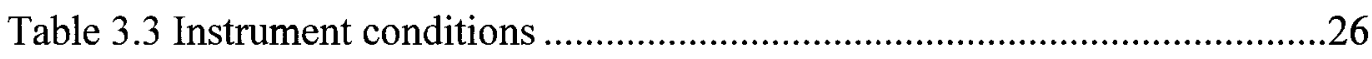

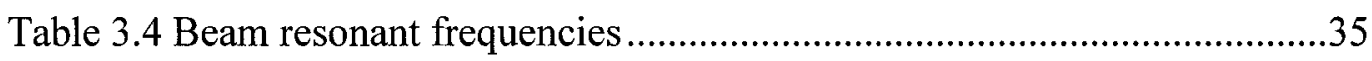

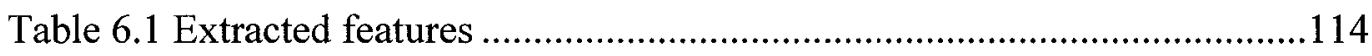

Table 6.2 Classification results using a radial accelerometer ............................117

Table 6.3 Performance results ..............................................................118 


\section{Nomenclatures}

Symbols

$\alpha \quad$ Pitch angle

$\tau \quad$ Translation

$\omega \quad$ Angular Velocity

$\psi \quad$ Mother Wavelet

A Arbitrary constant

$A_{c} \quad$ Amplitude of Fourier transform of wavelet coefficient

C Cepstrum

d Pitch diameter

D Race diameter

E Energy Density Spectrum

L Load

f Frequency

S Scale

$t \quad$ Time

$x \quad$ Signal history in time/ frequency -domain

V Velocity

$W_{c} \quad$ Wavelet coefficients

$\gamma \quad$ Covariance function

$P \quad$ Power spectrum density

g Complex Gaussian function

$S \quad$ Short time Fourier transform

$M W_{c} \quad$ Modulus of wavelet coefficients

$N \quad$ Number of data points

Z Number of rollers 
Acronyms

$\begin{array}{cl}\text { ACUT } & \text { Air Coupled Ultrasonic Transducer } \\ \text { Bl } & \text { Baseline } \\ \text { Ca } & \text { Cage } \\ \text { DFT } & \text { Discrete Fourier Transform } \\ \text { FFT } & \text { Fast Fourier Transform } \\ \text { Ir } & \text { Inner race } \\ \text { Mex.hat } & \text { Mexican hat } \\ \text { Or } & \text { Outer race } \\ \text { PUT } & \text { Piezoelectric Ultrasonic Transducer } \\ \text { RMS } & \text { Root Mean Square } \\ \text { Ro } & \text { Roller } \\ \text { S } & \text { Sensor } \\ \text { STFT } & \text { Short Time Fourier Transform }\end{array}$




\section{Chapter 1}

\section{Introduction}

\subsection{Research Background}

Mechanical failure prevention and condition monitoring have been one of the mechanical engineers' concerns in recent years due to the personal safety, reliability, failure cost, and equipment downtime issues. Proper system failure prevention process helps to reduce the possibility of the system malfunction, identification of source causes, and troubleshooting. Generally, failure prevention can be identified as the process of fault detection, diagnosis, and prognosis:

i. Fault detection is the process of observing the measured system data and system status information and comparing them with a normal range of observed attributes to determine whether some measurements fall outside the range representing the healthy condition of the system

ii. Diagnosis is the process of determining the state of failing components, and identifying the cause(s) of the failure

iii. Prognosis is the process of predicting impending component failures or abnormal system states before they actually occur, and estimates their remaining useful life.

The required degree of fault prevention depends strongly on the complexity of the system and the application. For example, it is important for the rotating machinery used in the aerospace industry to perform fault detection, diagnosis with different means as well as prognosis to predict the time of failure of the 
critical components.

Bearings are among the most critical mechanical components that have wide applications in many industries and have proven to be reliable and long-lived when properly applied. As a result of improvements in bearing materials, design, lubrication technology and service life, they have been gradually employed under more severe application requirements such as higher load, higher speed, and restricted lubrication. These requirements have made condition monitoring and fault diagnosis of bearings very important to ensure safe operation of rotary machines.

Bearing failure prevention through health monitoring have been one the most researched areas in the past decades in the field of mechanical engineering. These research activities have resulted in a better understanding of bearing health analysis as well as advanced diagnostic methodologies (Howard, 1994).

Although several oil debris monitoring methodologies have shown some satisfactory applications on bearing health monitoring, the most successful methods in use at present are all based on vibration analysis. Vibration analysis is performed through the extraction of features from a sensor signal and associating these features with healthy or faulty components of the bearing. More advanced bearing heath monitoring methods using vibration could even determine the defect severity. Recent advancement in cheap and powerful computers has enabled the use of advanced signal processing and analysis techniques required for improved condition monitoring of the bearing.

The task of condition monitoring of rolling element bearing is often burdensome, labor intensive and complex procedure because of:

- The location of defect, which generates different dynamic behavior,

- Existence of high level of noises in the rest of the system,

- The variation of load and shaft speed during operation, 
- The tolerances of assembly and geometry during any operation.

However, an appropriate choice of the sensors and applicable signal processing techniques along with a good test procedure facilitates to overcome the difficulty of condition monitoring of rolling element bearing.

The early practical works on vibration diagnostics were based on the commercial real-time spectral analyzers. Then, various attempts have been made to analyze vibration resulting from faults on machines using the spectral analyses methodologies (White (1972) and Swansson (1984)). By the end of the 1980s, the vibration analyses of faulty rotating machines were based on either the time domain analyses or frequency domain analyses. Forrest (1989) showed the nonstationary nature of the signals extracted from a defective rotating component and the capability of the joint time-frequency technique to detect the defects. In addition, Cohen (1989) reviewed the time-frequency techniques and their applications.

An excellent literature review that highlights several techniques and vibration sensors for fault diagnosis and condition monitoring of localized bearing faults was written by Howard (1994). In his report he explains the different modes of failure, described kinematics and dynamics in rolling bearings and summarizes the available signal processing techniques at the time of publication.

Dadouche and Bird (2005) provide an overview of techniques and sensors applied for rolling bearing diagnosis, over the last decade. They also showed that, although the literature is not lacking in identification of applied techniques, there is still a void in examining a number of signal processing techniques on some of the more novel and useful sensors.

However, a practical widely applicable mechanical bearing monitoring system is still to be developed and accomplishment of such a system requires new ideas as well as new implementation concepts to increase the system sensitivity and achieve true prognosis capability. 


\subsection{Research Objective}

This thesis is a report of a study of techniques for fault detection and diagnosis on rolling bearing elements based on vibration and acoustic analyses. The primary objective of this research thesis is to identify the capability of novel sensors through experimental evaluations and apply suitable techniques to diagnose defects on the bearings.

The secondary objective is to present a comparative study of four representative bearing vibration analysis techniques commonly employed for non-stationary signal processing. These four techniques include time domain, frequency domain and two of joint time-frequency domain analyses. Because each of the presented techniques has shown to be successful in extracting features hidden within the signals, a proportional evaluation of these techniques provides a useful direction to select the most effective technique for the bearing health management applications. Thus, this thesis investigates these four representative techniques in detail to diagnose faulty bearings.

The final objective is to evaluate a classification methodology to determine the bearing as healthy or defective and identify the type of defect(s) as well as the level of the defect severity. This classification was achieved to some extent by appropriate feature extraction from vibration signals and data fusion of the sensors.

The flow diagram shown in Figure 1.1 provide an overview of the procedure followed in this research activity, that included data acquisition from vibration tests with variety of sensors, develop methodologies to analyze the data and perform classification based on the data that leads to the diagnosis of a faulty bearing.

Although this particular research activity focused on the rolling element bearings, the research process could be successfully used for analyses of gearboxes and 
spindles.

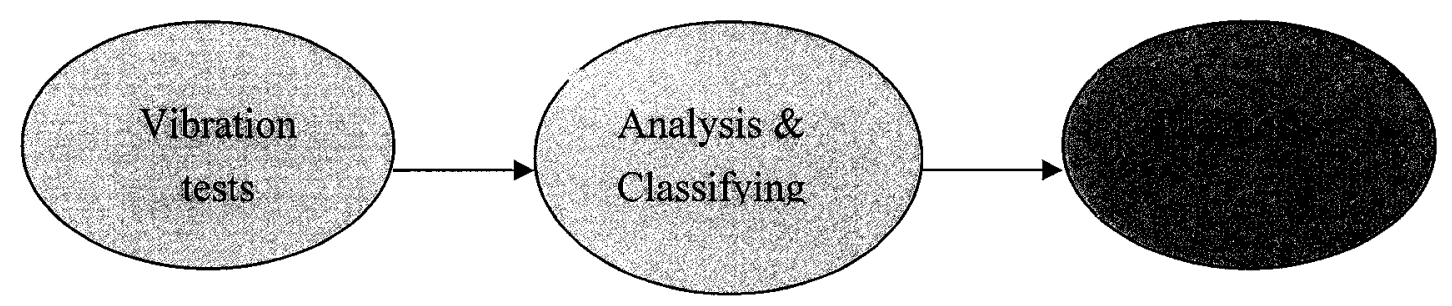

Figure 1.1 The simplified procedure of the thesis tasks

\subsection{Thesis Outline}

The chapters in this thesis are organized in the following manner:

Chapter two, "Bearing Failure Types and Fault Signatures", is a summarized review of failure modes of bearings and existing fault detection techniques and also provides a model of the vibration signature generated by the bearing operation

Chapter three, "Experimental Set up and Instrumentation", describes the development of the designed experimental test set up, the generation of seeded faults, the applied sensors, and the measuring conditions

Chapter four, "Vibration Analysis Techniques on Rolling Elements" reviews the investigated vibration analyses techniques and signal processing methods in order evaluate which methods are the most suited to the detection and diagnosis of the failure modes.

Chapter five, "Signal Analyses and Data Comparison", performs analyses based on time, frequency, and time-frequency techniques explained in Chapter four using data collected from different sensors described in Chapter three.

Chapter six, "Bearing Fault Classification", attempts to fuse the results described 
in the Chapter five in order to feed to a classifier that could identify the condition of the bearing,

Chapter seven, "Conclusions and Future Work", provides concluding remarks based on the performed research activity and identifies potential for further research in order to advance the technical knowledge in this field. 


\section{Chapter 2}

\section{Bearing Failure Types and Fault Signatures}

In this Chapter, the causes of bearing failures, their probable effects, methods which can be used to reduce bearing failures and a review of processes involved in generation of vibration from a defective bearing will be addressed.

\subsection{Failure Causes}

Bearing failure can cause not only personal injury but also unscheduled replacement or repairs, which lead to high maintenance costs in rotating machinery. To list, all the known types and causes of bearing damage is beyond the scope of this thesis and for this reason only the most common bearing failure causes are mentioned.

Wear, crack, fatigue, corrosion, and brinelling are the most important failure means, which cause localized defects on bearings. The other types of failures which cause distributed defects generally originate from inadequate maintenance, improper disassembling or assembling; and harsh operating conditions such as insufficient lubrication or very high speed or acceleration (Harris, 2000) and (Wiley, 1999).

The performance characteristics of a defective bearing can be monitored by variety of measurement instrumentations through processes such as vibration or temperature analyses. At the first signs of the bearing damage, the causes and effects should be analyzed so that the steps to avoid the further damage can be taken. 


\subsubsection{Wear Damage}

Wear is a frequent cause of bearing damage. Wear occurs mainly due to dirt and foreign particles entering the bearing through inadequate sealing or contaminated lubrication, which result in an increase in friction between metal contacts, and changes of the raceway profile. The exposed bearing to the wear damage would gradually deteriorate leading to a loss of dimensions and associated problems.

\subsubsection{Fatigue Damage}

After a certain running time, a bearing that is subjected to loading fails due to fatigue of the material. If a bearing is also destructively preloaded or overstressed, after a shorter operating time, it will also stop working due to fatigue damage. A fatigue crack begins below the surface and propagates towards the surface as loading continues, until a piece of metal breaks away leaving a pit in the contact area. Fatigue grows faster if the bearing is overloaded, over speeding, or oil starving. These conditions severely reduce the service life of bearings and they are normal occurrences in all bearings (Harris, 2000). If a bearing fails due to fatigue sooner than its predicted time, the failure can generally traced to either overloading or bad installation or maintenance.

\subsubsection{Corrosion Damage}

The rust pits caused by corrosion on a bearing element results in excessive noise during operation. The rust generates when the bearing is exposed to water, acid, acidic lubrication, or exposure to elements due to incorrect storage. Condensation is another cause of corrosion on a bearing. Condensation is caused by sudden cooling of the bearing from operating temperature in humid air. Condensation may even damage bearings prior to installation.

\subsubsection{Brinelling}

Permanent indention created by rolling element overload is called brinelling. The 
indentions may result from static loading, which leads to observable plastic deformation of the raceways. Similar damage may occur while a stationary rolling bearing is exposed to vibration and shock loads. When the lubricant is derived out of a loaded or vibrated region, the indentions and wear appear to mimics brinelling. Brinelling is evident in the raceways through the indents or wear and can increase bearing noise and vibration, leading to premature bearing failure.

\subsection{Fault Detection}

Detecting mechanical faults in bearings has been recognized for some time as an important aspect for preventing catastrophic failure and planning effective maintenance.

There are several approaches used to diagnose faults on a bearing system including thermal analysis, oil debris analysis, and vibration analysis.

\subsubsection{Thermal Analysis}

Thermal analysis is defined as a tool used to generate warnings about the overheating of the bearing system. The thermocouples or other temperature monitoring devices usually employed at the inlet and outlet of the test chamber indicate two points of interest to study any temperature gradient of the bearing component. However, thermal analysis cannot be used to identify the type and size of the defects in a bearing system.

\subsubsection{Oil Debris Analysis}

Lubrication of a bearing may be provided in liquid, grease, or solid form and the type of lubrication is generally chosen depending on the operating conditions. Debris analysis does have a rare application on grease or solid form but generally used with oil lubricants. Bearing failures in some machines such as helicopter transmissions and aircraft turbine engines generate significant debris in their oil systems. Oil analysis program on a bearing consists of oil sampling, analytical 
tests and data interpretation. There are number of oil debris analysis techniques such as elementary spectroscopy, wear particle analysis, fine particulates analysis, molecular analysis, and electrochemical chemistry used to diagnose a failure on a bearing. These oil debris analyses provide information on quantity, form, and size distribution of the debris, which can lead to damage type detection.

Research (Akagaki et al, 2006) has shown that small amounts of oil debris extracted from oil analysis can reveal the severity of rolling-contact fatigue wear. In their study, the presence of large particles was considered a supporting symptom for assessing the wear severity levels. Wear particles were considered to be a critical indication that a bearing needed to be changed before a forced outage occurred. However this type of analysis could not differentiate between the damaged components of a bearing because of the material similarity.

Practical experiences and researches have shown that combining oil debris analysis along with vibration analysis in a bearing condition-monitoring program provides information in greater detail and more reliable information than each individual system.

\subsubsection{Vibration Analysis}

Each rotating machine has its own vibration signature as a result of the rotation of shafts, gears and bearings. Rolling bearing elements, an essential part of rotating machinery, are known to play a significant role in machine vibrations. First, structural element of the bearing acts as a spring and also adds some mass to a system. As such, bearings define, in part, the vibration response of the system to external time-varying forces. Secondly, bearings act as excitation forces, producing time-varying forces that cause system vibration. This excitation is natural in the design of rolling bearings. However, these forces can be greatly amplified as a result from imperfections or defects on the bearing components.

Detection of progressive bearing deterioration during operation by vibration measurements has been in use for a long time and this technique has become more economical and reliable in recent years. The overall level of vibration indicates 
the general condition of the bearing system, the cause of vibration, including such factors as unbalance, misalignment, and bearing defects. Vibration diagnostics are usually concerned with the extraction of features from a signal and associating these features with healthy or faulty components of the bearing.

A healthy bearing under constant load and speed is likely to move toward steady state dynamic equilibrium, because of the natural symmetry in a rolling bearing element. The strength of the vibration increases when a defect occurs on one of the bearing components. Due to the defect, a transient force takes place each time another bearing component contacts the defective surface, resulting in rapid acceleration of the bearing components. Vibration analysis technique will be discussed in detail in the next chapter and explained further in the remainder of this thesis.

\subsection{On-line Monitoring and Health Management}

On-line or continuous monitoring systems are generally used to continuously monitor the potential bearing failures. By upgrading a rotary machine with a fault prediction system, the machine can continue performing within acceptable parameters and be repaired at the time most economically convenient to the plant. Adding prediction capability to the monitoring system is the difference between simply saving the machine itself and saving the production schedule as well.

Protection of the bearings in rotary machines through on-line monitoring systems offers machine operators the ability to reduce maintenance costs, increase machinery availability (lower downtime) and avoid catastrophic failure. To provide on-line (computerized) analysis of the bearing health, portable multiple data acquisition collectors are used to gather data with variety of sensors from different points on the system. The advanced diagnostic software help operators to identify fault signature before bearings reach a predictable alarm.

A structure connected to fault detection, fault diagnosis, fault prognosis and control system is known as a health management or monitoring system. It employs several data analysis and decision making techniques including statistical 
analysis, data visualizations, and discriminant analysis, in order to perform data mining, feature extraction, fault detection and diagnosis. The health management system can be designed to work on-line or off- line on the desired system.

\subsection{Fault Signatures and Characteristic Frequencies}

When a single component of a bearing is defected because of the one of mentioned failure causes, it is simple to identify the fault signature generated by the bearing. Each time one of the rollers (balls) rolls over the defect, an impulsive force occurs that causes the whole system of the bearing to vibrate. The bearing responds by "ringing" at its natural frequency, a response that decays quickly because of damping in the structural system. This response happens each time one of the rollers rolls over the defect. Therefore, the fundamental frequency of the response waveforms is the rate at which the component rolls over the defect and this frequency is of interest in the detection of bearing faults. Each defect is characterized by a specific defect frequency or characteristic frequency, depending on which bearing component the defect occurs, the bearing geometry, and the speeds at which the inner and outer races rotate. Different frequencies are generally obtained for defects on an outer race, inner race, on one of the rollers. The equations for ball passing frequencies, ball spinning frequency, and cage frequency give the characteristic bearing frequencies (Berry, 1991). These equations do not account for skidding, which is neglected at low speeds and in cases of high loads.

\subsubsection{Cage Frequency}

The velocity of a rolling element due to rotation about its own axis is equal and opposite at the point of the contact with the inner and outer races. Hence, the axes of the rolling elements and the cage holding the rolling elements must move with a velocity equal to the mean of the velocities of the inner and outer races in order to maintain the races without the sliding. 


$$
V_{c a}=\frac{V_{o}+V_{i}}{2}
$$

where $V_{c a}$ is the tangential velocity at the pitch circle and $V_{i}$ and $V_{o}$ are the velocity of the inner and outer race respectively. To convert the equation (2-1) to rotational frequency based on the geometry of the bearing (Figure (2-1)):

$$
f_{c a}=\frac{D_{o} f_{o}+D_{i} f_{i}}{2 D}
$$

where:

$f_{o}$ - The outer race frequency, $f_{i}$ - The inner race operating frequency

while, the relationship between the inner race and outer race diameter with the pitch circle diameters, $D$ the rolling element diameter, $d$ and contact angle, $\alpha$ are as follows:

$$
\begin{aligned}
& D_{o}=D+d \cos (\alpha) \\
& D_{i}=D-d \cos (\alpha)
\end{aligned}
$$




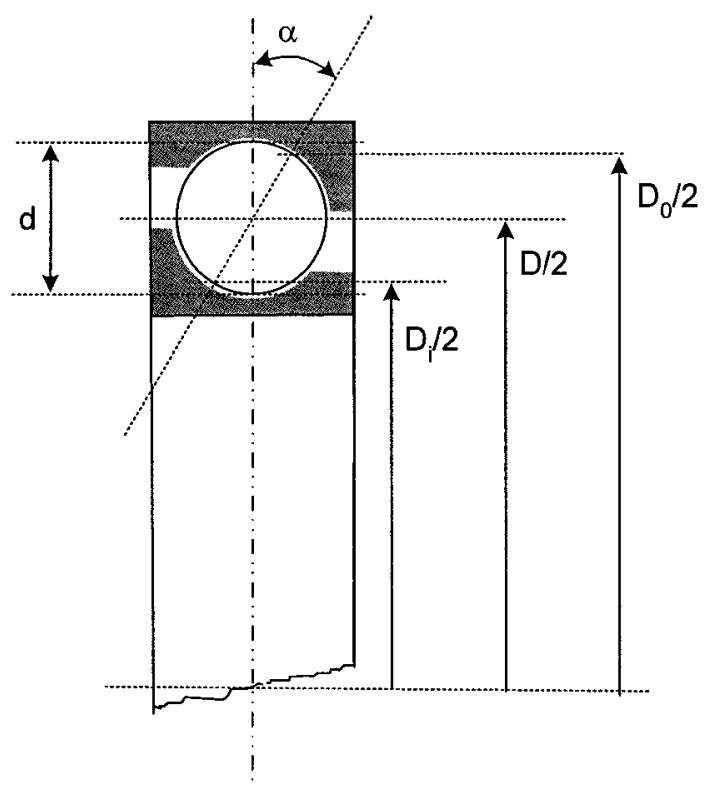

Figure 2.1 Geometry of a bearing

To substitute equations (2-3) and (2-4) into equation (2-2), the rotational frequency of cage can be calculated by considering races diameter, pitch angle and rolling element diameter as follows:

$$
f_{c a}=\frac{\left(1+\frac{d}{D} \cos (\alpha)\right) f_{o}+\left(1-\frac{d}{D} \cos (\alpha)\right) f_{i}}{2}
$$

\subsubsection{Outer/Inner Race Ball Pass Frequency}

The inner and outer races of a bearing are attached to other component. Usually, the inner race is attached to the shaft and thus has the same rotational speed as the shaft and the outer race is stationary. The inner and outer race defect frequencies are referred to as ball pass frequencies. The ball pass frequencies of inner race and outer race can be calculated with the following equations (Harris, 2000): 


$$
\begin{aligned}
& f_{b p i r}=z\left(f_{c a}-f_{i}\right)=\frac{z}{2}\left(f_{i}-f_{o}\right)\left(1+\frac{d}{D} \cos (\alpha)\right) \\
& f_{b p o r}=z\left(f_{c a}-f_{o}\right)=\frac{z}{2}\left(f_{i}-f_{o}\right)\left(1-\frac{d}{D} \cos (\alpha)\right)
\end{aligned}
$$

where,

$z$ - The number of rollers (ball elements).

\subsubsection{Ball Spin Frequency}

The ball defect frequency is usually referred to as the ball spin frequency. Ball spin frequency is the frequency at which the roller (ball element) makes contact with one of the races. The ball spin frequency equation is calculated based on the equal time that a roller (ball element) takes to go across a distance equal to its diameter, $d$ along with the inner or outer race.

$$
\begin{aligned}
& f_{r o} \pi d=\left(f_{i}-f_{c a}\right) \pi(D-d \cos (\alpha))=\left(f_{o}-f_{c a}\right) \pi(D+d \cos (\alpha)) \\
& f_{r o}=\frac{1}{2}\left(f_{i}-f_{o}\right) \frac{D}{d}\left(\left(1-\frac{d}{D} \cos (\alpha)\right)^{2}\right)
\end{aligned}
$$

It should be noted that equations (2-6), (2-7), and (2-9) do not account for skidding of the rollers in the bearing

With Assuming fixed outer race, moving inner race with shaft speed, $f_{s}$, and no slippage for the rolling, equations of (2-6), (2-7), and (2-9) are as follows: 
$f_{b p i r}=\frac{z}{2} f_{s}\left(1+\frac{d}{D} \cos (\alpha)\right)$

$f_{\text {bpor }}=\frac{z}{2} f_{s}\left(1-\frac{d}{D} \cos (\alpha)\right)$

$f_{r o}=\frac{1}{2} f_{s} \frac{D}{d}\left(\left(1-\frac{d}{D} \cos (\alpha)\right)^{2}\right)$

\subsection{Summary}

In this chapter, the failure modes of rolling bearing elements, the causes, and the commonly used techniques to diagnose the defect on the bearings were reviewed. Vibration analysis has been shown to be the most effective tool among the available techniques to analyze bearing failure. The role of the characteristic frequency in vibration analysis has been examined. In the next chapter, the experimental set up to simulate a failure mode of the rolling bearing elements, applied instrumentations, and the test procedures to detect the defect on the bearing will be explained in detail. 


\section{Chapter 3}

\section{Experimental Set up and Instrumentations}

This Chapter introduces the design of the test rig and construction of the test rig installed at the Tribology group of NRC-IAR to carry out experiments on rolling element bearings. This proof of concept design of the test rig allows a range of moderate radial loads to be applied to rotating roller bearings with and without defects in order to establish a correlation for the fault growths on a bearing. This test rig is very useful tool because it has integrated sensors to measure bearing operating conditions and it has the ability to incorporate variety of external sensors that can be used perform bearing health-monitoring based on various fault detection techniques. For the purpose of this study, only sensors that were used to detect bearing defect signatures based on vibration are presented, since the other sensors and techniques dealing with temperature, torque measurements and oil debris monitoring methods are generally unable to provide any information on the kind of the defect and its growth signature. The test rig functionality and the motivation behind the design of the major rig components will also be explained in this chapter.

\subsection{Test Rig Design}

In order to allow the use of test bearings of various sizes, it was decided to use a modular design for the test rig. This type of design was chosen because of its ability to be disassembled without removing slave bearings, thereby allowing the test bearing to be regularly inspected throughout the test program. Furthermore, it allowed efficient assembly of the defective components with minimal disruption to the test rig. 
As shown in Figure 3.1, the test rig comprises four major parts: the frame assembly, test bearing assembly, belt drive assembly and the load application assembly.

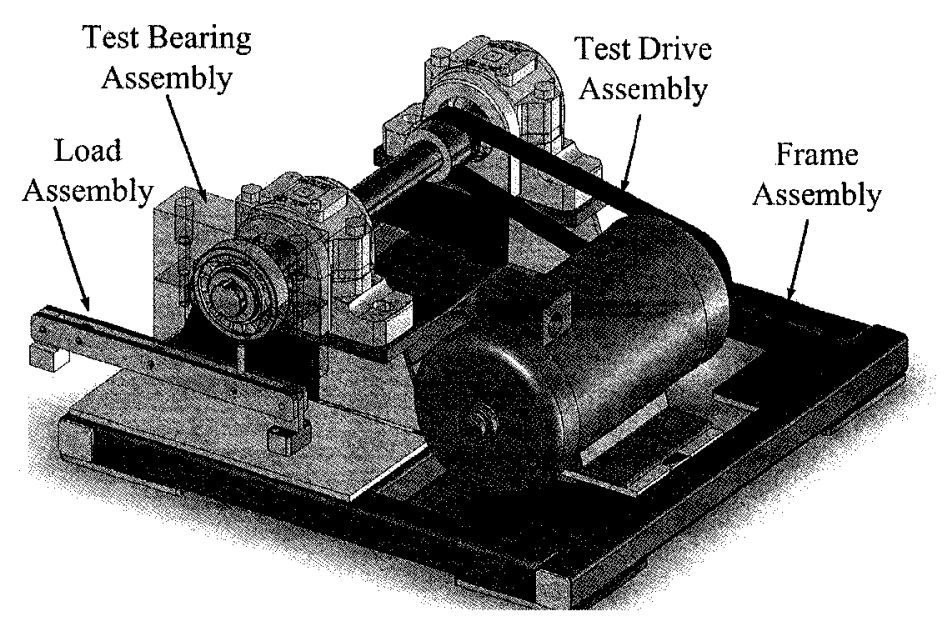

Figure 3.1 Bearing test rig with various assemblies [Tribology group, NRC]

\subsubsection{Frame Assembly}

The frame is a welded assembly consisting wall structural steel tubing. The mount pads are made from cold-rolled steel. After welding the tubes and the mount pads, the bottom surface of the mount pads were machined in order to establish a flat mount plane. This was done to ensure that no unpredicted forces applied to the frame as a result of being fixed to a flat surface exist. The base of the rig was designed in order to allow all components to be securely mounted to avoid undesired vibration. 


\subsubsection{Test Bearing Assembly}

The test bearing assembly has been designed in such a way that it can be switched from one test bearing to another and it facilitates the variation of the shaft speed as well as the applied load.

The primary components of the test bearing assembly shown in Figure 3.2 are composed of the support bearing, test bearing, outer bearing housing and inner race adapter. A deep groove ball bearing was selected as the support bearing because it generates less vibration than other type bearings.

The test bearing used for this study was the normal radial clearance, roller bearing SKF 210EC as shown in Figure 3.3. The test bearing was placed inside a housing, which covers the test outer race bearing. The test bearing basic dimensions shown in Table 3.1 were used to design the outer bearing housing, which was machined from AL 6061T6. The bearing housing was made of Aluminum because of its machinability and cost. The housing inner diameter allows a transitional fit with the bearing outer diameter.

The inner race of the test bearing was free to rotate inside the outer race at shaft speed and outer race was fixed to the housing. The test bearing was cleaned and greased prior to running each test.

Given the bearing geometry, the bearing defect frequencies or characteristic frequencies, were calculated for the chosen test shaft speed in accordance to the equations stated in the section 2.4. The defect frequencies expected from the test are listed in Table 3.2, which includes the outer race ball pass frequency, inner race ball bass frequency, and ball spin frequency related to the shaft frequency. For example, once the shaft is rotating at speed of $900 \mathrm{rpm}$ or $15 \mathrm{~Hz}$, the bearing outer race defect frequency, $f_{b p o r}$ is $101 \mathrm{~Hz}(6.75 \times 15)$. These defect frequencies are applicable in Chapter 5 for the frequency domain analyses. 

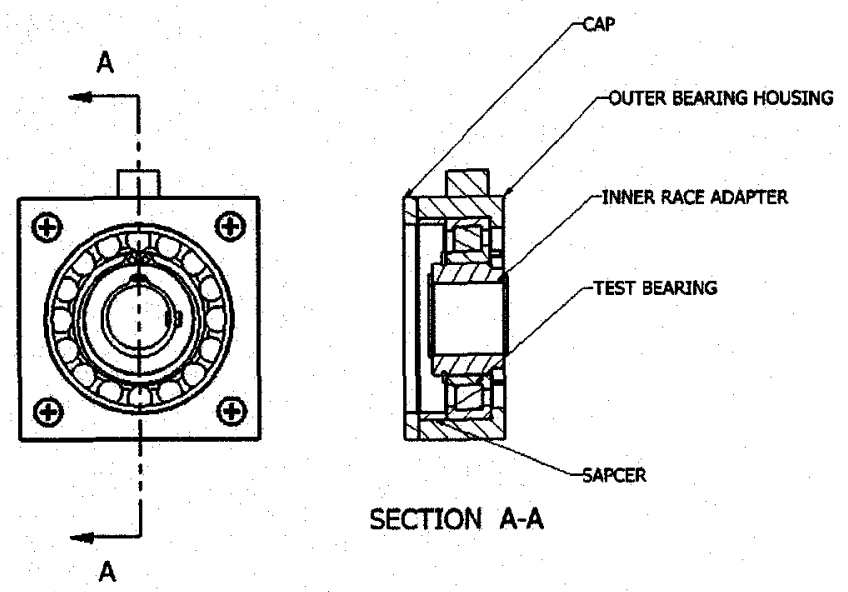

SECTION A-A

Figure 3.2 Test bearing assembly

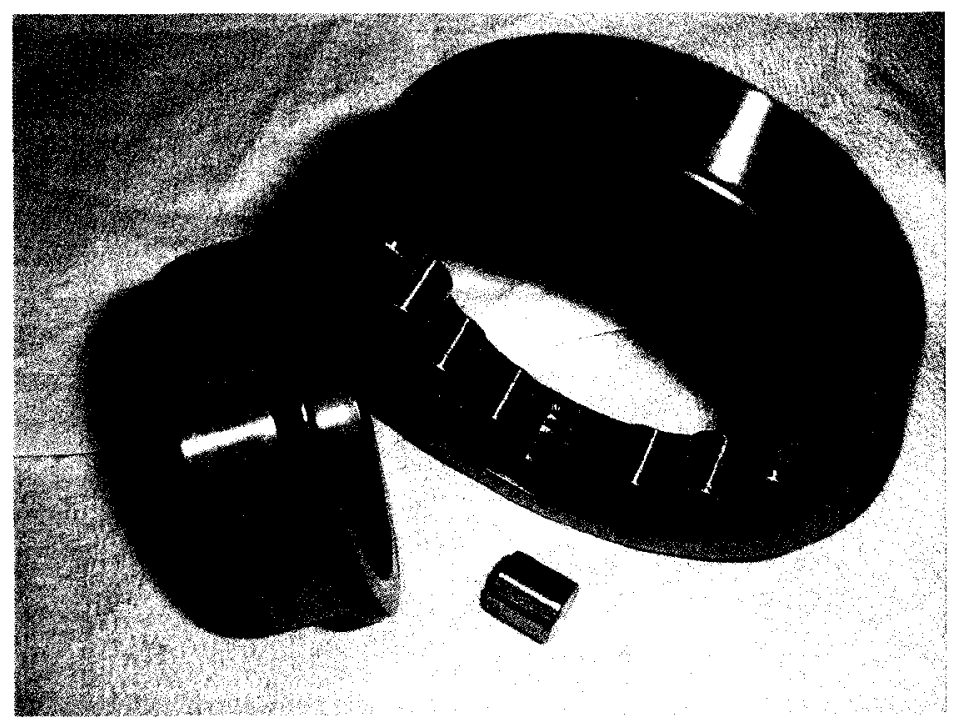

Figure 3.3 Roller bearing SKF 210EC 
Table 3.1 Test bearing dimensions

\begin{tabular}{|c|c|c|c|c|}
\hline Bearing & $\begin{array}{c}\text { Pitch } \\
\text { Diameter, } \\
D(\mathrm{~mm})\end{array}$ & $\begin{array}{c}\text { Roller } \\
\text { Diameter, } \\
d(\mathrm{~mm})\end{array}$ & $\begin{array}{c}\text { Number } \\
\text { of roller, }\end{array}$ & $\begin{array}{c}\text { Angle of } \\
\text { contact, }\end{array}$ \\
\hline SKF 210EC & 70.5 & 11 & 16 & 0 \\
\hline
\end{tabular}

Table 3.2 Defect frequencies

\begin{tabular}{|c|c|c|c|}
\hline Bearing & $f_{\text {bpor }}$ & $f_{\text {bpir }}$ & $f_{\text {bro }}$ \\
\hline $\begin{array}{c}\text { SKF } \\
210 \mathrm{EC}\end{array}$ & $6.75 \times f_{s}$ & $9.25 \times f_{s}$ & $6.25 \times f_{s}$ \\
\hline
\end{tabular}

\subsubsection{Belt drive assembly}

A Direct Current motor provides drive power to the rig and the belt drive assembly ensures the power transmission from the motor to the driveshaft. The motor is able to operate continuously with variable speeds up to $2500 \mathrm{rpm}$. However, only a few constant shaft speeds were selected for the presented set of experimental analysis, namely, 900, 1800 and $2100 \mathrm{rpm}$. The shaft speed is continuously measured using an optical counter. The inner race adapter adapts the inner race of the bearing to the 1-1/4 inch diameter test rig driveshaft, and by keying the inner bore the adapter allows the torque to be transmitted from the rig driveshaft to the inner race of the test bearing. The outer diameter of the adapter is sized for a transitional interference fit with the inner race of the roller bearing. The inner race adapter is a machined component, made from C12L14 low- carbon 
content steel. This material exhibits superior machining characteristics as a result of the addition of lead.

\subsubsection{Load Assembly}

The load application assembly performs two main functions:

- Applying the radial load onto the test bearing,

- Preventing rotation of the bearing housing.

The load application assembly consists of a $2^{\text {nd }}$-class lever assembly with a 5:1 lever ratio with dead weights hung at its free end to vary the applied load. The system was designed to apply loads up to $500 \mathrm{lb}$. The load cell was a substitute component and it was placed at the load application point as opposed to the bearing load point due to spatial constraints. The lever arm was fabricated from cold-rolled bar stock and the load was applied to the test bearing through a pin. All associated components of the load assembly were machined from cold rolled steel (Figure (3.4)). Constant loads of 100, 250, and 500lbf were maintained on the test bearing during the experimental analysis presented in this study.

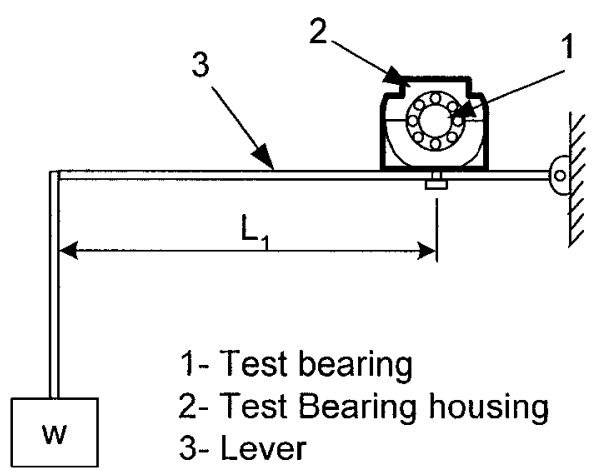

Figure 3.4 The load assembly schematic 


\subsection{Localized Defect Simulation}

Researchers in this field generally use two approaches for creating localized defects on bearings. One approach is to make intentionally the defect on it, measure the response and compare it with healthy bearing response. The other approach is to run the bearing until failure by monitoring the changes using the sensors output (Nishio et al (1979) and Rief et al (1998)). In this type of studies, the bearing failure is generally accelerated though overloading or shock loading of the tested bearing. However, the latter approach is time-consuming and very expensive to implement.

For this study, the Electro Discharge Machining (EDM) technique was employed to create grooves with widths of $0.2,0.5,1.0,1.5$, and $2 \mathrm{~mm}$ on the roller, inner, and outer races separately, simulating damage from fatigue cracks or other related defects (see Chapter 2). Figure 3.5 shows the formed fault using the EDM technique with constant length of $1 \mathrm{~mm}$ on the roller and the races.

For each defect size on each part, this study investigated the effect of variation in the shaft speed and the applied load.

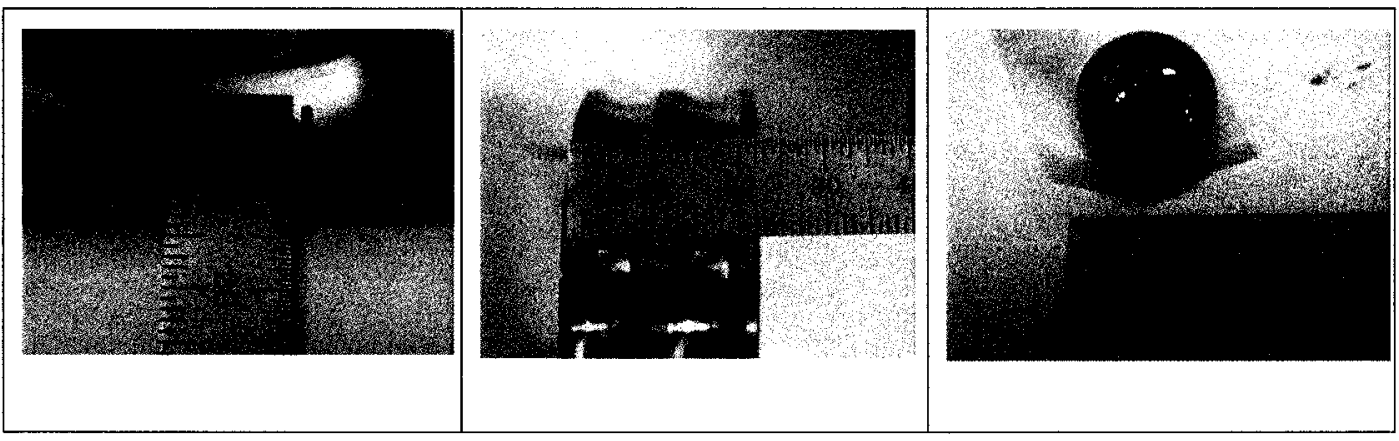

Figure 3.5 Created bearing defects, length of $10 \mathrm{~mm}$ and width of $1 \mathrm{~mm}$ 


\subsection{Instrumentation}

There are a number of available vibration measurement sensors, such as accelerometers, velocity pick-ups, vibrometers, and proximity probes, to name a few. In order to ensure that vibration measurements are accurate several aspects must be satisfied. For instance, the measurement instrument must be calibrated, the frequency and dynamic range of the sensor should be higher than the test system, the sensor mass should not change the dynamic characteristic of the tested object. In order to correlate vibration measurements from several sensors, it is important to insure that they are always collected at the same location and sometimes even at the same instant of time (Howard, 1994).

The existing systems of bearing health monitoring are primarily based on vibration analysis using accelerometers as sensors. The problem encountered with accelerometers is that the signal to noise ratio is poor and it is very difficult to extract the defect signature from noise in practical applications. In addition, accelerometers have to be installed on the bearing or even gearbox casing, which may be far away from the defect source. Moreover, the transmitted signal could be attenuated and distorted due to the intermediate components. In order to identify the capability of sensors other than accelerometers to detect bearing condition, the data was acquired through a set of measurement sensors listed in Table 3.3. They were located at the vertical and horizontal directions of the faulty bearing. The sensors were connected to a high-speed analog-to-digital data acquisition board installed on a portable computer.

A tachometer was installed on the test rig to provide a once per revolution synchronizing pulse, which was used to trigger the start of data capture. This ensures that the signals acquired from all sensors are synchronized with respect to a known shaft position.

A DAQ- 6036 data acquisition card was used for the analog to digital conversion and recording. It captured 6 channels of data continuously to a hard drive at a sample rate of $100 \mathrm{KHz}$ per channel. A dedicated portable computer was set up to 
simultaneously record the vibration data from the test bearing through all the sensors during the operation of the used data acquisition software (National Instruments Labview).

For each simulated defect case, 9 data files were recorded, providing over 2 seconds of data per fault simulation. This was equivalent to shaft revolutions at 900,1800 , and $2100 \mathrm{rpm}$. A total of 61,440 data points were recorded per acquisition (data file) at the sampling rate of $30 \mathrm{KHz}$, chosen based on impact tests (see Section 3.4).

To simulate realistic diagnostic conditions, the timing of data acquisition was randomly selected within a 5-minute test period. It was felt that this approach was representative of the method to be employed at 9 operational conditions tested. Recorded files were then loaded into MATLAB to perform the postanalysis using its signal processing toolbox.

\subsubsection{Accelerometer}

Piezoceramic accelerometers have a microscopic crystal structure that produces a voltage output proportional due to the acceleration forces applied.

The existing methods of the bearing vibration analyses that are generally based on accelerometers have presented good performance. Shiroushi et al (1997) showed that the accelerometer was able to detect defects on a bearing as small as $15.4 \mu \mathrm{m}$.

The accelerometers used in this study were two PCB PIEZOTRONICS Model $352 \mathrm{C} 22$, having sensitivity of $9.75 \mathrm{mV} / \mathrm{g}$ and $8.7 \mathrm{mV} / \mathrm{g}$ for radial and axial installation, respectively. The measurement range of these accelerometers was $\pm 500 \mathrm{~g}$ peak, and a frequency response range was up to $20 \mathrm{kHz}$ with an internal resonant frequency greater than $50 \mathrm{kHz}$. A signal conditioner PCB PIEZOTRONICS model 482A18 with the maximum voltage gain of 1:100 was connected to these accelerometers to amplify the signals prior to the data recorder. During the tests, the accelerometers were bonded on the top of the test bearing 
housing and on the front surface of the housing to measure radial and axial vibration, respectively.

Table 3.3 Instrument conditions

\begin{tabular}{|c|c|c|c|}
\hline Sensor & Characteristic & Measurement & Location \\
\hline Accelerometer & $\begin{array}{l}\text { Piezoelectric } \\
\text { Model: } 352 \mathrm{C} 22 \\
\text { Sensitivity: } 9.75 \mathrm{mV} / \mathrm{g}\end{array}$ & Vibration & $\begin{array}{l}\text { Attached radially on the } \\
\text { bearing housing }\end{array}$ \\
\hline Accelerometer & $\begin{array}{l}\text { Piezoelectric } \\
\text { Model: 352C22 } \\
\text { Sensitivity: } 8.7 \mathrm{mV} / \mathrm{g}\end{array}$ & Vibration & $\begin{array}{l}\text { Attached axially to the } \\
\text { bearing housing }\end{array}$ \\
\hline $\begin{array}{l}\text { Air-Coupled } \\
\text { Ultrasound }\end{array}$ & $\begin{array}{l}\text { Frequency range: } \\
40 \mathrm{kHz}-2.25 \mathrm{MHz}\end{array}$ & Sound & Non-contact mounting \\
\hline Piezo-Ultrasonic & UltraTrack 750 & Sound & $\begin{array}{l}\text { Bolted on the top } \\
\text { housing }\end{array}$ \\
\hline Eddy Current & Pencil eddy current & Displacement & Non-contact mounting \\
\hline Strain gage & Qb10s & Displacement & $\begin{array}{l}\text { Bonded on the bar } \\
\text { attached to the housing }\end{array}$ \\
\hline
\end{tabular}

It was found that the accelerometer located on the top of the housing (radial direction) provided the better results. Therefore, this radial accelerometer was 
used to monitor and compare the accuracy of the other sensors. The outputs of all other sensors were recorded simultaneously for the off-line signal analysis.

\subsubsection{Ultrasonic Testing}

Ultrasonic testing has been practiced for many decades. Rapid development in this instrumentation in the past few years was verified. Ultrasonic testing is based on time-varying vibration in materials, which is generally referred as "acoustics". This methodology converts electrical and mechanical energy back and forth.

Bearings nearing failure generate measurable ultrasonic frequencies well in advance of audible and mechanical signals. Therefore, ultrasonic detection is a useful way to monitor incipient bearing failure.

The most commonly measured Acoustic Emission (AE) parameters for bearing diagnosis are counts and events (Mathews, 1983). Counts correspond to the number of times the amplitude exceeds a preset voltage in a given time and provides a simple number that characterizes the signal. An AE event consists of a group of counts and signifies a transient wave.

Tandon and Nakra (1992) investigated AE counts for an outer race defect using a resonant type transducer. It was concluded that $\mathrm{AE}$ counts increased with increasing load and rotational speed. However, it was observed that AE counts could only be used to detect the defect when the defect was less than $250 \mu \mathrm{m}$ in diameter. The applied loads during the above study ranged from $8 \%$ to $50 \%$ of the bearing static load rating. Choudhury et al (2000) employed AE for bearing defect identification on various sized bearings and rotational speeds ranging from $500 \mathrm{rpm}$ to $1500 \mathrm{rpm}$. It was observed that $\mathrm{AE}$ counts were low for undamaged bearings, based on a threshold level of 1 volt. In addition, it was observed that $\mathrm{AE}$ counts increased with increasing speed for damaged and undamaged bearings whilst an increase in load did not result in any significant changes in $\mathrm{AE}$ counts for both damaged and undamaged bearings. 
Further studies by Shiroushi et al (1997) have used an acoustic sensor as well as an accelerometer to detect roller bearing defects and track their progression. Although the acoustic emission sensor provided very interesting results for defects on the outer race, it was unable to provide any indication on the inner race defects. The adaptive noise cancellation technique used for analysis also helped to isolate the defect signatures and significantly increased the signal to noise ratio.

Moreover, Miettinen et al (2000) employed the acoustic emission technique to monitor the lubricant condition in rolling element bearing and successful applications of $\mathrm{AE}$ to bearing diagnosis for very slow rotational speeds have also been reported.

Although there are several advantages of $\mathrm{AE}$ techniques, the $\mathrm{AE}$ can be affected by material microstructure, geometrical arrangement of free surfaces, loading conditions, and the background noise. Moreover, it has been shown that $\mathrm{AE}$ counts are sensitive to the level and grade of lubricant within the bearing, which adds to the complexity of this measurement for damage detection. For these reasons, the investigation presented in this study intends to validate the use of R.M.S, amplitude, and signal power by using two types of ultrasonic transducers: air coupled ultrasonic and piezoelectric ultrasonic for the bearing defect diagnosis.

\subsubsection{Air Coupled Ultrasonic Transducer}

Air Coupled Ultrasonic Transducer (ACUT) is a non-contact measurement device that has become increasingly common for ultrasonic testing. In this method, air acts as a coupling medium between the ultrasonic transducer and the examined object. Hence, in contrast to contact measurements, no coupling gel or immersion in water or liquid is required to perform measurements using ACUT. Therefore, this device can be an important tool for condition monitoring through nondestructive testing.

This sensor is usually applied for extracting structural phenomenon such as crack 
growth or plastic deformation. It generates elastic wave in the air due to materials under stress at very high frequency range (even higher than $100 \mathrm{kHz}$ ). Both the receiver and the source of ACUT usually operate under high electric fields.

Two measurement techniques could be used with this ACUT: the first technique is the Pulse-Echo technique, where ultrasound is pulsed to the surface to be evaluated and its reflection is then captured. This technique is convenient and very useful for surface damage evaluation. The second technique is to use the sensor as a microphone or an emission sensor to capture the sound emitted from the system for evaluation. This second technique has been studied and analyzed previously (Dadouch et al, 2005). The research showed that defects on a roller bearing inner race were readily detectable with an air-coupled ultrasound sensor without performing any post-processing on the raw signal. The results of this study using an ACUT were very promising compared to the data obtained with an accelerometer.

The air coupled ultrasonic used in this project has been invented and patented at Queen's University (Kingston, Canada) by Schnidel and Hutchins (1994). The air coupled ultrasound transducer was used as a microphone to capture high frequency signals from the damaged bearing and was externally supported by bars. Figure 3.6 shows the test setup and the micro-machined capacitance transducer with a focus plate used to measure ultrasonic signals. This sensor was composed of a micro-machined Fresnel zone-plate as illustrated in Figures 3.6(b) and 3.6(c), which was mounted on a planar, micro-machined, air-coupled capacitance transducer, which was capable of generating tonebursts of ultrasonic waves in air over a wide frequency bandwidth (Schnidel et al 1999). 


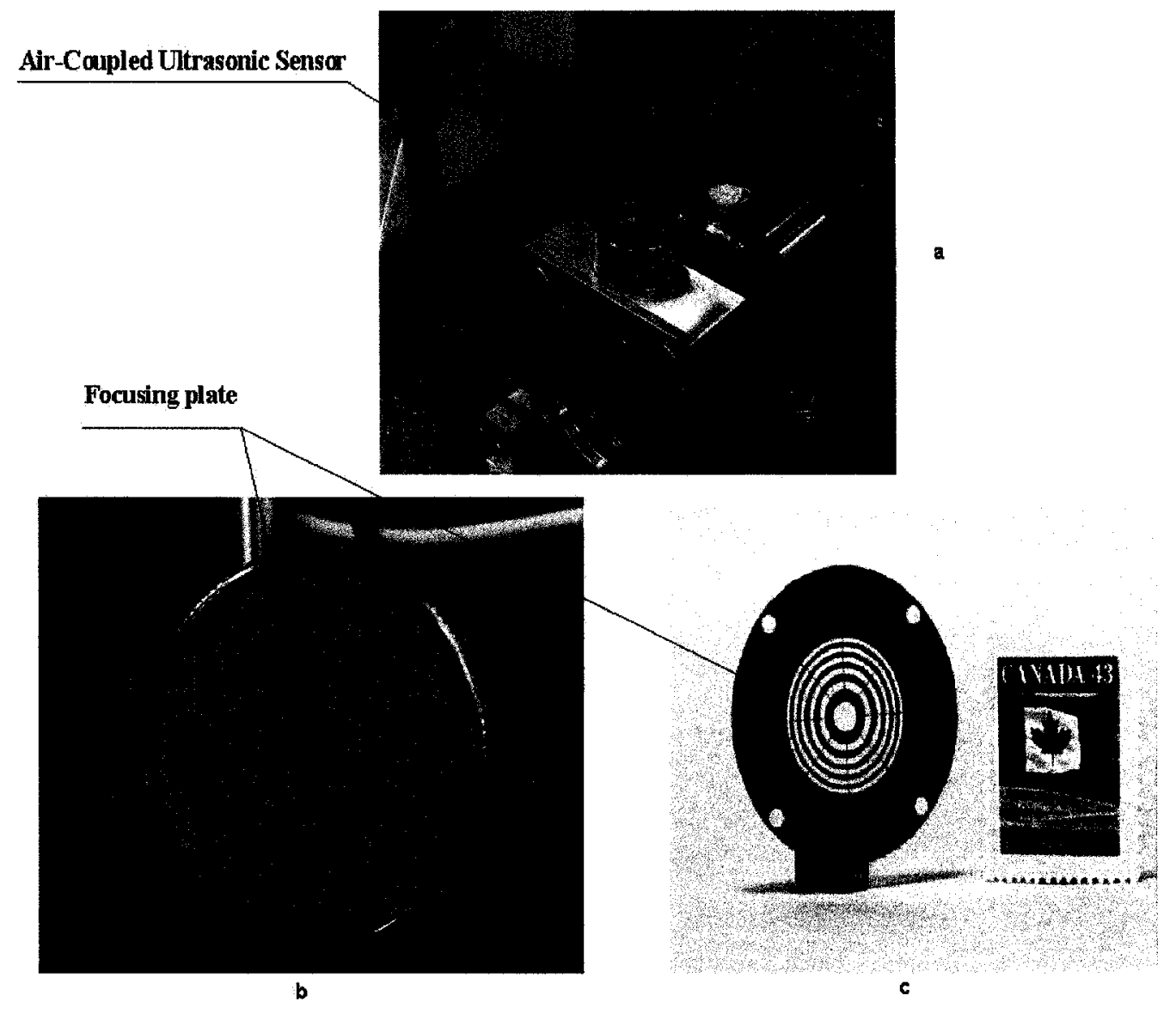

Figure 3.6 (a) ACUT set up for test bearing,(b) and (c) The capacitive ultrasonic Transducer [Tribology group, NRC]

Also, a low noise preamplifier was used to maximize the damage detection performance of the system. The preamplifier, Model SR 560, was applied directly to the receive transducer, so as to minimize any noise pickup on the cables that ranged from 40 to $60 \mathrm{~dB}$. The signal output from the pre-amplifier was connected (i.e. via BNC/coaxial cable) directly to a commercial data acquisition card in the laptop.

In this study, for damaged bearings, whenever the rolling elements rotate across the defect in races or a defect in the rolling element hits the races, the bearing elements are stressed and the acoustic emission was expected to increase considerably. 


\subsubsection{Piezoelectric Ultrasonic Transducer}

The primary element of an ultrasonic sensor is the active element that converts the electrical energy to acoustic energy, and vice versa. The active element of Piezo Ultrasonic Transducer (PUT) generally consists of a piece of polarized piezoelectric material where parts of the molecule structure are positively charged, while other parts are negatively charged by the electrodes attached. By applying an electric field, the polarized molecules align with the electric field, resulting in induced dipoles within the molecular and crystal structure of the material. This alignment of molecules causes the material to change dimensions. This phenomenon is known as electrostriction.

An internal view of the contact PUT is shown in Figure 3.7. The backing material supports the piezoelectric crystal, which has a great influence on the damping characteristics of the transducer. The piezoelectric element is usually cut to $1 / 2$ the desired wavelength. An impedance matching (wear plate) is placed between the active element and the face of the transducer to increase the acquired energy. (Vive, 2004)

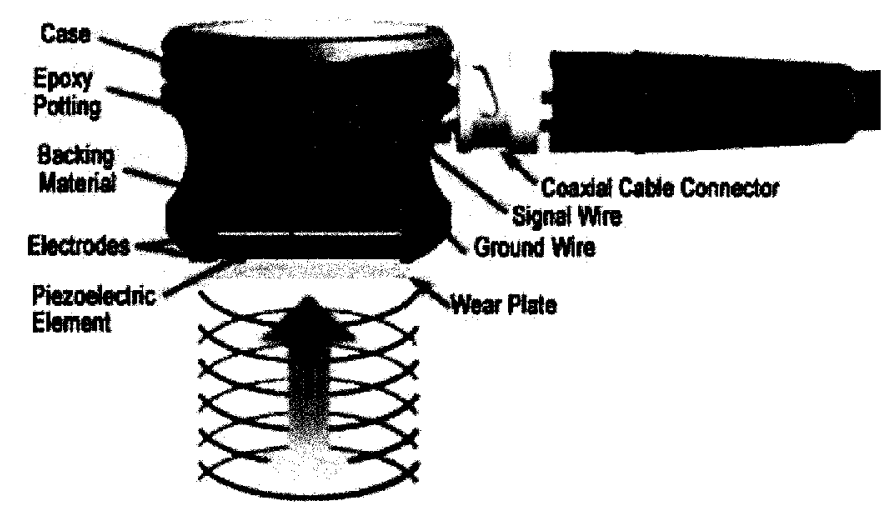

Figure 3.7 Internal View of PUT 
Researchers have investigated the use of this PUT for damage detection on bearings to compare changes in sound pressure and sound intensity levels associated with defective bearing and healthy one.

However, for this study, the output voltage of this sensor was considered the acoustic signal. The applied PUT of the Ultra Track 750 sensor is a contact transducer type referred as "Airborne Ultrasonic". Airborne Ultrasonic is concerned with the transmission of ultrasonic waves through the atmosphere without the need of a sound conductive gel. Since the top of bearing housing is a flat surface with a hole in the middle, the sensor was bolt mounted on the housing to capture the ultrasonic waves in the tests, as illustrated in Figure 3.8.

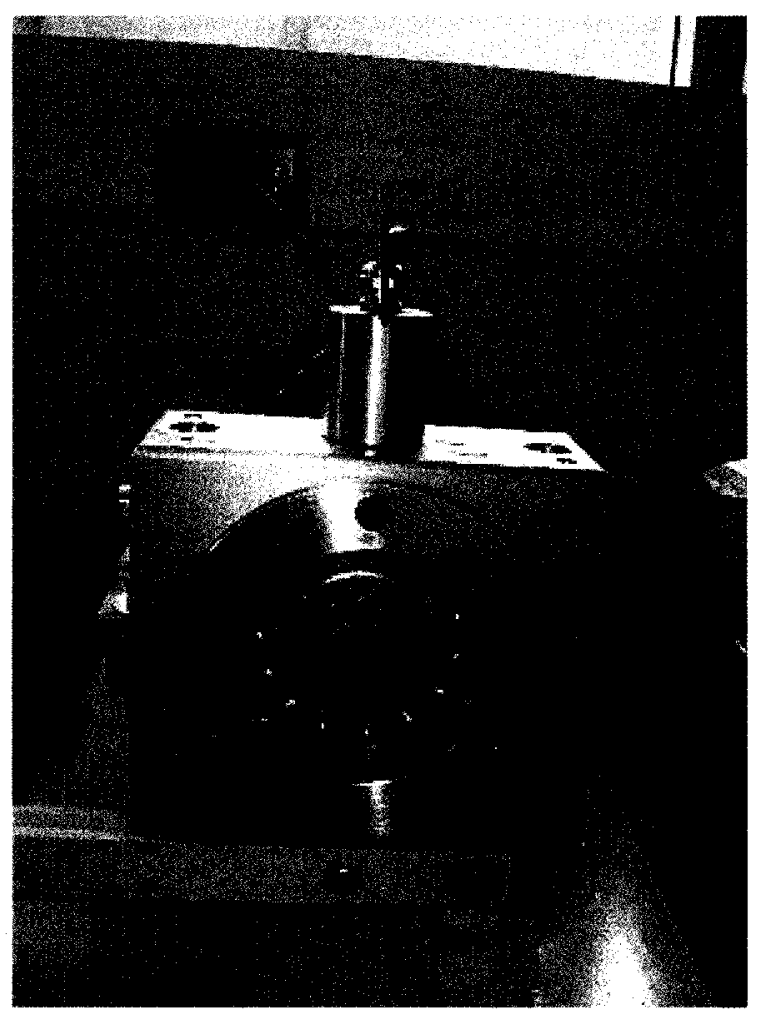

Figure 3.8 Piezoelectric ultrasonic sensor on bearing 


\subsubsection{Eddy Current Sensor}

Eddy current inspection is one of the several NDT methods that use the principles of electromagnetism as the basis for conducting examinations. Eddy current sensors are widely used for non-contact position, displacement, and proximity measurements. Operating on the principle of magnetic induction, these detectors can precisely measure the position of a metallic target. Movement of the target or any change in the characteristics of the target (crack, different material, etc.) is measured as an impedance change by the Eddy current sensor. It can be then measured by an impedance meter or an oscilloscope device.

In the past, Eddy current transducers have been used very effectively in monitoring machines operating with fluid journal and thrust bearings (Mitchell, (1993), Westbrook and Turner (1994)). These transducers allow the measurement of the relative position of the shaft within the bearing as well as its orbit. Several methods are usually available for the installation of eddy current transducers, including internal, internal/external, and external mounting.

There are several types of Eddy current probes; the probe type used for these tests was an absolute probe. Absolute probes generally have a single test coil that is used to generate the eddy currents and sense changes in the eddy current field. The above probe was chosen as the Eddy current instrument to detect the defects on bearings because of its specific characteristics such as sensitivity to small cracks and defects, ability to perform measurement without the need to contact the test subject and its portability.

An Eddy current probe with a Zetec MIZ 22 was used to perform the eddy current measurement. The data was sampled at $100 \mathrm{kHz}$. The transducer was positioned on top of the housing to measure its vertical displacement and was externally supported as shown in Figure 3.9. The instrument was set up in a manner where the lift-off response had only a vertical displacement. Since this probe was a conventional "pencil". Eddy current probe has been designed as a contact probe for crack detection with very limited range for lift-off. It also needed to verify the 
measuring range (maximum probe-to-metal spacing) and linearity between lift-off variation and output values. In this experiment, the probe was approximately $1 \mathrm{~mm}$ above the housing. A simple experiment showed that with the parameters used during bearing vibration measurement, the measuring range for this probe was $1 \mathrm{~mm}$ (Dadouch et al, 2005). Therefore, the distance of probe and bearing housing was kept in the range of $1 \mathrm{~mm}$ to maintain the linear range of the Eddy current probe and the generated signal was proportional to the induced vibration

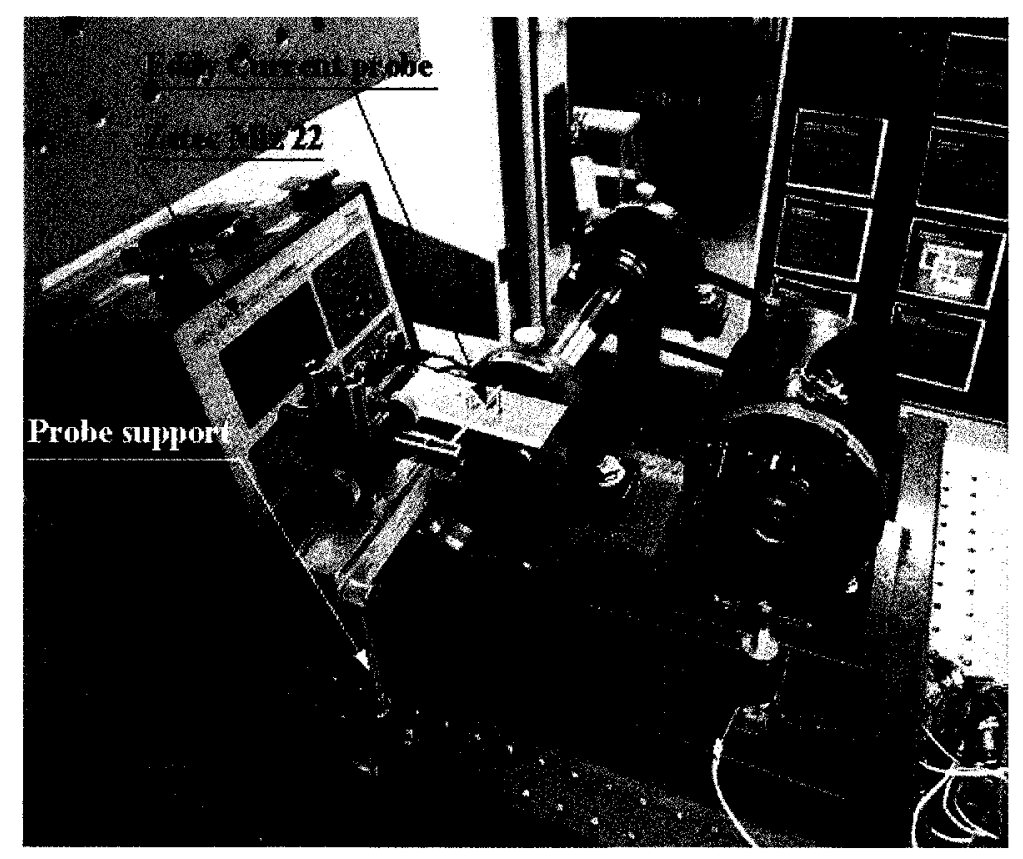

Figure 3.9 Eddy current probe and Zetek unit

\subsubsection{Piezoelectric Strain Gage}

Piezoelectric elements are commonly used in smart structural systems as both sensors and actuators (Chopra, 1996). A key characteristic of these materials is the utilization of the converse piezoelectric effect to actuate the structure in addition to the direct effect of sensing structural deformation. 
The capability of a piezoelectric element, as strain sensor for the detection of defect on a bearing was investigated. Strain was measured in terms of the change in the electric charge generated by the element as a result of the direct piezoelectric effect.

\subsubsection{Beam Characteristic}

The Piezoelectric sensor was surface bonded to a beam that was attached to the bearing housing at one end. The other end was attached to a vertical post as shown in Figure 3.10.

In order to be able to recognize the characteristic frequency (Section 3.3) of a defective bearing system through signal processing, a beam with fixed dimensions $(6 \times 0.6 \times 0.03$ inch) was chosen such that the first three resonant frequencies (Table 3.4) were at least $\pm 30 \mathrm{~Hz}$ of the calculated bearing defect frequencies (Table 3.2).

Table 3.4 Beam resonant frequencies

\begin{tabular}{|c|c|c|c|}
\hline \multirow{2}{*}{$\begin{array}{c}\text { Bending mode } \\
\text { (Natural frequency) }\end{array}$} & $f_{1}$ & $f_{2}$ & $f_{3}$ \\
\cline { 2 - 4 }$(\mathrm{Hz})$ & 168.3 & 463.9 & 909.4 \\
\hline
\end{tabular}




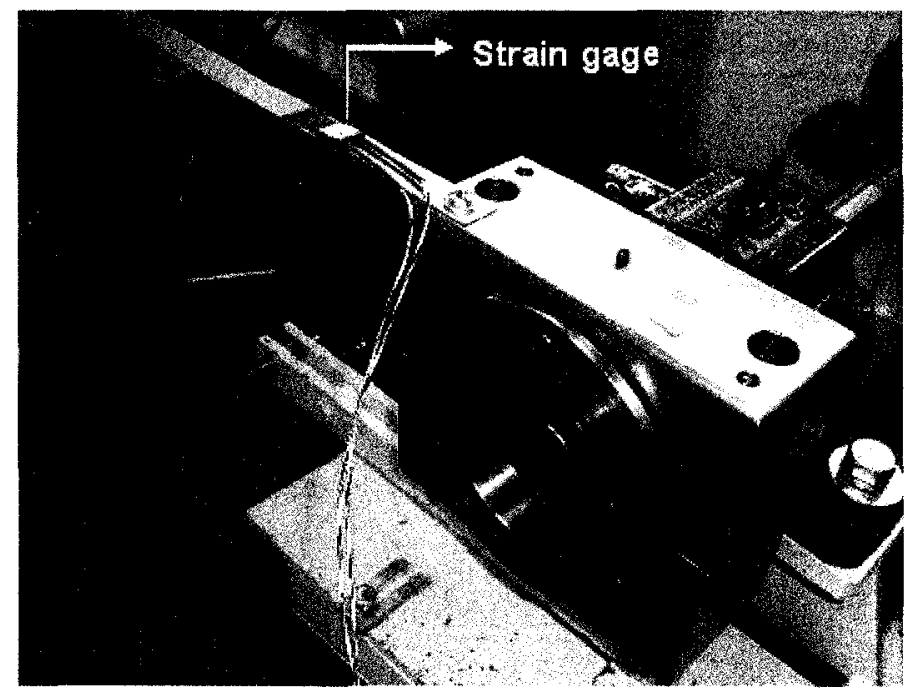

Figure 3.10 Piezoelectric strain gage on the beam attached to the bearing

\subsection{Sampling Frequency Determination}

To record accurate samples of data per second through the instruments, it was necessary to select the optimum sampling frequency. The frequency limitation on the use of a sampling system is based on the Sampling Theory. It states that the used sampling frequency must be at least twice the highest frequency expected from the measurement system to avoid the aliasing phenomenon.

The "envelope analysis", one of the applied techniques on the vibration signals, makes use of the resonant frequencies of the bearing physical system, which usually occur at higher frequency ranges. To be able to determine resonant frequencies from the extracted signals prior to the test, a series of impact tests were performed on the roller bearing to identify vibration modal frequencies that produced the highest amplitude in the dynamic response. An analogue low-pass 'anti-aliasing' filter was used prior to sampling to make sure that there was no frequency above the half the sampling rate to avoid aliasing. 


\subsubsection{Impact Tests}

A series of laboratory impact tests on the roller bearing components (SKF 210 EC) at NRC acoustic lab were devised to determine the appropriate sampling frequency and also to verify the natural frequencies observed while collecting vibration signal.

\subsubsection{Test Fixture}

The bearing subjected to the impact tests was clamped between two surfaces under a compressive force. Radial impacts on inner and outer rings were applied while accelerometers located on these components were used for dynamic response monitoring. This test fixture is shown in Figure 3.11.

The vibration signal levels from the accelerometers during these impact tests of the bearing were recorded through a LMS data acquisition system connected to a PC. The metallic hammer head was selected so that the excitation frequency was within the range up to $20 \mathrm{kHz}$.

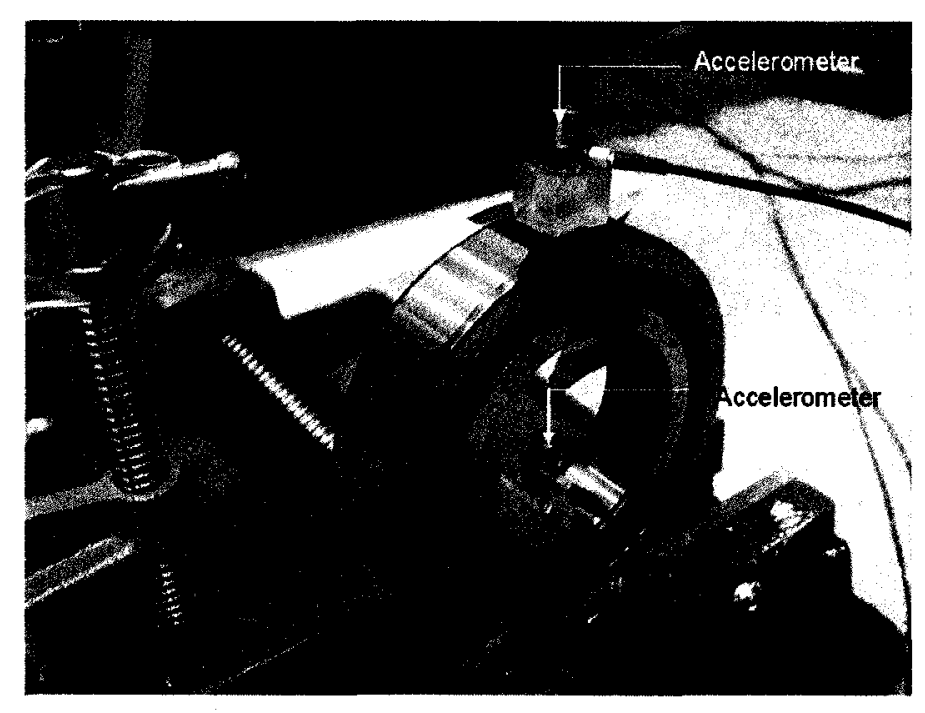

Figure 3.11 Test fixture for impact test on the roller bearing 


\subsubsection{Impact Test Results}

The intent of this test was to identify the resonant frequencies inherent to the bearing to determine the adequate sampling frequency for the series of vibration tests.

The results of these resonance tests are shown in Figure 3.12 and 3.13 as Frequency Response Function (FRF) plots of frequency versus amplitude. Several resonances were picked up from the bearing system. It is observed in these Figures that the first resonance mode of the bearing occurred at $1.735 \mathrm{kHz}$ and the second mode at around $3.873 \mathrm{kHz}$ and that the highest resonant frequency associated with high amplitude vibration mode was approximately at $17 \mathrm{kHz}$.

Frequencies higher than $18 \mathrm{kHz}$ represent were related to the accelerometer natural frequency, which is around $20 \mathrm{kHz}$ according to its specification sheet.

The above results showed that all of high amplitude vibration modes were located below $17 \mathrm{kHz}$. Based on these data, a sampling frequency of $30 \mathrm{kHz}$ was set for all tests. These resonant frequencies were obviously related to the mass of the components and the stiffness of the supporting contact between the roller and races.

It should be noted that, the frequency of the first mode of the inner race was around $1.7 \mathrm{kHz}$, the second modal frequency at $3.8 \mathrm{kHz}$. In the high frequency range, there was high modal response around $15 \mathrm{kHz}$ 


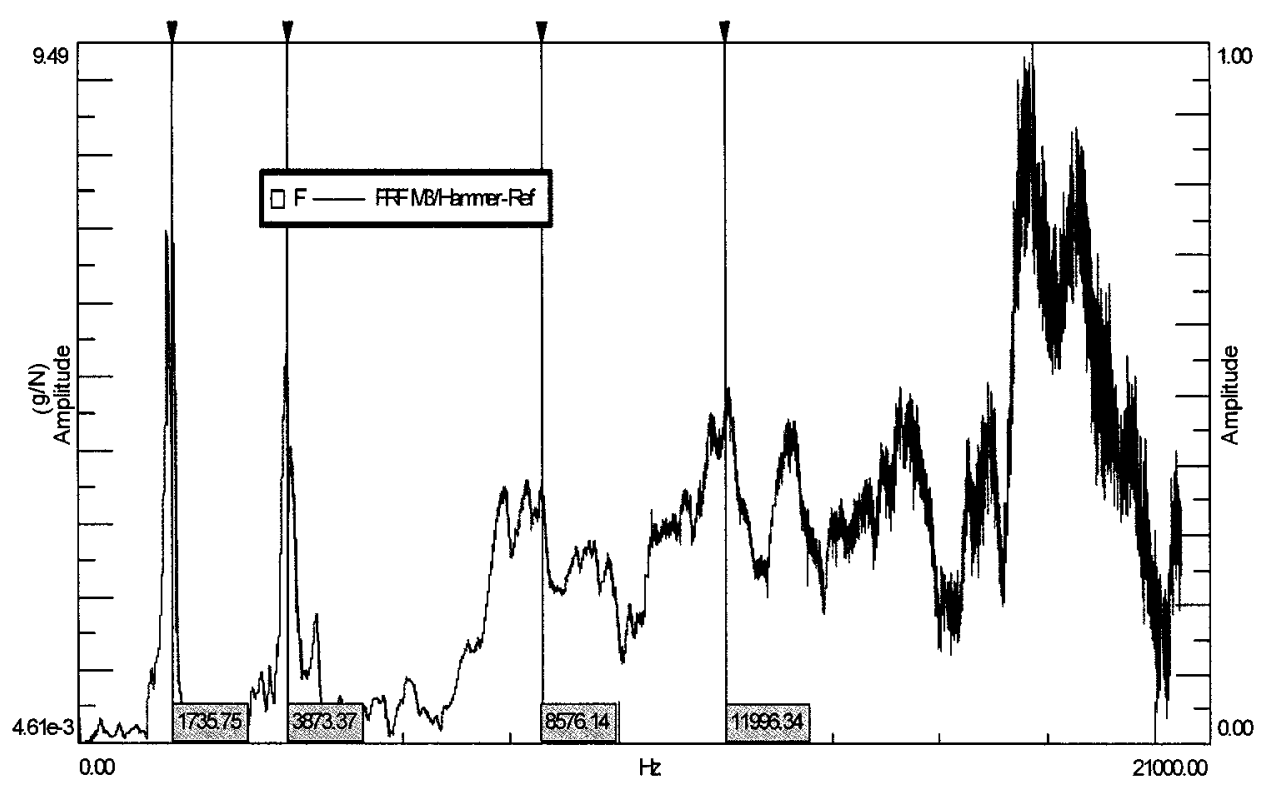

Figure 3.12 FRF of the rolling bearing, Accelerometer on the outer race

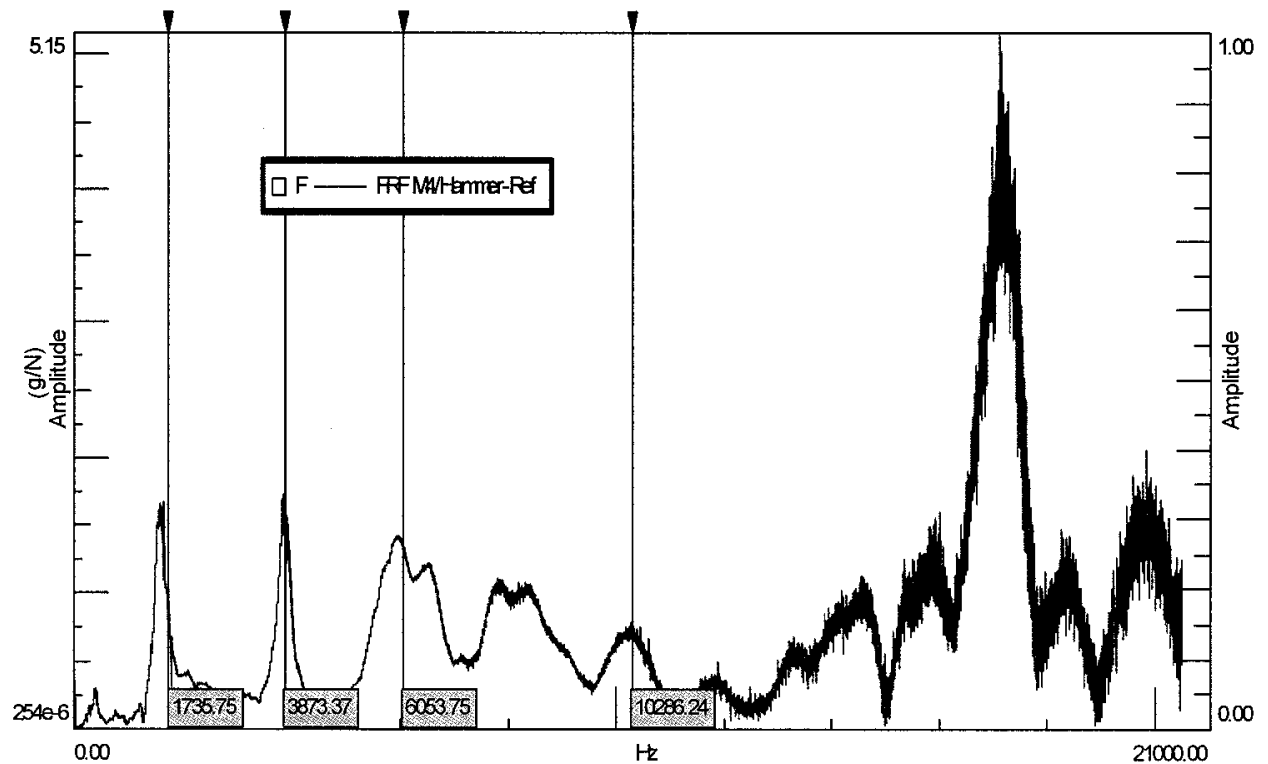

Figure 3.13 FRF of the roller bearing, Accelerometer on the inner race It could be concluded, cautiously, that one or more of these resonant frequencies would be excited by localized defects generated in a defective bearing. 
The conclusions from the impact tests are summarized as follows:

- The inner race of the test bearing exhibited several resonant frequencies in the range of 1.8 to $17 \mathrm{kHz}$.

- The resonant frequency of the inner race was transmitted through the housing to the sensing accelerometer during the tests.

- The outer race of the test bearing also showed resonant frequencies in the range of 1.8 to $17 \mathrm{kHz}$, too.

\subsection{Vibration and Acoustic Test Procedures}

The test procedure followed when conducting the tests on the roller bearing with or without a defect was as follows:

i. Mount the sensors. The accelerometers were bounded at two locations on the bearing housing. One was placed in the radial direction and the other was installed in the axial direction of the housing. The piezo ultrasonic sensor was bolted on the bearing housing. The Eddy current probe was positioned on top of the housing by an external support and maintained the maximum probe-to-housing spacing of $1 \mathrm{~mm}$. The air coupled ultrasonic sensor was placed near the top of the housing at a maximum distance of 4 in. It was important to keep the top surface of the bearing housing mounting surface clean and paint-free. Any irregularities in the mounting surface preparation might be translated into improper measurements and erroneously detected as damage by the sensors.

ii. Lubricate the each component of test bearing using the grease.

iii. Apply the radial load to the predetermined weight by load assembly system. 
iv. Turn on the motor and adjust the gain to achieve the desired speed.

v. Wait for warm-up and steady operation.

Note: The bearings with the outer race defect were mounted on the test rig in such a way that the defect was located upwards in the zone of maximum load.

vi. Connect all the sensors to the data acquisition system using BNC connectors.

vii. Collect the data simultaneously through the data acquisition software, the National Instruments Labview software.

viii. Retrieve the data in MATLAB environment for post-processing and analyze the signals according to the chosen technique.

\subsection{Summary}

The test rig, applied sensors, fault generation purpose, operation conditions, and data recording system to conduct series of experiments on rolling element bearings have been described in this Chapter.

The impact test performed in order to choose the adequate sampling frequency has been described. These results will be compared with the impact tests due to fault on the bearing in Chapter 5 . 


\section{Chapter4}

\section{Vibration Analysis Techniques on Rolling Elements}

In Chapter three the experimental bearing test rig, the applied sensors on the test bearing and associated measurement variables were described. This Chapter provides a review of available signal processing techniques used for fault detection and condition monitoring in rolling elements by highlighting the advantages and disadvantages of each technique. A summary of the findings is then compiled to justify which techniques should be investigated further.

\subsection{Time-Domain Analysis}

Time-domain metrics play a critical role in analysis of simple machine components but are not viable for complex components. These time-domain analyses may detect the failure of these components allowing replacement prior to total failure. Although the damaged component may be beyond repair by this time, the component replacement cost is generally insignificant compared to the potential cost of catastrophic failure of the machine. Dyer and Stewart (1978) discovered that the probability density of the acceleration signal of a bearing in the perfect condition has a Gaussian distribution, whereas a damaged bearing results in a non-Gaussian distribution with domain tails because of an increase in the number of high levels of vibration components. Generally used time-domain metrics include waveform analysis and vibration features. 


\subsubsection{Waveform Analysis}

In the early days of vibration analysis, time waveform data was viewed in oscilloscopes while frequency components were calculated by hand. This type of analysis could determine the presence of the defect in the vibration signature. However, the drawback of waveform analysis on defect detection is the need for manual calculation of the repetition frequency based on the time difference observed between feature points and the need for an expert who controls the oscilloscope and clarifies the results.

\subsubsection{Overall Vibration Level}

The vibration signal features are trended against time as an indicator of failing machine condition and/or compared with published vibration criteria. A number of simple signal metrics based on time domain analysis still have some application in mechanical fault detection and the simplest of these are the peak and the RMS value of the signal that are being used for the overall vibration measurement.

\subsubsection{Peak Value}

The peak level of the signal is defined simply as half the difference between the maximum and minimum vibration level:

$$
\operatorname{peak}=\frac{1}{2}(\max (x(t))-\min (x(t)))
$$

Because the peak level is not a statistical value, it is often not a reliable indicator of damage; false data caused by noise may have affects on the peak value. Therefore, the RMS value is generally preferred to the peak level in machine condition monitoring. 


\subsubsection{RMS Value}

The RMS (Root Mean Square) value of the signal is the normalized second statistical moment of the signal. In other words, it is the standard deviation of the signal.

$$
R M S=\sqrt{\frac{1}{T} \int_{0}^{T}(x(t)-\bar{x})^{2}} d t
$$

where $\mathrm{T}$ is the length of operating time and $\bar{x}$ is the mean value of the signal.

For discrete (sampled) signals, the RMS of the signal is defined as:

$$
\begin{aligned}
& R M S=\sqrt{\frac{1}{N} \sum_{n=0}^{N-1}(x(n)-\bar{x})^{2}} \\
& \bar{x}=\frac{1}{N} \sum_{n=0}^{N-1} x(n)
\end{aligned}
$$

The RMS of the signal is commonly used to describe the 'steady-state' or 'continuous' amplitude of a time varying signal.

\subsubsection{Waveform Features}

With a higher number of fault types, the shape of the signal is a better indicator of damage than the overall vibration level. In other words, trending of the wave shape metrics can also be used to help identify deteriorating condition. Bearing faults, which produce short term impulses may not significantly show the overall vibration level but may cause a statistically significant change in the shape of the 
signal.

Crest factor and kurtosis are often used as non-dimensional measures of the shape of the signal waveform. Both these signal metrics increase in value as the signal level increases.

\subsubsection{Crest Factor}

The crest factor is defined as the ratio of the peak value to the RMS of the signal:

$$
\text { Crestfactor }=\frac{\text { Peak }}{\text { RMS }}
$$

The crest factor is often used as a measure of the impulsive nature of a signal. It will increase in the presence of discrete impulses, which are larger in amplitude than the background signal but do not occur frequently enough to significantly increase the RMS level of the signal. It is important to note that the value of the crest factor varies in the presence of random noise.

\subsubsection{Kurtosis}

Kurtosis is the normalized fourth statistical moment of the signal. For continuous time signals this is defined as:

$$
\text { Kurtosis }=\frac{\frac{1}{T} \int_{0}^{T}(x(t)-\bar{x})^{4} d t}{(R M S)^{4}}
$$


For discrete signals the kurtosis is:

$$
\text { Kurtosis }=\frac{\frac{l}{N} \sum_{n=0}^{N-1}(x(n)-\bar{x})^{4}}{(R M S)^{4}}
$$

Kurtosis provides a measure of the impulsive nature of the signal. Increasing signal to the fourth power effectively amplifies isolated peaks in the signal.

Some evaluation of the nature of a signal can be made without trend information because of the non-dimensional nature of the crest factor. For example, both waveform metrics give a value of 0.0 for a DC gain and 1.0 for a square wave. For a sine wave, the crest factor will be $\sqrt{2}$ and the kurtosis will be 1.5 . For normally distributed random noise, the kurtosis will be 3.0 and the crest factor will be approximately 3.0 .

Faults which produce a small number of isolated peaks, similar to ones found at the initial stage of bearing damage, may cause an increase in the crest factor and kurtosis, but as the damage becomes more widely spread, a large number of impulses may occur causing both the crest factor and kurtosis to decrease again. Both the kurtosis and crest factor will decrease if the numbers of pulses increase resulting from the increased the RMS value of the signal without an increase in the individual pulse height. The wave shape features will not identify faults unless the amplitude of the vibration from the faulty component is large enough to cause a significant change in the total vibration signal. This limits their use to components whose vibration signature forms a significant part of the measured overall vibration.

Tandon et al (1992) examined the detectability of each time parameter with the acceleration sensor on defective bearings and compared them with acoustic 
measurement techniques. He showed that statistical parameters such as crest factor, kurtosis, and impulse factor are sufficiently robust to detect fault, under varying bearing operating conditions.

\subsection{Frequency Analysis}

Frequency analysis is a method used to extract the frequency content of the timedomain signal. Spectrum analysis is the most common technique for health management in rolling elements, and it is an important tool for detection and fault diagnosis in simple rotating machinery. Frequency analysis is a measure of the vibrations over a large number of discrete neighboring narrow frequency bands.

For bearings operating at an identified constant speed and geometry, the frequencies of the vibrations produced by the various bearing components can be estimated as described in Chapter 2.

Often, the fault detection using the overall vibration level and/or wave shape features can be significantly improve by dividing the vibration signal in to a number of frequency bands prior to analysis. A fault may not cause a significant change in the overall vibration signal and this may be masked by higher energy generated by non-fault related vibrations. However, the defect may produce a significant change in a band of frequencies in which the non-fault related vibrations are sufficiently small.

\subsubsection{Uncertainty Principle}

All frequency analysis is subject to an uncertainty principle due to the analogous in quantum mechanics, expressed by Werner Heisenberg in 1927.

Frequency analysis made with bandwidth of $B$ hertz for each measurement and duration in time of $T$ seconds has a bandwidth-time limitation of:

$$
B T>1
$$


A minimum resolvable frequency for an event with $T$ second duration is $1 / T$ hertz. If an analyzing filter with a bandwidth of $B$ hertz is used, at least $1 / B$ seconds will be required for a measurement.

The measurement uncertainty due to the bandwidth-time limitation requires a resolution restriction on the frequency transfer. To resolve frequencies separated by $B$ hertz at least $1 / B$ seconds of data must be taken.

\subsubsection{Spectral Analysis}

The changes in vibration level within a particular frequency band can be generally related to a particular frequency generated by a bearing component. Sometimes, comparing the vibration levels at a specific frequency bands can reveal a fault in the bearing system, which provides some level of diagnosis. Su and Lin (1992) provided details of the spectral differences related to various bearing faults.

Because of the availability of commercial frequency analyzers like the Fast Fourier Transform (FFT) analyzer in the industries, the frequency technique has extensive application for bearing fault detection. However, spectral analysis is still too complicated to be used to interpret the localized fault signatures even in simple machineries.

Randall (2000) and McFadden (1989) described that localized defects may not detected because the short term impulsive vibration produced would convert to a large number of low amplitude lines in the frequency spectrum.

The original procedure used in the spectral technique is the conversion of a timedomain vibration signal into a frequency-domain version. The Fourier Transform of a real time signal can describe in the frequency-domain representation of a signal as shown next: 


$$
x(f)=\int_{-\infty}^{+\infty} x(t) e^{-\mathrm{j} 2 \pi \mathrm{ft}} d t
$$

The inverse process can be applied to convert from a frequency-domain signal to the time-domain:

$$
x(t)=\int_{-\infty}^{+\infty} x(f) e^{j 2 \pi f t} d f
$$

The conversion from time-domain to frequency one can be achieved by the use of narrow band filters or, using the Discrete Fourier Transform (DFT) of digitized data. The vibration level at each frequency represents the vibration over a narrow frequency band centered at the designed frequency, with a bandwidth determined by the conversion parameters employed (Oppenheim, 1989).

The DFT is defined as

$$
x(m)=\frac{1}{N} \int_{n=0}^{N-1} x(n) e^{-j 2 \pi \frac{m n}{N}}
$$

and the Inverse DFT is:

$$
x(n)=\sum_{m=0}^{N-1} x(m) e^{2 \pi \frac{m n}{N} j}
$$

The MATLAB software with Digital Signal Processing Toolbox was used to 
perform the frequency-domain data analysis during this study. The frequency range covered by FFT analysis depended on the selected number of data points of the acquired signal as well as on the sampling rate. The frequency resolution of the FFT is dependent on the sampling rate and the data block size used for the analysis.

\subsubsection{Cepstrum Analysis}

Real cepstrum is the inverse Fourier Transform of the logarithm of the magnitude of the Fourier transform. (Equation (4-13))

$$
C_{x}(n)=\frac{1}{2 \pi} \int_{-\pi}^{\pi} \log \left|x\left(e^{j \omega}\right)\right| e^{j \omega n} d \omega
$$

The complex cepstrum refers to the use of the complex logarithm, not the sequence and the complex cepstrum of a real sequence is also a real sequence.

The cepstrum analysis converts a spectrum back to the time-domain, which has peaks corresponding to the period of the frequency spacing common in the spectrum. These peaks can be used to find the bearing peaks in the original spectra. In this respect, it has advantage over spectral analysis for the fault detection on the bearing. However, cepstrum analysis has been proven as a good tool for faults caused by wear on the bearings, it does not provide any detection for other failure types.

\subsubsection{Power Spectral Analysis}

Although FFT analysis has long been popular to characterize mechanical and electrical systems, the power spectral density (PSD) analysis derived from FFT is especially popular analysis technique for vibration monitoring, fault detection, hidden periodicity finding, and biomedical diagnosis. 
The PSD generally indicates the power of the signal at a given frequency. The spectrum of a discrete time series $P(f)$ is the counterpart of a covariance in frequency domain (Equation (4-14)). That is the Fourier Transform of the covariance function $\gamma(n)$ and vice- versa (Oppenhiem, 1989).

$$
P(f)=\frac{1}{f_{s}} \sum_{n=-\infty}^{+\infty} \gamma(n) e^{-2 j f n / f_{s}}
$$

The covariance function can be derived from the PSD by use of the inverse discrete-time Fourier Transform:

$$
\gamma(n)=\int_{f=-f_{s} / 2}^{f_{s / 2}} P(f) e^{2 j f n / f_{s}} d f
$$

There are numbers of method such as periodogram, multi-taper method, and Welch's method to estimate the PSD of signals. For this study, Welch's method (Stoica, 1997) was chosen to estimate the PSD of the signals at each frequency using a data block of 4028 , overlap of $67 \%$, and a Hanning windowing function.

\subsubsection{Envelope analysis}

Fundamentally, the envelope analysis is based on the concept that an impulse of vibration is generated each time a localised defect in a rolling element bearing makes contact under load with another surface in the bearing. A detailed description of this method is given by McFadden and Smith (1987). The impulse has very short duration compared to the intervals between impulses and its energy 
is distributed across a very wide frequency range. As a result, various resonances of the bearing and the surrounding structure are excited by these impacts. Such impulsive excitation in a bearing is normally repetitive. Therefore, the energy is concentrated in a narrow band, which makes it much easier to detect than that of the dispersed energy. This concept led the researchers to use the High Frequency Resonance Technique (HFRT) also known as envelope analysis. In order to achieve good results with this enveloping technique, it requires selecting a frequency band in which the impulsive components excited by the defect are dominant.

As mentioned in Section 2.4, for a constant rotational speed, the frequency of the pulse generation termed as "characteristic defect frequency" can be determined uniquely by the location of the defect.

It is usual to consider the resonance as being amplitude modulated at the characteristic defect frequency, which makes it possible to detect the presence of a defect and diagnose its location.

The HFRT involves following three steps.

i. Band-pass or high-pass a measured signal with a chosen resonant frequency.

ii. Demodulate the filtered signal with a full-wave rectifier.

iii. Low-pass filter to cancel high-frequency components and retain the lowfrequency information associated with bearing defect.

By using such a high-passed/band-passed filter centered at structural resonances, defect-induced vibration can be separated from the background noise. The subsequent demodulation operation will extract the corresponding envelop of the defect-induced vibration signal. Finally, by transferring the signal to frequency domain the repetitive occurrence of defect/characteristic frequency can be clearly identified. 
The analytical examination of the envelope detection can be described by considering the vibration of a defective bearing, after passing through high pass filter. Then, the vibration at some ringing frequency has the form of a high frequency carrier (the system natural frequency) and a low frequency modulation (the defect frequency, each time a defect is encountered):

$$
x=A_{1}\left(1+A_{2} \cos \left(\omega_{1} t\right)\right) \cos \left(\omega_{2} t\right)
$$

where $A_{1}$ and $A_{2}$ are constants, $\omega_{1}$ is the defect frequency of the system and $\omega_{2}$ is the natural frequency of the system. Once the signal is passed through a rectifier, the squared signal would be:

$$
x^{2}=A_{I}^{2}\left(1+A_{2}^{2} \cos ^{2}\left(\omega_{1} t\right)+2 A_{2} \cos \left(\omega_{1} t\right)\right) \cos ^{2}\left(\omega_{2} t\right)
$$

or, after expanding more and using simple algebra:

$$
\begin{aligned}
\frac{x^{2}}{A_{1}^{2}}= & A_{2} \cos \left(\omega_{1} t\right)-\frac{A_{2}^{2}}{4} \cos \left(2 \omega_{1} t\right)+\frac{2 A_{2}+1}{4} \cos \left(2 \omega_{2} t\right) \\
& \left.-\frac{A_{2}}{2} \cos \left(\left(2 \omega_{2}-\omega_{1}\right) t\right)-\frac{A_{2}}{2} \cos \left(\left(2 \omega_{2}+\omega_{1}\right) t\right)-\frac{A_{2}^{2}}{8} \cos \left(2 \omega_{1}-2 \omega_{2}\right) t\right) \\
& \left.-\frac{A_{2}^{2}}{8} \cos \left(2 \omega_{1}+2 \omega_{2}\right) t\right)+\frac{2 A_{2}+1}{4}
\end{aligned}
$$

In equation (4-18), the defect frequency $\omega_{1}$ appears as a distinct frequency component with amplitude of $A_{2}$. Spectrum of the squared signal shows the exact 
frequency $\omega_{l}$ of the modulation, which identifies which defect is present. Usually this squared signal is filtered to remove high- frequency components, leaving a low- frequency signal resembling the envelope of the original signal.

\subsection{Time-Frequency Analysis}

Signals with time-varying frequency content cannot be treated with the traditional Fourier Transform because this method averages the time varying signal and loses the non-stationary characteristics, which may be important. In order to treat such signals and to provide a time-frequency picture of a signal, the tendency is to use methods to show how the frequency content of the signal changes with time.

Time-frequency techniques map the one-dimensional signal to two-dimensional function of time and frequency. Thus, these techniques present a valid tool to analyze a non-stationary signal rather than using the Fourier transforms. However, each of these techniques has some drawbacks. It also seems that eliminating one of these shortcoming leads to the loss of advantage in another aspect of the analyses.

\subsubsection{Short Time Frequency Transform}

The Short Time Frequency Transform (STFT) and its energy density spectrum has been the most widely used tool for bearing defect diagnosis (Howard, 1994). STFT splits the signal into a set of data sub bands with a fixed window width along the time axis and then performs the Fourier transform on each sub band. To achieve this, at a certain time $\tau$ a sliding window function $h(t)$ applies to the signal $x(t)$ and then the Fourier Transform of the windowed signal is calculated. This generates the STFT of the signal at time t. Mathematically, STFT can be defined as:

$$
S(\tau, f)=\int x(t) h(t-\tau) e^{-j 2 \pi f t} d t
$$


and the energy density spectrum is as follows:

$$
E(\tau, f)=|S(\tau, f)|^{2}
$$

The selection of the window function affects the time and frequency resolutions of the signal decomposition. Small time resolution, or a narrow the time window function, leads to a wider frequency resolution in its spectrum. However, according to uncertainty principle (see Section 4.2.1), the time and frequency cannot be chosen arbitrary at the same time. The time and frequency resolutions of a window function are dependent on the parameter $\tau$. This means that when the window function is chosen, the time and frequency resolution over the entire time-frequency plane is permanent. Figure 4.1 shows the state where the area defined by $\Delta f \times \Delta \tau$ remains the same over the time -frequency domain. This characteristic will be further investigated in more detail in the wavelet transform discussed in the next section. 


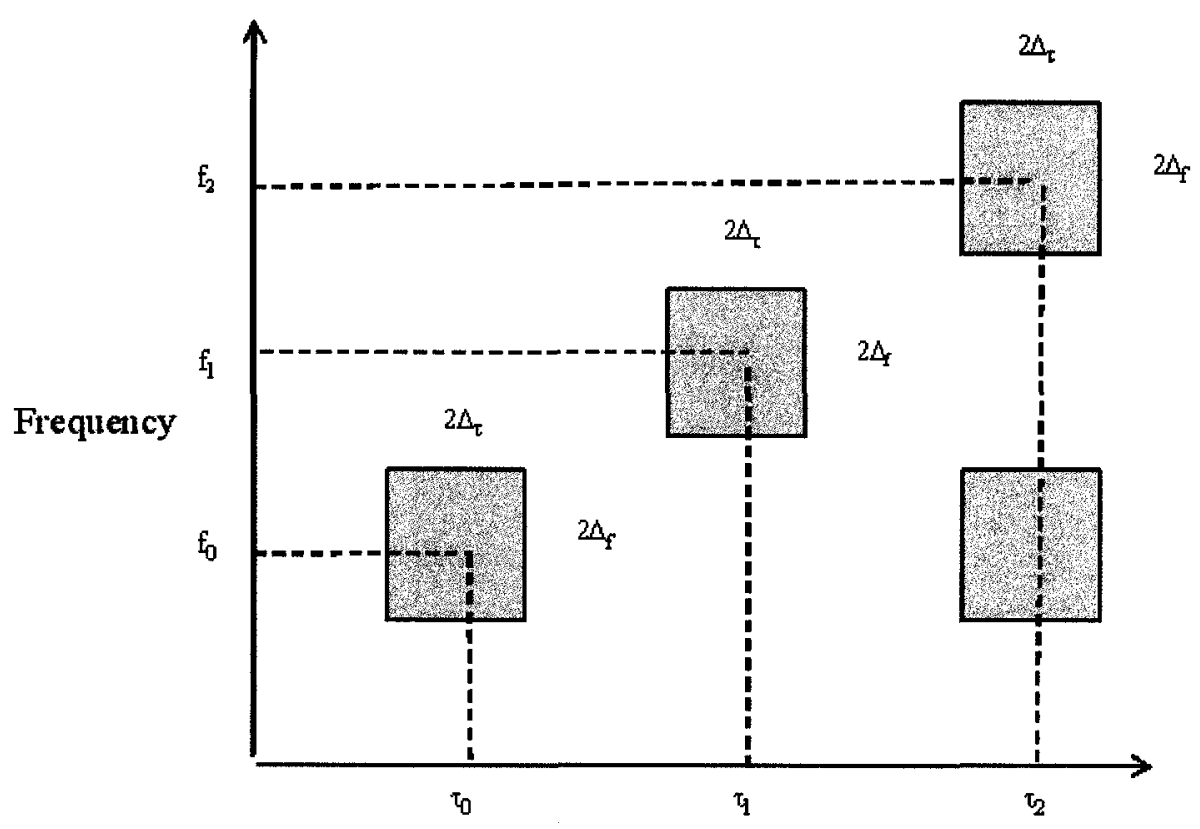

Time

Figure 4.1 Time and frequency resolutions of the STFT

\subsubsection{Wavelet transform}

The concept of wavelet in its present form was proposed by Jean Morlet (Hubbard, 1998). The method of wavelet analysis has been developed mainly by Y. Meyer (1993) and his colleagues, who have ensured the methods' dissemination. The main algorithm dates back to the work by Mallat (1998). Daubechies (1998) and other researches have recently been actively working in this field.

\subsubsection{Continuous Wavelet Transform}

In contract to STFT where the window size is fixed, the wavelet transform enables variable window sizes in analysing different frequency components within a signal; small window for high frequencies and large windows for low 
frequencies. The result is a constant relative-bandwidth frequency analysis, which is well suited for capturing transient features in time-varying signal. Unlike the FFT, which expresses a signal as the sum of a series of single frequency sine and cosine functions, the wavelet transform decomposes the signal with respect to a chosen continuous function, $\psi(t)$, called "mother wavelet". It transforms a continuous signal, $f(t)$ in to a function $W_{c}(s, \tau)$ of two real continuous variables, scale, $s$ and translation, $\tau$ called wavelet coefficients:

$$
W_{c}(s, \tau)=\int_{-\infty}^{\infty} f(t) \psi_{s, \tau}(t) d t
$$

where $f(t)$ belongs to the space of square integrable functions. In other words, it has finite energy condition, i.e.;

$$
\int|f(t)|^{2} d t \leq \infty
$$

$\psi_{s, \tau}(t)$ Corresponds to versions of wavelet function that has been shifted by the translation $\tau$, and dilated by the scale parameter $\mathrm{s}$, as given by:

$$
\psi_{s, \tau}(t)=\frac{1}{\sqrt{s}} \psi\left(\frac{t-\tau}{s}\right)
$$


Note that $\psi(t)$ must be oscillatory and have zero average, i.e.,

$$
\int_{-\infty}^{\infty} \psi(t) d t=0
$$

The parameter $s$ determines the time and frequency resolutions of the scaled mother wavelet, $\psi\left(\frac{t-\tau}{s}\right)$.

The time and frequency resolutions are directly and inversely proportional to the scaling parameter $s$. In Figure 4.2, variation of the time and frequency resolution at three locations has been illustrated. Changing the scale from $s_{0}$ to $s$ decreases the time resolution by half while doubling the frequency.

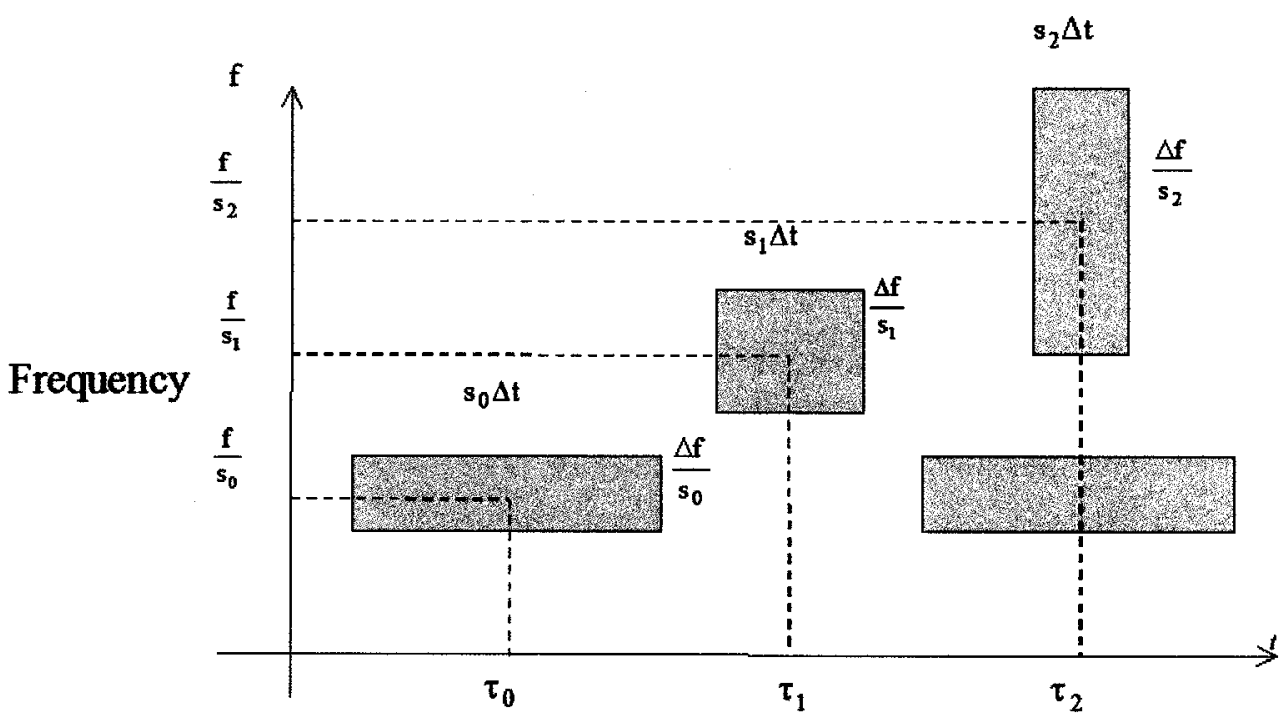

Time

Figure 4.2 Time and frequency resolutions of the wavelet transform

Some examples of mother wavelets are shown in Figure 4.3 for the so-called 
"Mexican hat" (Mex.hat) mother wavelet. It can be seen that as $s$ increases, the oscillations of the wavelet function "spread out" and the frequency of it in this local window decreases.

The wavelet transforms searches for similarity between the analyzed signal and the mother wavelet through the variation of the scales and time shifts of the mother wavelet function. For example, if the signal has a localized feature that matches closely with a particular version of the mother wavelet, $\psi_{s o, z o}(t)$, it accepts the value of the corresponding coefficients, $W_{c}\left(s_{0}, \tau_{0}\right)$ to be large. By applying the wavelet transform, extracting signal features is possible even when the signal does not have a dominant frequency, as required in the FFT case. However, since both $s$ and $\tau$ are continuous, the CWT can be a highly redundant transform.

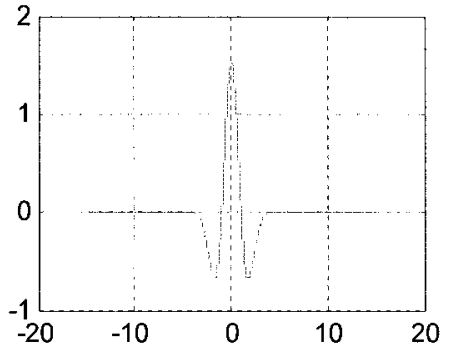

(a)

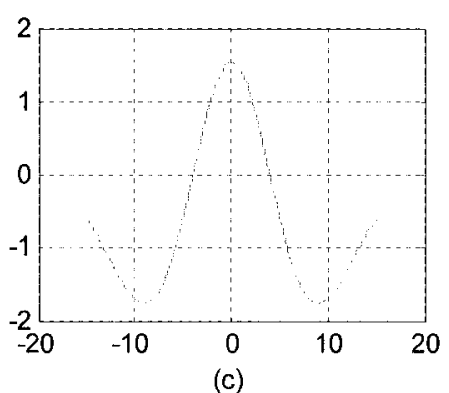

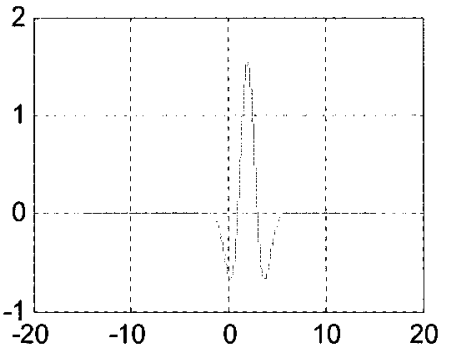

(b)

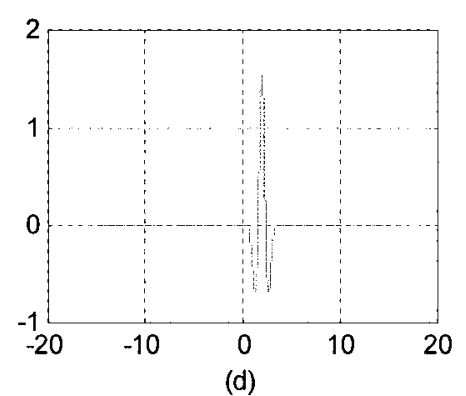

Figure 4.3(a)Original Mex.hat, (b)Translated Mex.hat, $(\tau=2)$,(c) dilated Mex.hat ( $\mathrm{s}=2)$, (d) Translated and scaled Mex.hat $(\tau=2, \mathrm{~s}=0.2)$ 


\subsubsection{Envelope Spectra of Wavelet Transform}

In the envelope analysis of a faulty bearing signal the resonant frequency band in which the impulsive components excited by defects are dominant has to be selected. However, as Dadouche et al (2007) reported, it is difficult to predict which resonance modes of the adjacent structures will be excited by the defects. The wavelet transform can be used as a band-pass filter to extract specific information from the vibration signal. Therefore, the wavelet transform can be applied on a signal as a series of filters to obtain resonant responses that contain the components of sought characteristic frequencies. Shi et al (2004) proposed a new approach based on the fusion of the wavelet transform, envelope spectrum and Hilbert transform ${ }^{1}$ to detect and localize defects in the rolling element bearing. In addition, Yan et al (2005) presented a demodulation technique for defect detection on the bearings called Multi-Enveloping Spectrogram. They applied the Fourier transform on the modulus of the wavelet transform rather than Hilbert transform of the signal. By choosing a complex mother wavelet, the wavelet transform coefficients are also complex and naturally analytic ${ }^{2}$. Then, the envelope waveform can be generated calculating the modulus of $W_{c}(s, \tau)$. Given $\left.W_{c r}(s, \tau)\right)$ and $W_{c_{i}}(s, \tau)$ as the real and imaginary part of the $W_{c}(s, \tau)$, respectively:

$$
M W_{c}(s, \tau)=\sqrt{W_{c r}(s, \tau)+W_{c i}(s, \tau)}
$$

Therefore, the wavelet-based envelope spectrum can be acquired by calculating the Fourier Transform of the envelope waveform of $M W_{c}(s, \tau)$ :

\footnotetext{
${ }^{1}$ Applying Hilbert transform on a signal generates the corresponding analytic signal.

${ }^{2}$ The real and imaginary parts are the original and the Hilbert transform of it, respectively.
} 


$$
A_{c}(s, \omega)=\int_{-\infty}^{+\infty} M W_{c}(s, \tau) e^{-\mathrm{j} \omega \tau} d \tau
$$

\subsection{Summary}

Time -domain analysis, overall vibration level and waveform metrics such as crest factor and kurtosis can provide basic diagnosis facts on rolling element bearing in simple machineries. However, time domain metrics fail to diagnose the small size defects because of background noise in the signal. Among the frequency- domain techniques, envelope analysis provides satisfactory results to localize the defect on the bearings for few operating conditions in machines. Time-frequency domain techniques, especially with the combined the envelope analysis, offer the opportunities to identify the health condition of bearings and simplifies the diagnosis of defects on the bearings.

Based on the above, this thesis will concentrate on analyzing the defective component of bearings by the time-domain, frequency-domain and envelope spectra using wavelet transform analysis. 


\section{Chapter 5}

\section{Signal Analysis and Data Comparison}

This Chapter presents a comparative study of four distinct signal processing techniques commonly employed for non-stationary signal processing. The analytical framework of the time domain, frequency domain, and wavelet transforms were presented in details in Chapter 4.

The effectiveness of each technique in detecting transient features from a timevarying signal with different operational conditions of speed and load will be examined. Subsequently, the performance of each technique is evaluated, using realistic vibration and acoustic signals measured from a bearing test rig described in Chapter 3. However, defects on the outer race were easily detectable than the ones on the inner race and rollers. This was mainly due to the fact that the outer race is stationary and the sensors were easily mounted close to the defect location. One the other hand, the motion of the rollers and inner race affect signal strength since defects on those components pass through the bearing loaded zone only every single cycle of the shaft. Therefore, this chapter discusses the impact of load and speed on the fault detectability for defects located on the outer race and analyses the defects on the inner race and roller for one specific operating condition.

The test-rig was operated at three different rotational speeds; 900, 1800 and $2100 \mathrm{rpm}$. For each rotational speed three load cases were considered: 100, 250, and 500lbf. For every test condition a total of 3 data files were recorded to ensure the repeatability of the tests. 
The following format was employed for labeling all the data presented next.

L1; L2; L3; L =load; $1=$ load value of $100 \mathrm{lbf} ; 2$ =Load value of 250lbf;

$3=$ Load value of $5001 \mathrm{bf}$.

$\mathbf{9}=900 \mathrm{rpm} ; \mathbf{1 8}=1800 \mathrm{rpm} ; \mathbf{2 1}=2100 \mathrm{rpm}$

$\mathbf{B}=$ Healthy Bearing; $\mathbf{O}=$ Defective Outer race; $\mathbf{I}=$ Defective inner race;

$\mathbf{R}=$ Defective Roller

as some examples:

'B9L1'= Healthy bearing at 900rpm and 100lbf.

'O18L2'= Outer race defect at 1800rpm and 250lbf.

'I21L3'=Inner race defect at 2100rpm and 500lbf

'R18L1'= Rolling defect at 1800rpm and 100lbf.

\subsection{Defective Outer Race Analysis}

As mentioned earlier, an accelerometer was used in this study as a reference sensor for comparison purposes with the ACUT, Eddy current probe, and PUT.

A collection of accelerometer (radial), ACUT, Eddy current and PUT time signatures with a noise background of undamaged and damaged outer race is shown in Figures 5.1 to 5.4. The signals show little information on the effect of the damage size. For example, as the simulated damage grows, there is an increase in overall signal amplitude and noise floor. The time history of axial accelerometer is very similar to the one of the radial accelerometer (Appendix A). 

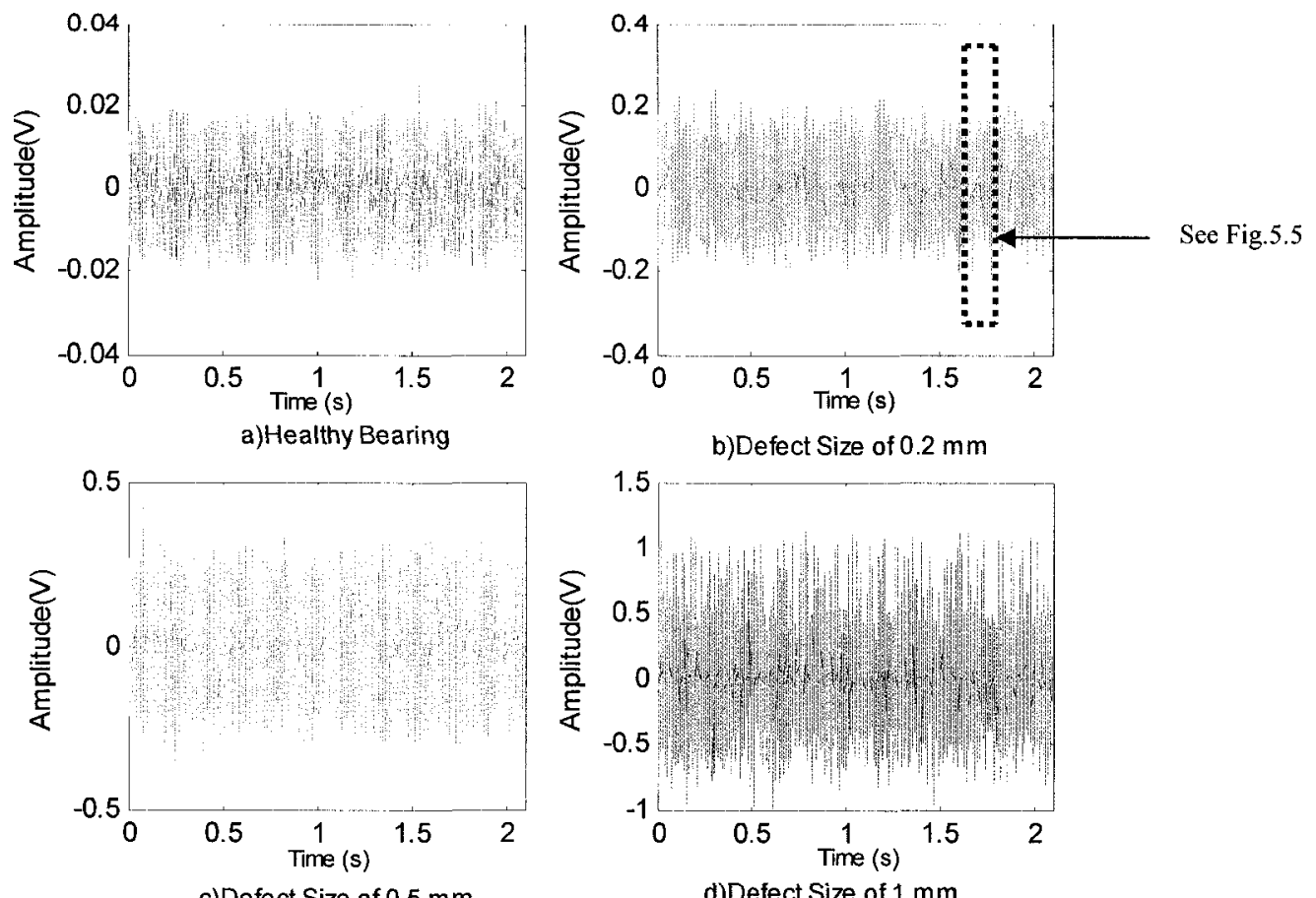

Figure 5.1 Typical radial accelerometer signal for outer race, 1800rpm, 500lbf
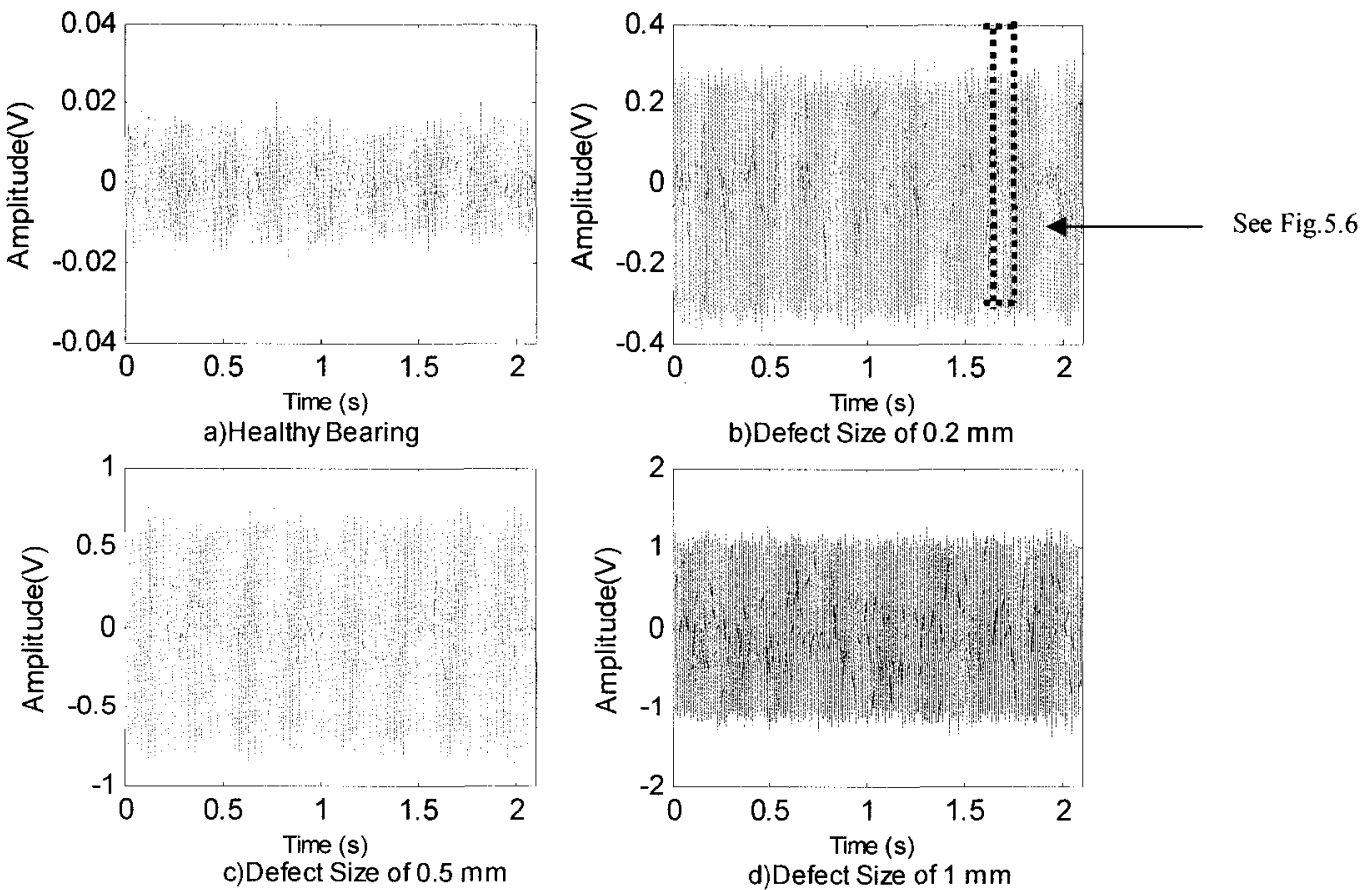

Figure 5.2 Typical ACUT signal for outer race, 1800rpm, 500lbf 

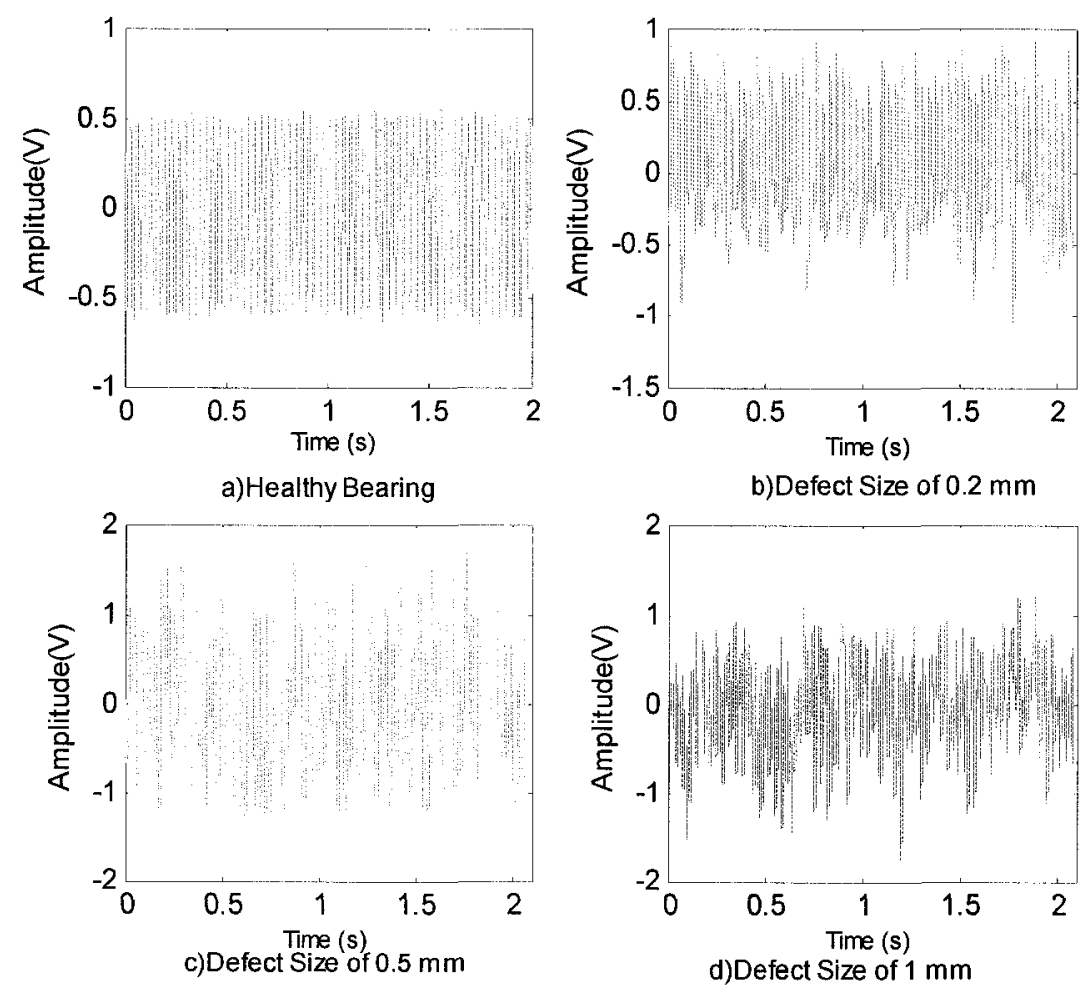

Figure 5.3 Typical Eddy current signal for outer race, 1800rpm, 500lbf
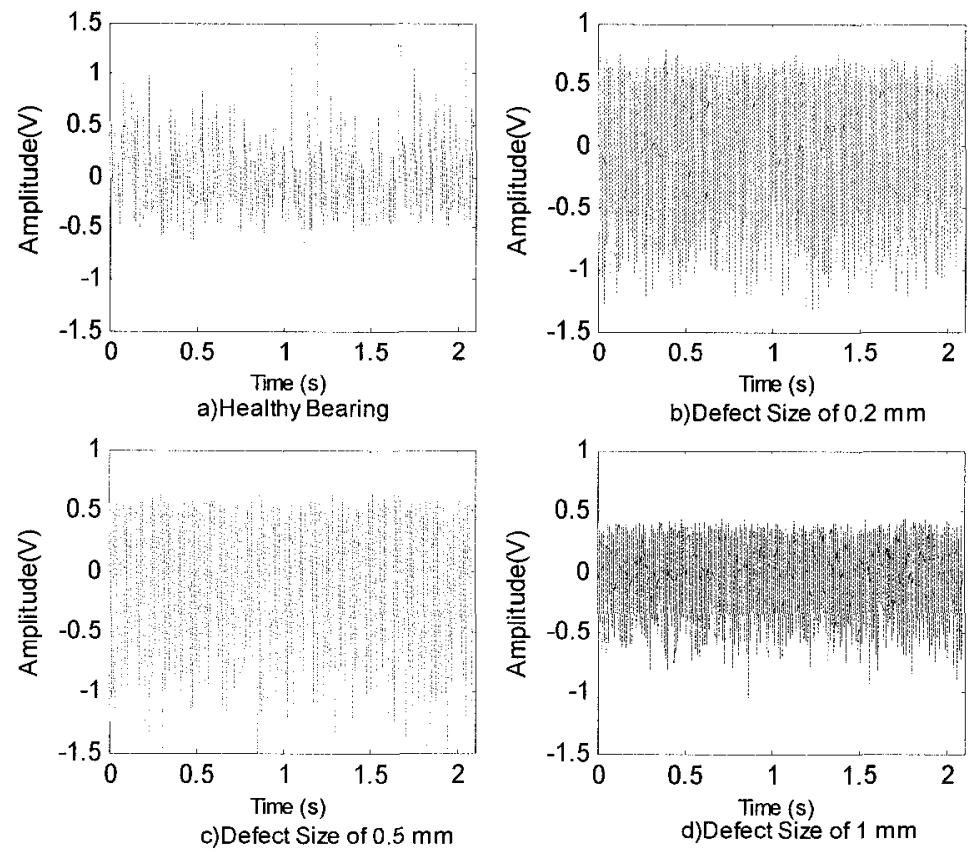

Figure 5.4 Typical PUT signal for outer race, 1800rpm, 500lbf 
Figures 5.5 and 5.6 represent close-up signatures of Figures 5.1 and 5.2 respectively, primarily to provide evidence that the spiky part of the signatures is not attributed to spurious electronic noise and to demonstrate that the impulses are due to the contact of the defect to the adjacent surfaces. The close-up of other time histories shows the same trend and are not shown here.

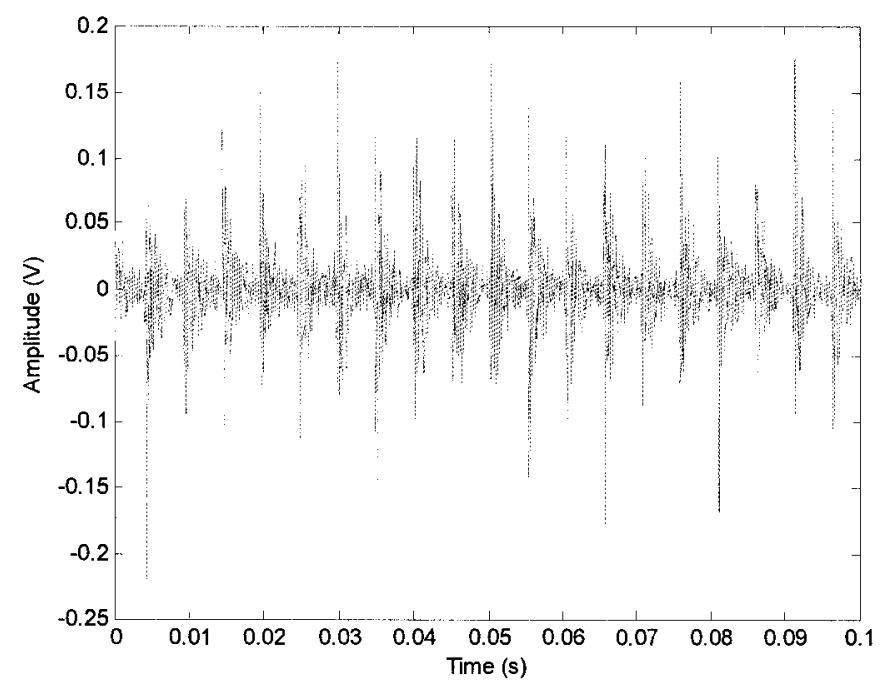

Figure 5.5 Typical accelerometer signal for defective outer race, 1800rpm, 500lbf (zoom of Figure 5.1(b))

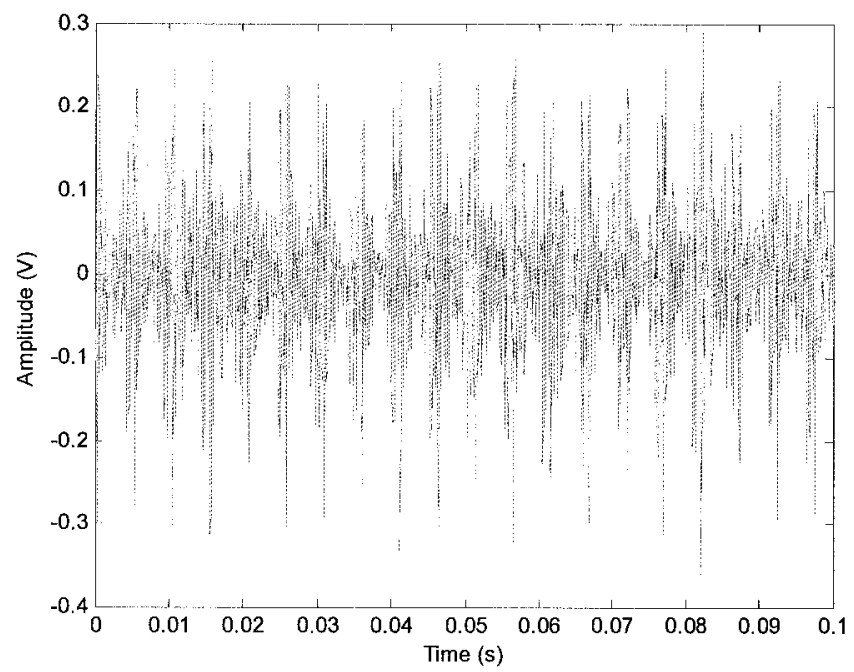

Figure 5.6 Typical ACUT signal for defective outer race, 1800rpm, 5001bf (zoom of Figure 5.2(b)) 


\subsubsection{Time-domain Analysis}

Because of the large number of records that took place during the tests (stored at the regular interval of 5 minutes at the approximately 120 hours), the trend of the time is possible.

\subsubsection{Accelerometer}

The 'time domain' signal metrics of peak and RMS values for the accelerometer at the three different speeds (900rpm, 1800rpm, 2100rpm) and loads (100lbf, 250lbf, 500lbf) with a growing defect on outer race are shown in Figure 5.7 and 5.8. Typically, the peak value and RMS increase with increasing load for bigger defect sizes. However, speed and defect size significantly affect those time metric parameters. The value of crest factor at speeds of 900, 1800, and $2100 \mathrm{rpm}$ and a constant load of $100 \mathrm{lb}$ is plotted in Figure 5.9. The Figure indicates that this factor is very poor in tracking the defect size. Tandon (1994) has also reported that the crest factor is not a good indicator of failure in rolling element bearings.

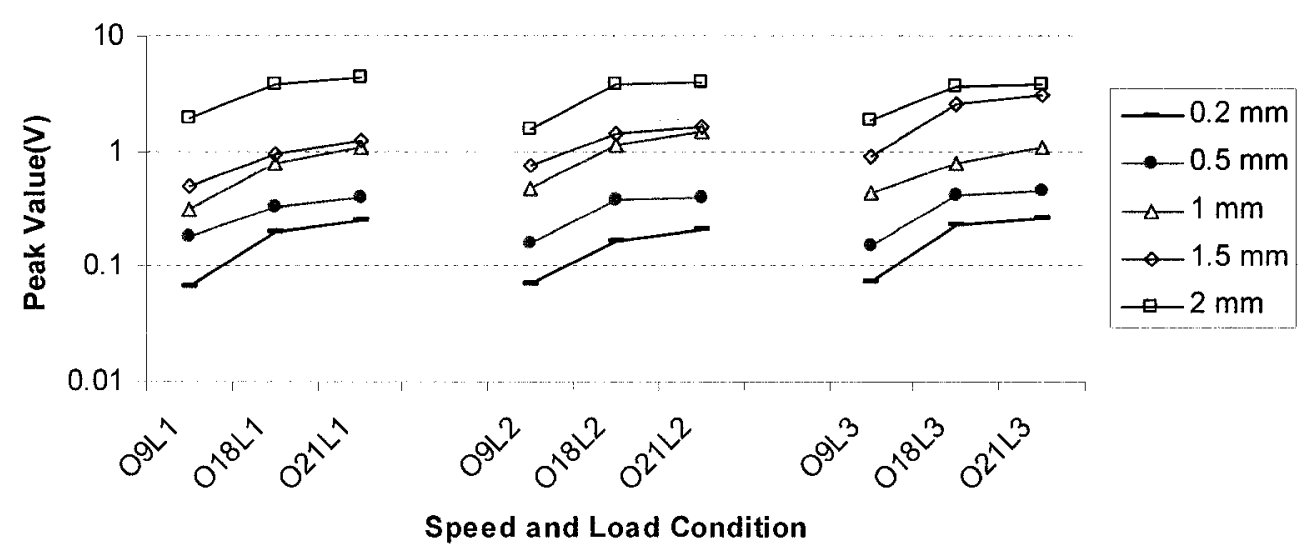

Figure 5.7 Accelerometer peak values for outer race defects 


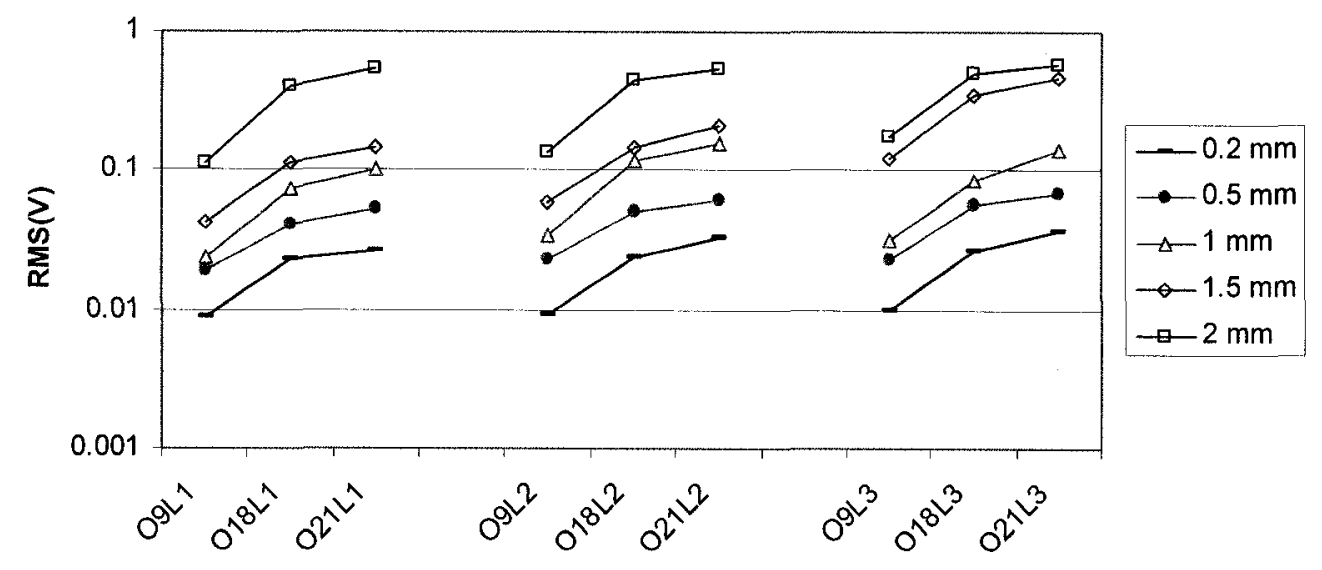

Figure 5.8 Accelerometer RMS values for outer race defects

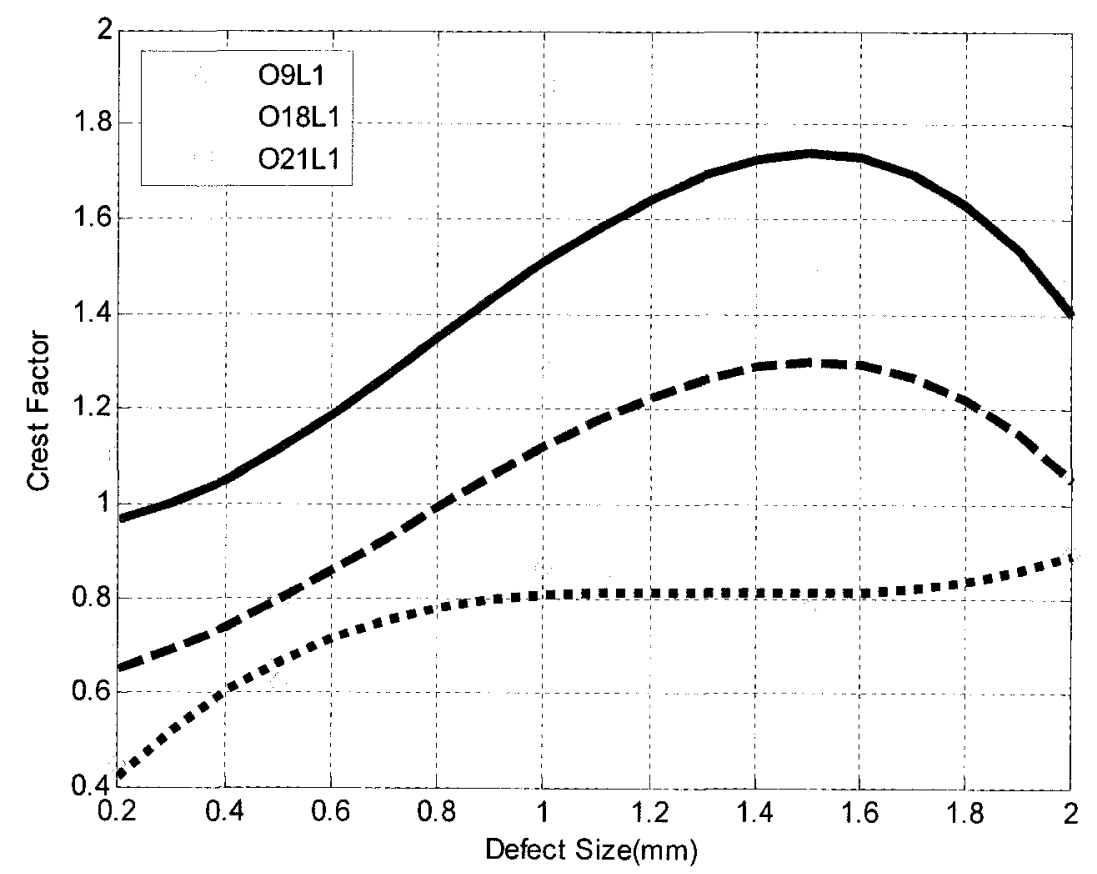

Figure 5.9 Accelerometer crest factor for outer race defects as a function of defect size 


\subsubsection{Air Coupled Ultrasonic Transducer}

Figure 5.10 shows the RMS values obtained from the ACUT raw signal. For smaller defect sizes of 0.2 and $0.5 \mathrm{~mm}$ and especially at low speed and load of respectively $900 \mathrm{rpm}$ and $100 \mathrm{lbf}$, the RMS value could not track the defect growth. By increasing the defect size to $1 \mathrm{~mm}$, the defect is traceable for all remaining conditions. Figure 5.11 indicates the relationship between the defect size and ACUT RMS values. Surprisingly, as the defect progresses, the RMS value of ACUT decreases considerably for most of the measurements. This reduction is probably due to the increase of background noise. However, the ACUT RMS sensitivity to speed is significant, as shown on the plot.

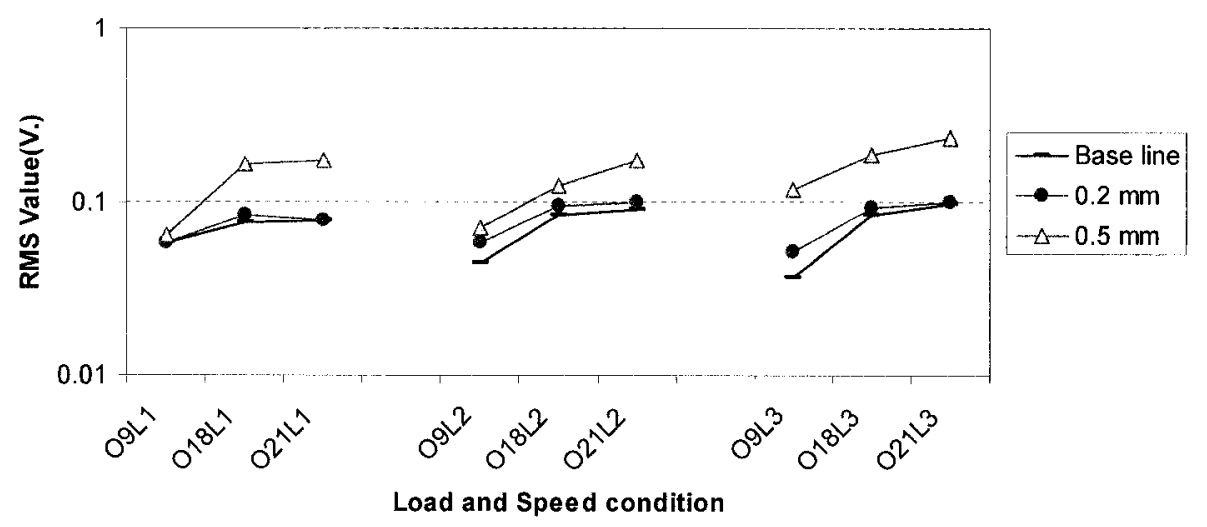

Figure 5.10 ACUT RMS Value for outer race defects 


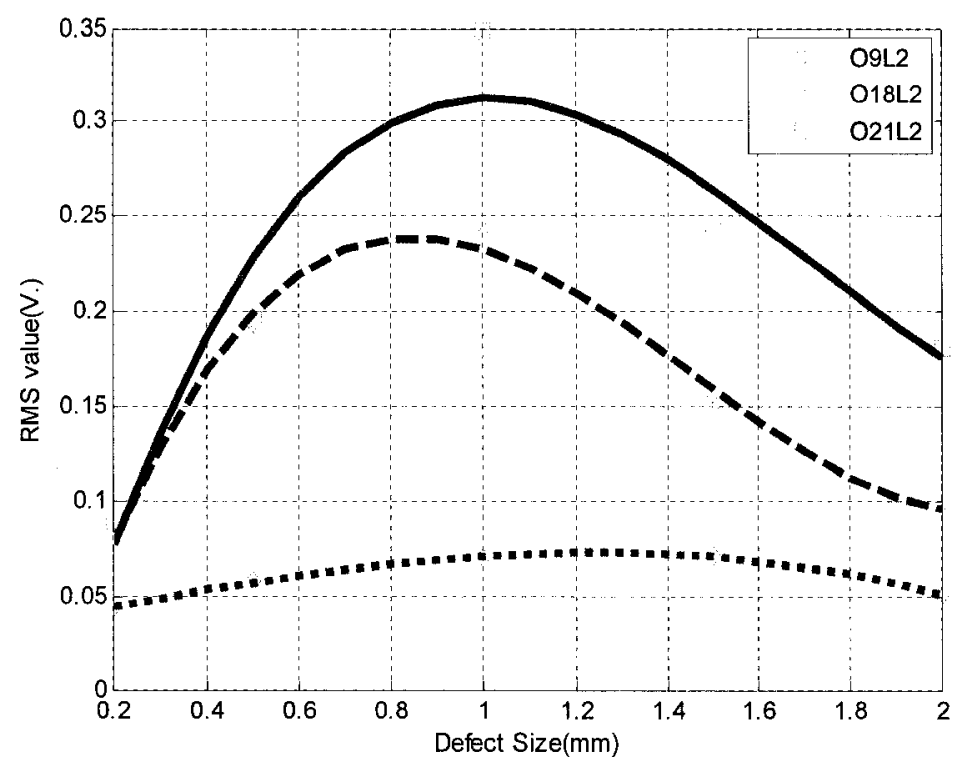

Figure 5.11 ACUT RMS values for outer race defects as a function of defect size

\subsubsection{Piezo Ultrasonic Transducer}

The trend of the peak value of the PUT with speed, load and defect size localized on the outer race is shown in Figure 5.12. As the Figure shows, and for most cases, the peak value decreases when speed increases. The peak value is more sensitive to defects larger than $0.5 \mathrm{~mm}$ than to smaller ones. In addition, increasing the bearing load does not affect much the peak value. Figure 5.13 illustrates the relationship between PUT RMS value and speed for distinct defect sizes on the outer race. Similarly to the peak value, for a fixed load, increasing the rotational speed results in a decrease of the RMS value. 


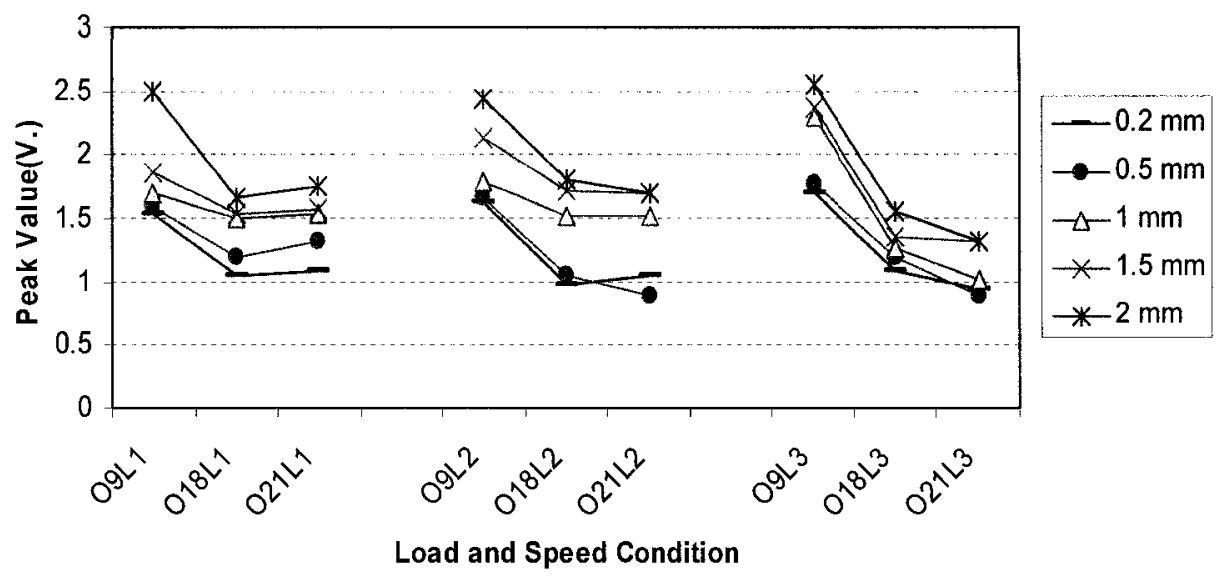

Figure 5.12 PUT Peak value for outer race defects

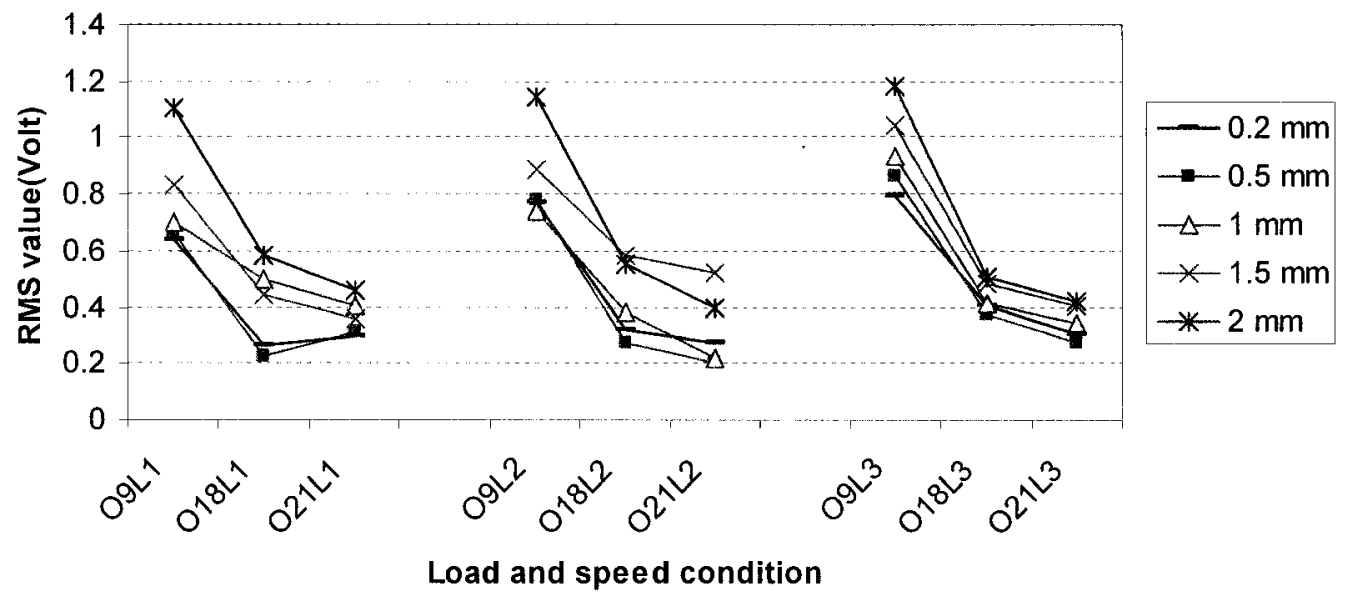

Figure 5.13 PUT RMS value for outer race defects 


\subsubsection{Frequency- Domain}

\subsubsection{Accelerometer}

Figure 5.14 shows the corresponding spectra for the raw time signal of radial vibrations (accelerometer) presented in Figure 5.1 for healthy and faulty bearings at $1800 \mathrm{rpm}$ and 500lbf up to the Nyquist frequency. Contrary to the time signal, the spectra give a little more information on the effect of the damage size. The peaks on the spectra represent the resonance frequencies of the system where the bearing is installed. The natural frequencies that are excited by the defect's impact are located between 1300 and $3500 \mathrm{~Hz}$. The amplitude ratio at the resonance frequency between faulty and healthy bearings is very significant. Besides, as the defect grows the amplitude of the dominant resonance frequency increases. The dominant resonance frequencies of axial vibrations are similar to those of the radial vibrations (see Appendix A).
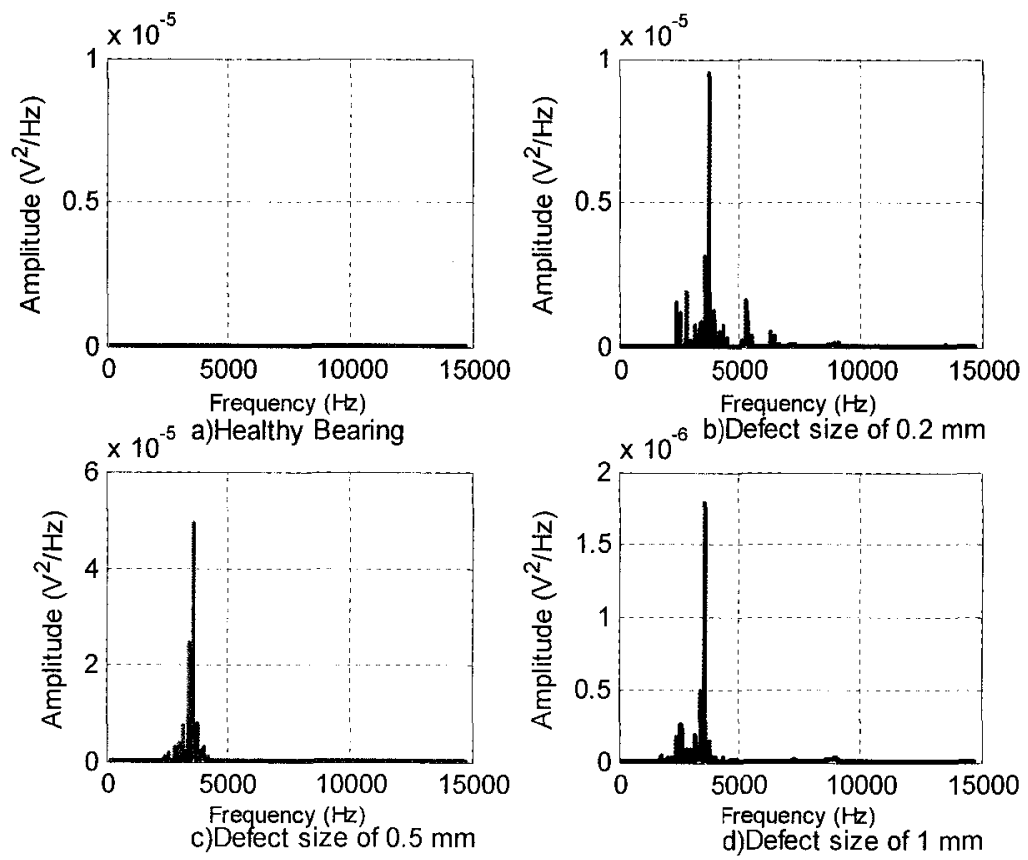

Figure 5.14 Accelerometer spectra of outer race, 1800rpm, 500lbf

As shown in Table 4.2, the bearing outer race fault related frequency, $f_{b p o r}$ at 
$1800 \mathrm{rpm}$ was $202.6 \mathrm{~Hz}$. Figure 5.15 shows the low-frequency range (up to $500 \mathrm{~Hz}$ ) of Figure 5.14. The spectra contain a variety of spectral components and harmonics of shaft rotation, which makes difficult the prediction of the bearing condition using vibration signature. The vibration spectrum does not necessarily reveal any information about the condition of the bearing.
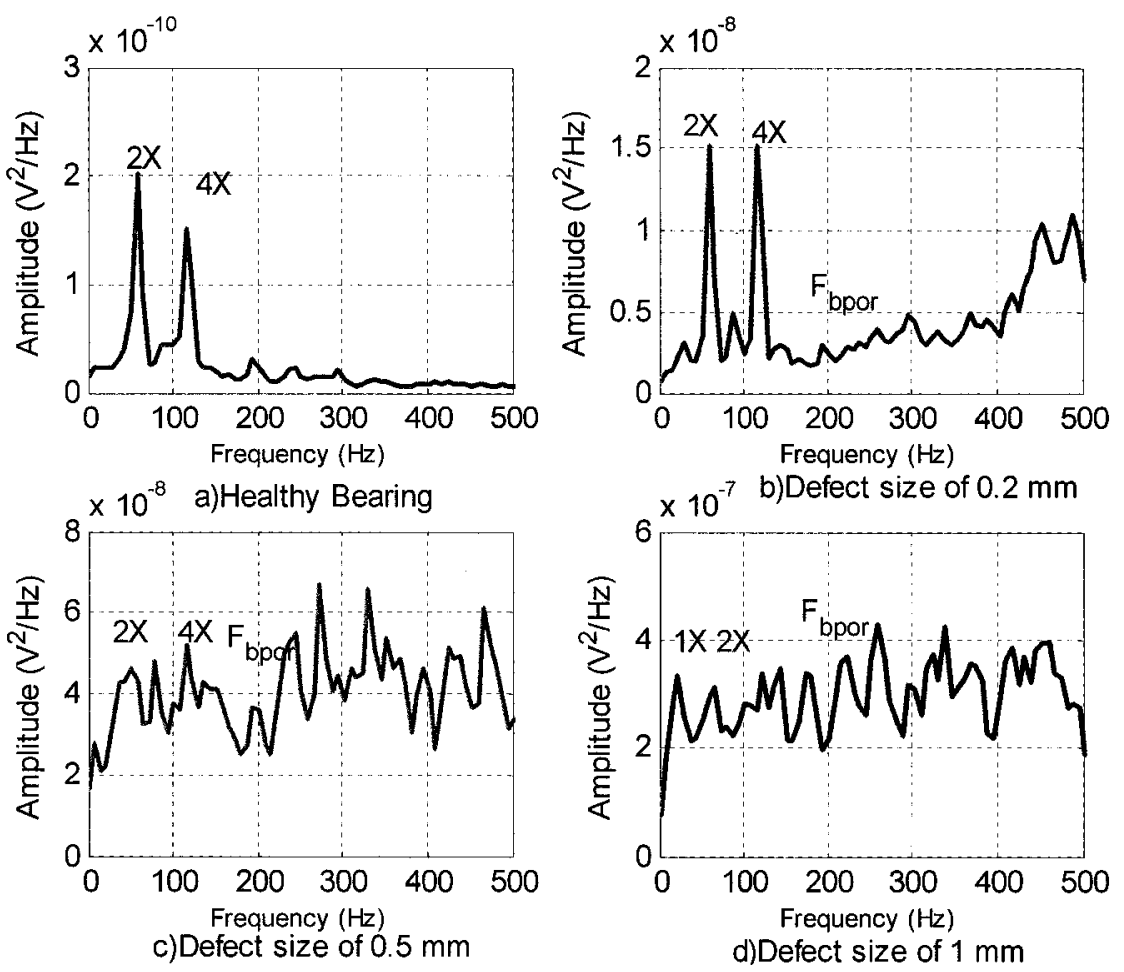

Figure 5.15 Acceleration spectra of outer race, 1800rpm, 500lbf, low- frequency range

Figure 5.16 compares the PSD at the bearing defect frequency of the baseline and faulty bearing in terms of defect size, shaft speed, and load. First, it can be seen that the PSD of the signal at the associated $f_{b p o r}$ increases with defect size and speed.

The spectral differences of normal and faulty bearing (defect size of $0.2 \mathrm{~mm}$ ) for all shaft speeds are most distinctive. For instance, at shaft speed of $900 \mathrm{rpm}$ and a load of $100 \mathrm{lbf}$, the vibration amplitudes of $f_{\text {bpor }}$ are $6.36 \mathrm{E}-12$ (healthy) and 
3.85E-10 (0.2 mm defective bearing), respectively. This represents a significant increase of 60 folds.

When the load was increased at a constant shaft frequency, both the normal and faulty, $f_{b p o r}$ spectral amplitudes differences were barely distinguishable. Hence, the influences of load on bearing fault PSD are generally, restricted by the vibration signatures.

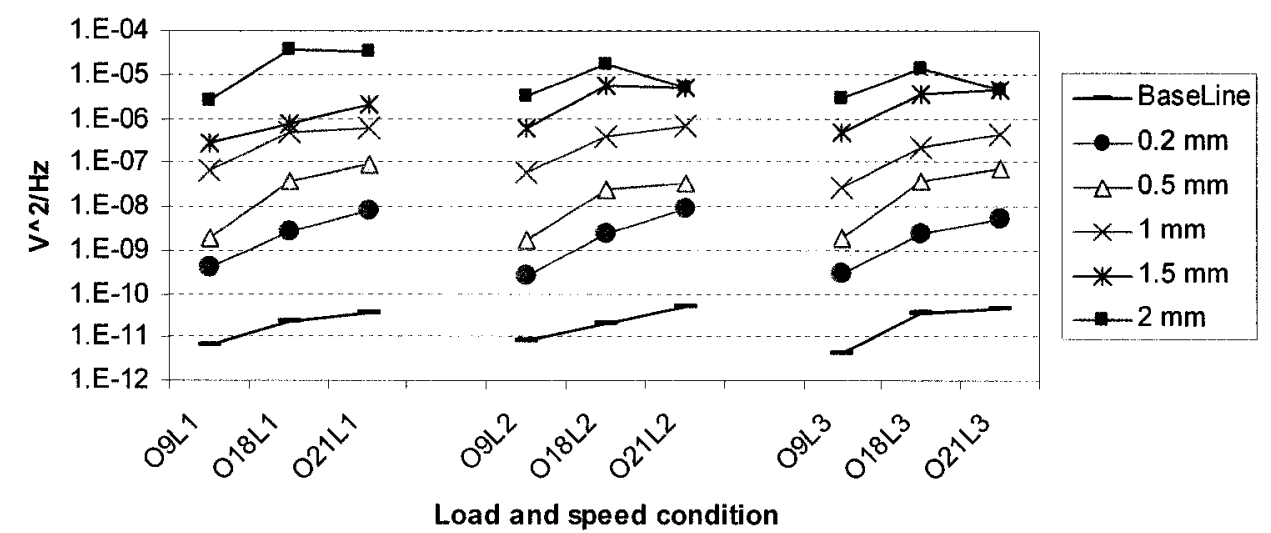

Figure 5.16 Effect of load and speed on radial vibration amplitude

\subsubsection{ACUT}

Figure 5.17 shows the spectra of the ACUT time history presented in Figure 5.2. As the accelerometer vibration spectra; the dominant frequencies are the resonance frequencies of the system. All the signals acquired with the ACUT for various operational conditions and several defect sizes have the same characteristics of high spikes at low and medium frequencies and relatively no peaks in the high frequency range. Furthermore, the carrier frequency was always found to be located between $1.9-3 \mathrm{kHz}$. 

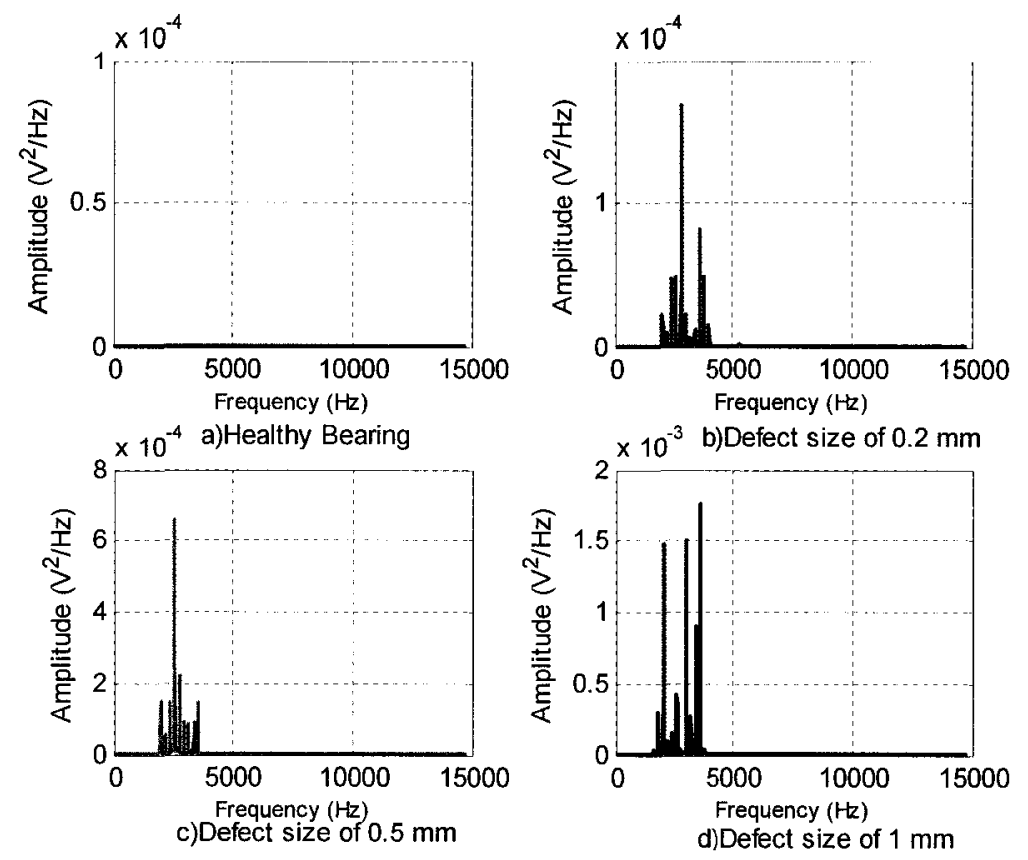

Figure 5.17 ACUT signal of outer race, 1800rpm, 500lbf

Figure 5.18 illustrates the low frequency range of Figure 4.17 (up to $500 \mathrm{~Hz}$ ). Acoustic spectra show very similar spectral shape to those of vibration signals in Figure 5.15 in terms of rotational harmonics and outer race fault. However, it is noted that the spectral amplitude of the baseline and faulty bearings is much higher than those of the vibration one. In addition, it is important to point out that acoustic signatures have much less noise in the low-frequency band, which represents an advantage for the ACUT. 

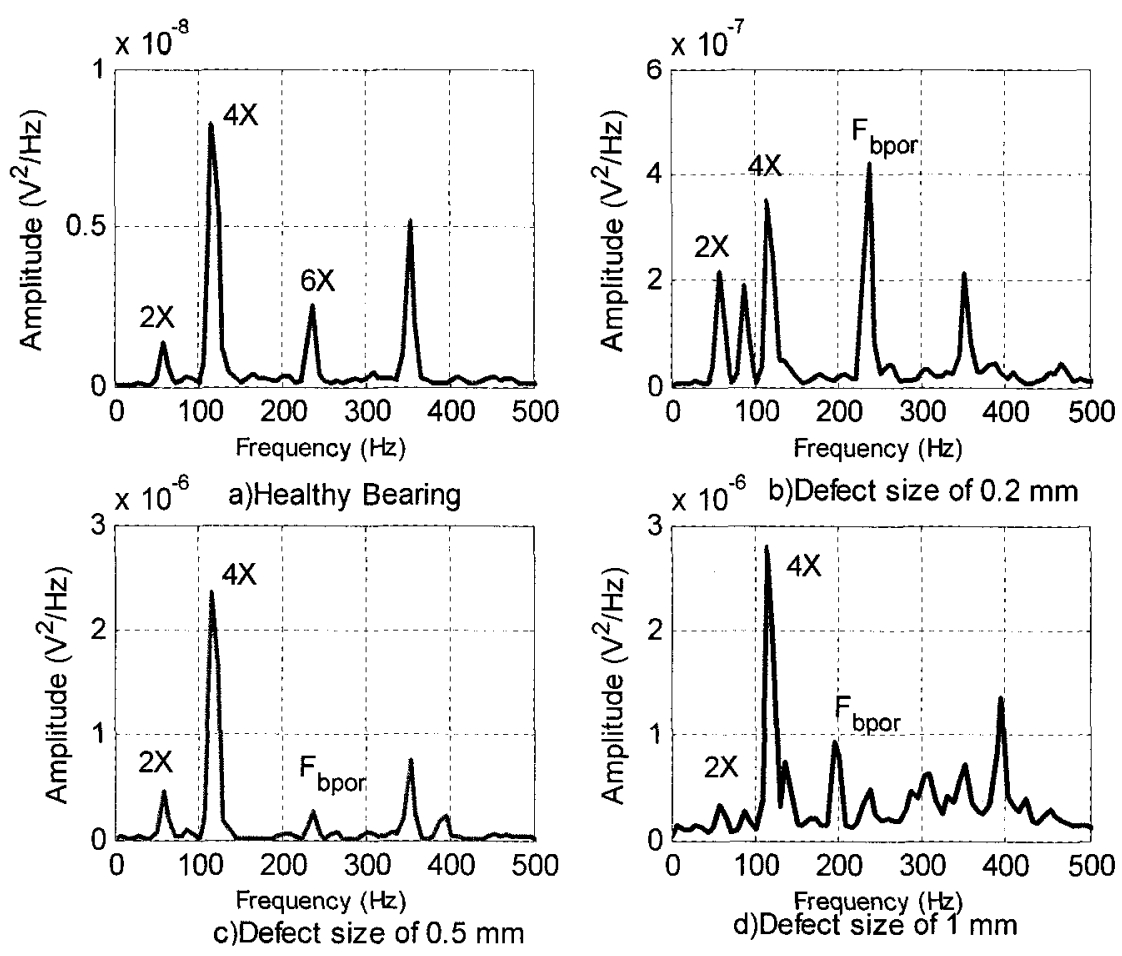

Figure 5.18 ACUT Spectra of outer race, 1800rpm, 500lbf, low frequency range

The effect of motor driving speed and load on the acoustic spectral amplitude at the specific defect frequency, $f_{b p o r}$ for healthy and defected bearings is illustrated in Figure 5.19. Similarly to the vibration signature spectra presented in Figure 5.16, the amplitudes increase with shaft speed. However, the amplitude differences between healthy and faulty bearings for a defect size of $0.2 \mathrm{~mm}$ are larger than those of vibration spectra. This represents another advantage to the ACUT and makes the acoustic analysis more efficient in detecting initial signs of cracks and defects on the outer race of rolling bearings.

On the other hand, the acoustic spectra is unable to differentiate between detect sizes of 0.2 and $0.5 \mathrm{~mm}$ and neither 1 nor $1.5 \mathrm{~mm}$. Therefore, vibration analysis seems to be more capable of identifying the defect size. 


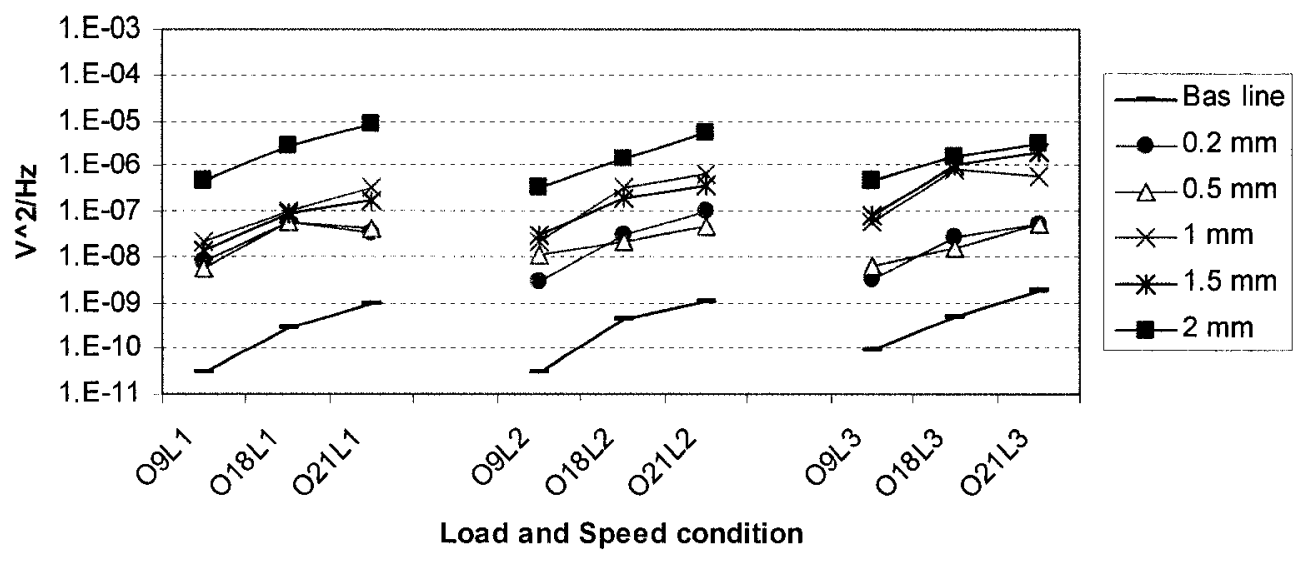

Figure 5.19 Effect of Load and speed on ACUT $f_{\text {bpor }}$ amplitude

\subsubsection{Eddy Current Probe}

Figure 5.20 shows the Eddy current probe PSD of healthy and faulty bearings at $1800 \mathrm{rpm}$ and 500lbf load. The Eddy current PSD also shows significant frequencies at the high and medium ranges of the spectra, which are signs that modes have been excited. The highest amplitude frequency for all cases with different defect sizes was around $1200 \mathrm{~Hz}$. The Eddy current PSD also shows significant frequencies at the high and medium ranges

Figure 5.21 illustrates the Eddy current spectra up to $500 \mathrm{~Hz}$. It is clear that the dominant frequencies were the motor driving frequency and its harmonics in the spectra. However, the Eddy current spectra will be later examined more using the HFRT technique. 

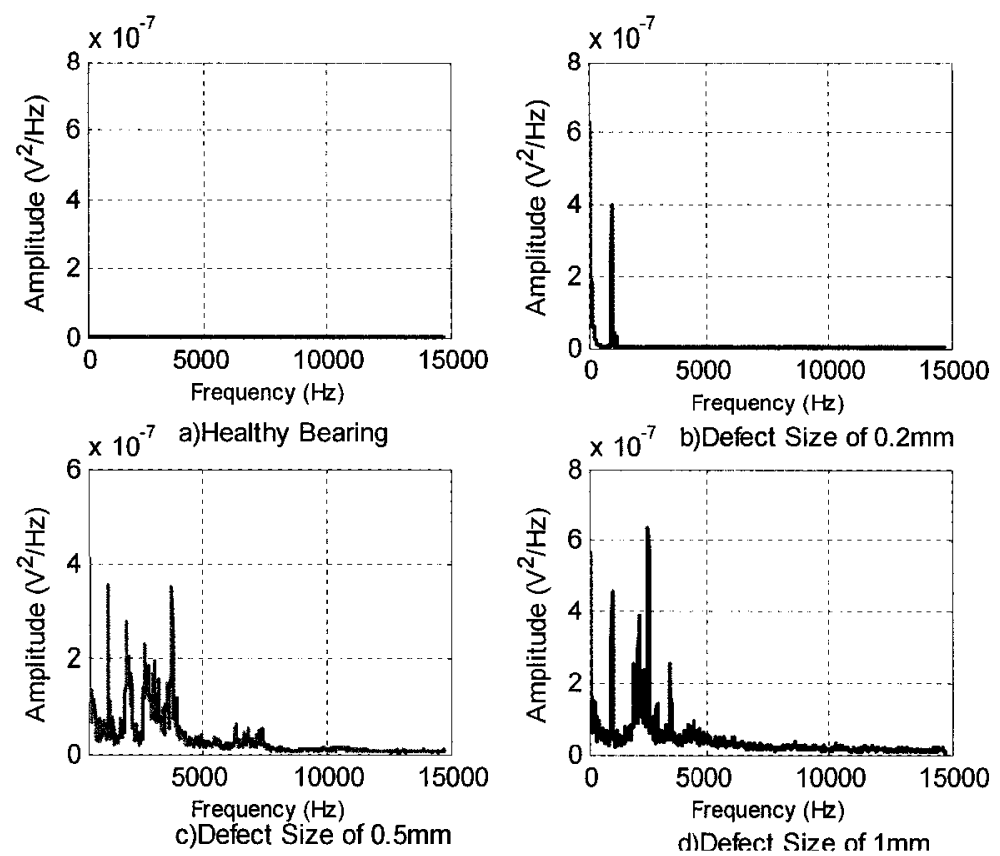

Figure 5.20 Eddy current spectra of outer race, $1800 \mathrm{rpm}, 500 \mathrm{lbf}$
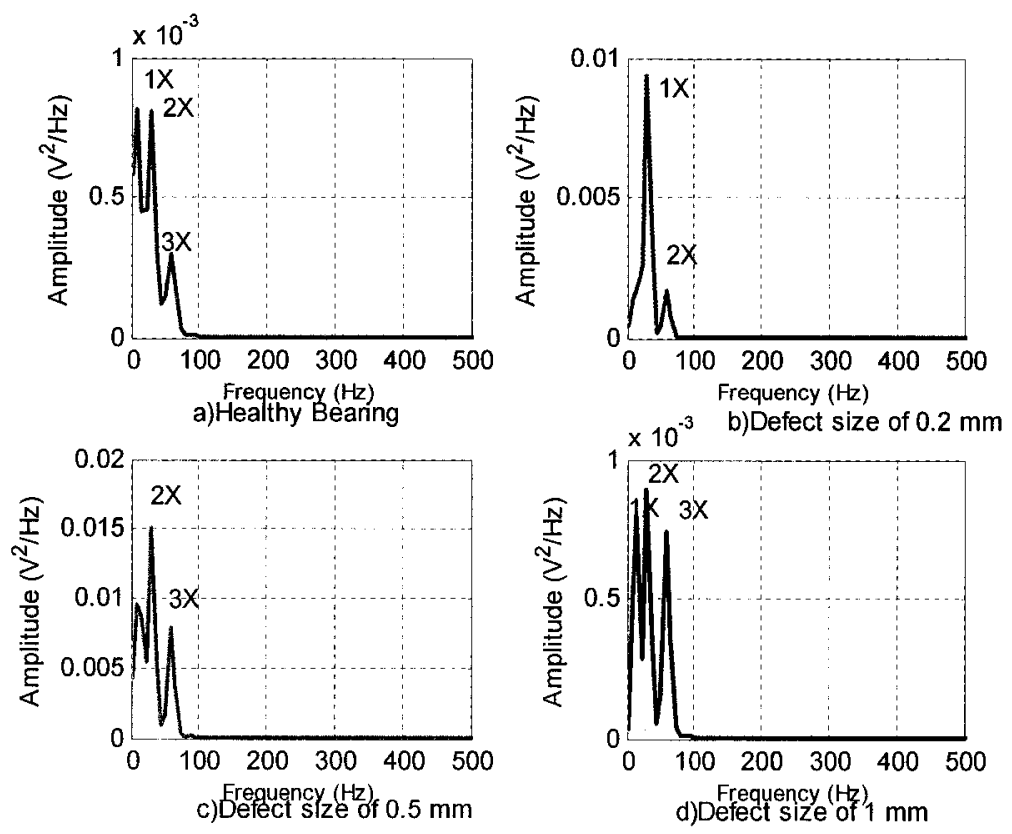

Figure 5.21 Eddy current spectra of outer race, 1800rpm, 500lbf 


\subsubsection{PUT}

Figure 5.22 illustrates the spectra of the corresponding PUT time signal of Figure 5.4. Despite the signal was sampled at higher rate $(30 \mathrm{kHz})$, the higher and medium range of frequencies does not show any sign of excitation or defect signature. However, the lower frequency range shows very meaningful information. First, in Figure 5.22(a), the Shaft frequency component is the highest in the healthy bearing spectrum and there is no sign of the defect frequency, $f_{b p o r}$. Secondly, the visible peaks at $f_{\text {bpor }}(202 \mathrm{~Hz})$ are present in the spectra of defective bearings with defect widths of $0.2,0.5$, and $1 \mathrm{~mm}$. These represent unique results. This sensor was the only one, among the studied sensors, capable to detect the defect on the bearing outer race in the frequency domain just by inspection of the PSD raw signal.
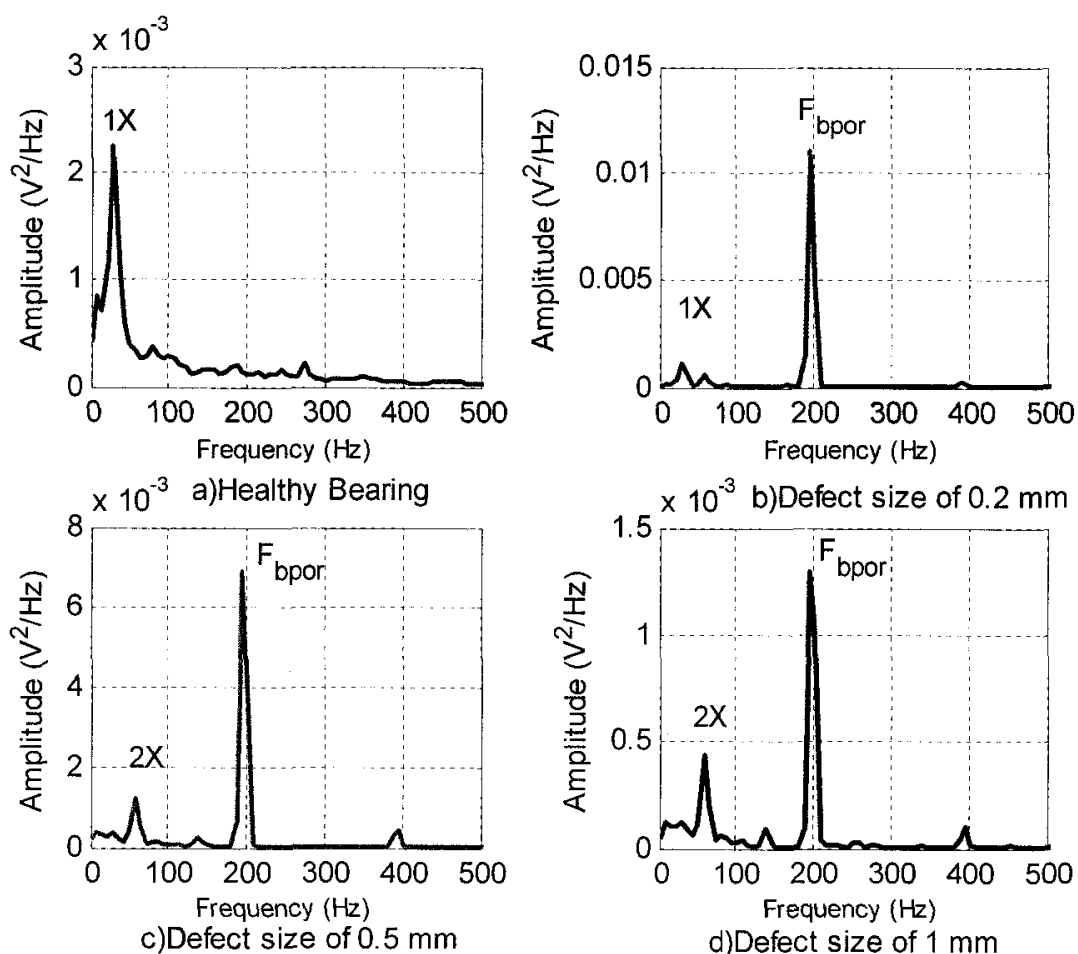

Figure 5.22 PUT signal spectra of outer race, 1800rpm, 500lbf, low- frequency range 
The spectral comparison between healthy and faulty bearings with different defect sizes is shown in Figure 5.23. Contrary to the accelerometer and ACUT, increasing the speed results in a decrease of the spectral amplitude. It is also important to notice that for most studied cases (defect width of $0.2 \mathrm{~mm}$ ) there was a significant change in power between healthy and defective bearings except for the case of a shaft speed of $2100 \mathrm{rpm}$ and a load of $250 \mathrm{lbf}$ where the difference is negligible.

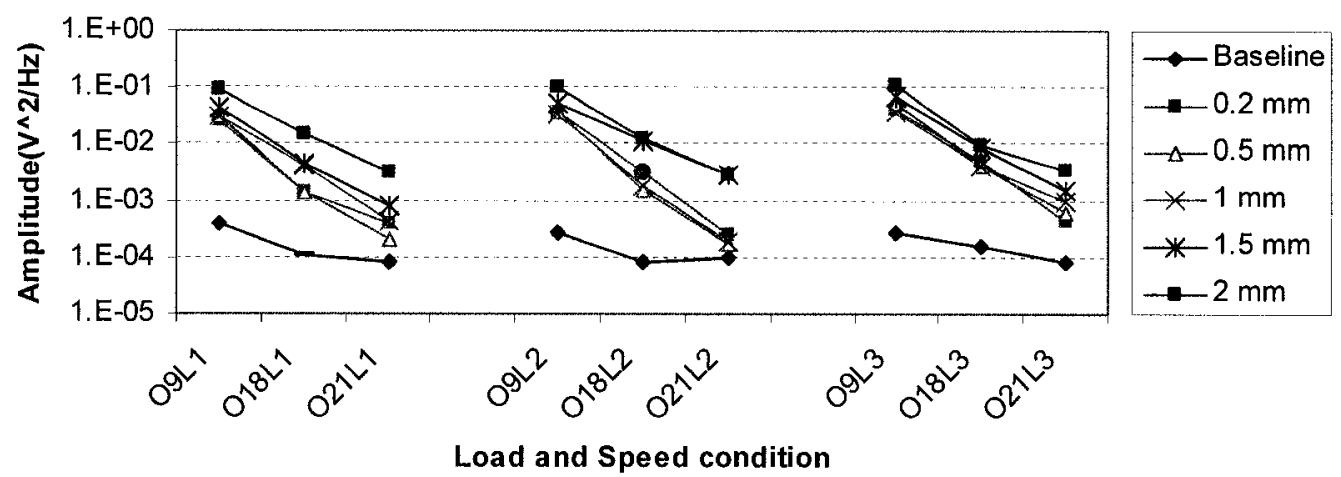

Figure 5.23 Effect of Load and speed on PUT, $f_{\text {bpor }}$ amplitude

\subsubsection{Envelope Analysis}

Figure 5.24 shows the PSD of the vibration demodulated signals previously presented in Figure 5.1 for a healthy bearing and defective ones (defects of 0.2 , 0.5 , and $1 \mathrm{~mm}$ wide located on the outer race). This demodulation procedure is based on the selection of the dominant frequency presented in the simple PSD of the raw signal (Figure 5.14), as a cut off frequency in the high-pass or band-pass filters. For instance, for a defect size of $0.2 \mathrm{~mm}$, a band-pass filter with a cut off frequency of $3 \mathrm{kHz}$ was used in the envelope detection procedure. After demodulating the filtered signal and estimating its PSD, the dominant frequency, as shown in Figure 5.24(b), is $194 \mathrm{~Hz}$, which is $8 \mathrm{~Hz}$ smaller than the defect frequency of $202 \mathrm{~Hz}$. Since the operational speed was always fluctuating 
$( \pm 20 \mathrm{rpm})$, this dominant frequency can be taken as the defect frequency, $f_{\text {bpor }}$. Defects with a width of 0.5 and $1 \mathrm{~mm}$ were also detectable applying the same algorithm. The amplitudes of $f_{\text {bpor }}$ are $1.58 \times 10^{-6}$ for defect size of $0.2 \mathrm{~mm}$, $2.19 \times 10^{-5}$ for defect size of $0.5 \mathrm{~mm}$, and $6.47 \times 10^{-5}$ for defect size of $1 \mathrm{~mm}$, respectively. Hence, the spectral amplitude of the outer race defect frequency increases when the defect size develops and grows.

Moreover, envelope detection was also performed using a band-pass filter centered at the frequency of $3 \mathrm{kHz}$ for all the operating conditions tested (different speeds and loads) and the results were quite similar to those obtained using the high-pass filter. It is important to mention that a $16^{\text {th }}$ order IIR Butterworth digital band-pass filter was selected for use with the HFRT algorithm.

The envelope procedure was also applied on the baseline signal (healthy bearing) and, as expected, there was no sign of the defect frequency on the spectrum as depicted in Figure 5.24(a).
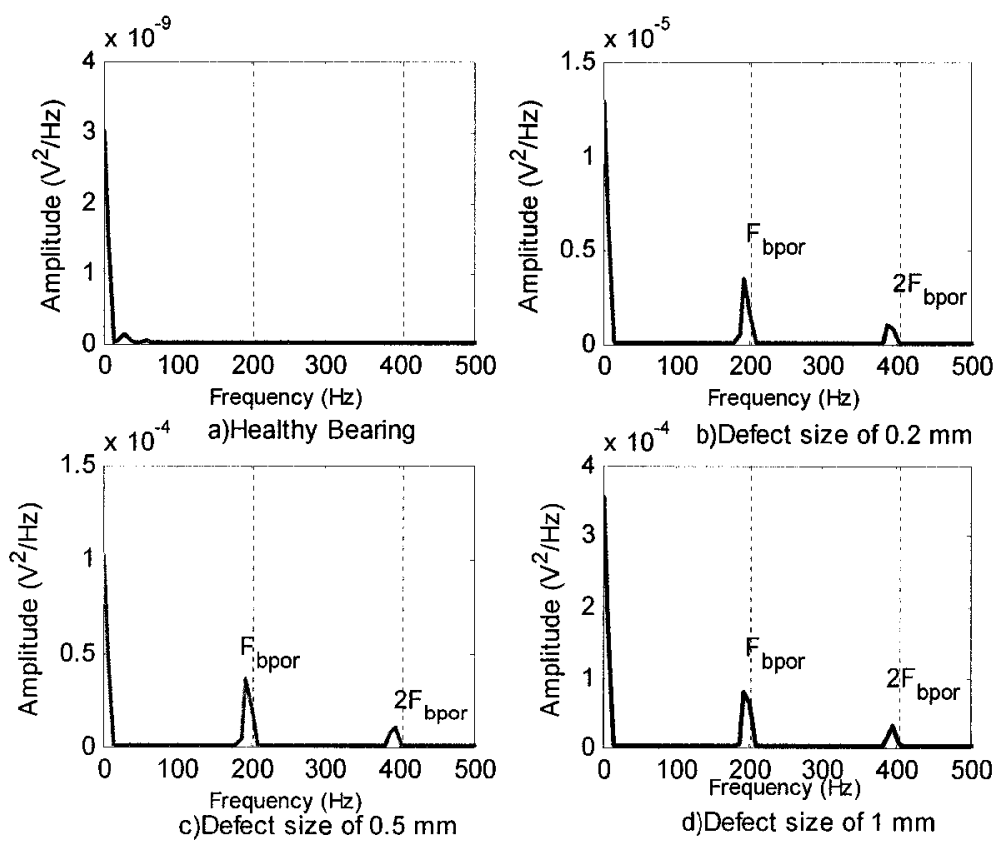

Figure 5.24 Accelerometer demodulated signal spectra of outer race, $1800 \mathrm{rpm}, 500 \mathrm{lbf}$ 
Figure 5.25 illustrates the ACUT demodulated signal spectra. The enveloping procedure was performed using a band pass filter centered at $3 \mathrm{kHz}$. The healthy bearing (Figure 5.25(a)), as expected, does not show any sign of the defect whereas the resulting demodulated signals spectra (Figure 5.25(b), 5.25(c) and 5.25(d)) clearly show the defect frequency and its second harmonic for faults of $0.2,0.5$, and $1 \mathrm{~mm}$ wide, respectively. Similarly to the accelerometer spectra, the dominant peaks of the ACUT spectra are located around 194Hz. Similar trends were observed at different operating test conditions. It is also worth mentioning that the amplitude of the defect frequency increases with the defect size.
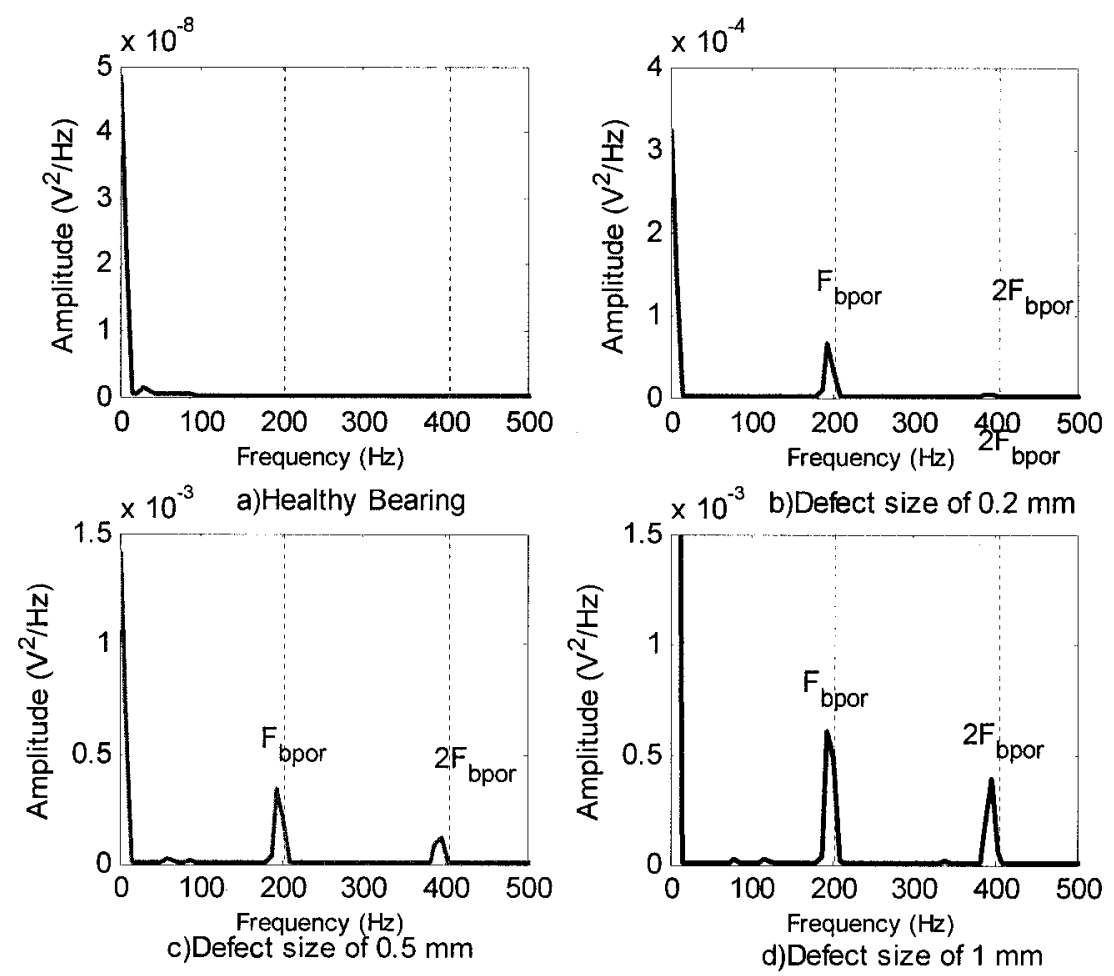

Figure 5.25 ACUT demodulated signal spectra of outer race, 1800rpm, 500lbf

Figure 5.26 illustrates the spectra of the demodulated signals collected with the Eddy current probe for healthy and defective bearing with the defect sizes of 0.2 and $0.5 \mathrm{~mm}$ and for a speed of $1800 \mathrm{rpm}$ and a load of $500 \mathrm{lbf}$. Performing the envelope analysis using a high-pass filter with a cut off frequency of $12 \mathrm{kHz}$ 
provided interesting results as shown in Figure 5.26 (a) and (b), where the fault

frequency was clearly identified with the Eddy current sensor. However, for the same test conditions and with larger defects, no trace of the fault frequency was observed.

It is noticeable to mention that the Eddy current probe was set to be in a range of maximum $1 \mathrm{~mm}$ distance from the bearing housing. One reason, which might have affected the accuracy of the reading of the Eddy current sensor, was the manual reinstallation of the sensor each time the test bearing was changed. Dadouche et al (2007) have also reported the shortcoming of the Eddy current sensor in their experiments. However, the eddy current sensor showed its capability of detecting different faults types on the bearing components for variable operating conditions.
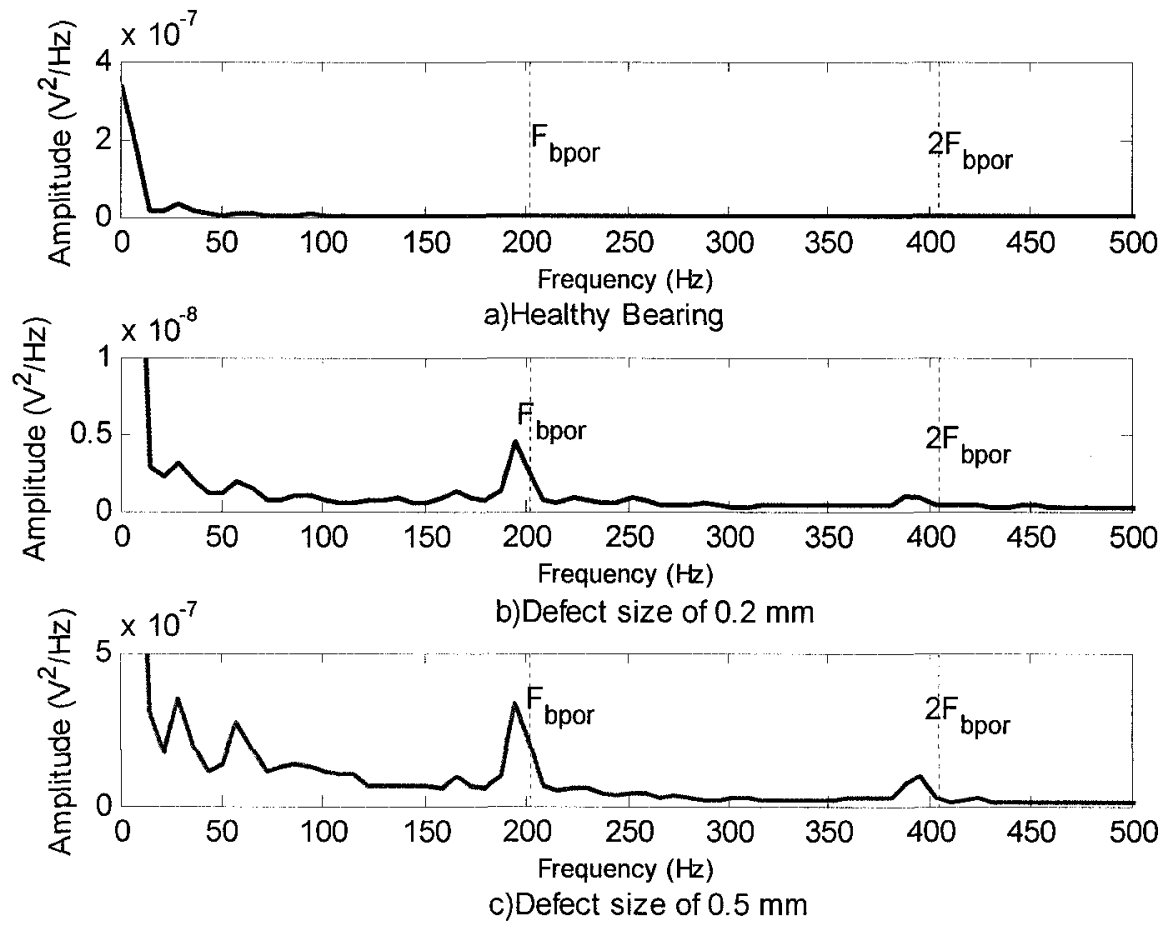

Figure 5.26 Eddy current demodulated signal spectra of outer race,

$1800 \mathrm{rpm}, 500 \mathrm{lbf}$

The strain gage sensor was evaluated only with a defective outer race with defect sizes of $0.2,0.5$, and $1.5 \mathrm{~mm}$. The radial accelerometer was also used for comparison purposes. Figure 6.27 shows raw time signal obtained with the strain 
gage, its PSD, and then the PSD of the enveloped signal. After demodulating the filtered signal and estimating its PSD, a peak at the frequency of $195 \mathrm{~Hz}$ was clearly identified as shown in Figure 6.27(c). According to Table (3-4), the first resonant frequency of the attached beam was $168.3 \mathrm{~Hz}$, nearly $40 \mathrm{~Hz}$ smaller than the theoretical defect frequency. Based on the different signals obtained with the other sensors, the system components do not have any natural frequency or excitation source close to the measured peak at $195 \mathrm{~Hz}$. Therefore, and since the defect frequency is too close to the measured one, the dominant frequency of $195 \mathrm{~Hz}$ presented in the spectrum could considered the defect frequency. The combination of the considered beam and the strain gage could not detect any defect smaller than $1.5 \mathrm{~mm}$.

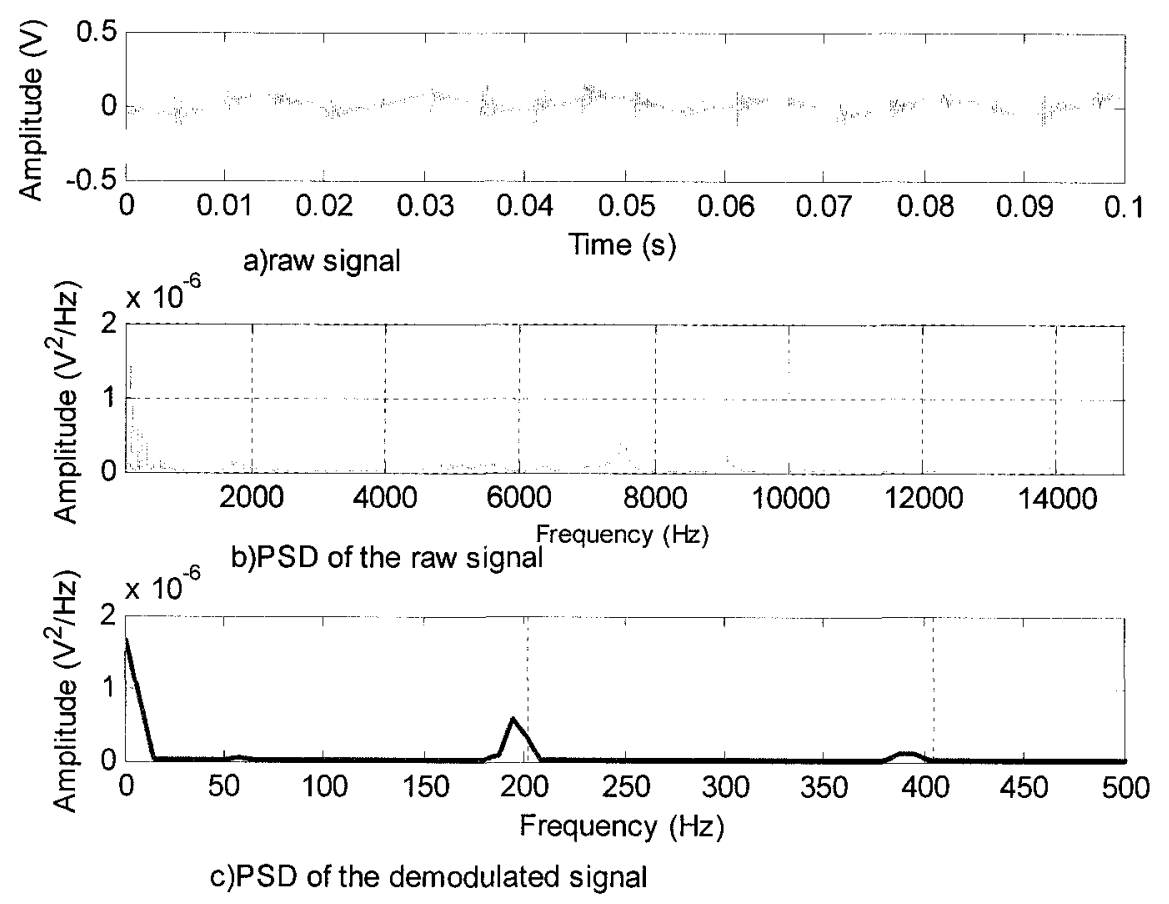

Figure 5.27 Strain gage, defective outer race, 1800rpm, 500lbf, defect size of $1.5 \mathrm{~mm}$ 


\subsubsection{Time-Frequency Analysis}

\subsubsection{Mother Wavelet Selection}

There are many available functions that can be used as a mother wavelet for CWT analysis of a defective bearing (e.g. Daubechies, Haar, Mayer, Morlet, etc) (Cohen, 1995). As defined in equation 4-21 (Chapter 4), the wavelet transform is the inner product of the signal and the complex conjugate of $\psi_{\tau, S}(t)$. Therefore, the wavelet analysis results in wavelet coefficients indicating the closeness of the signal to the mother wavelet. It has been shown in Figure 5.5 and 5.6 that the vibration and acoustic signals of a defective outer race represent periodic sinusoids decaying exponentially overtime.

The $7^{\text {th }}$ derivative of the complex Gaussian function was selected as the basic wavelet by empirical trial and error search process used to extract the characteristic of the vibration signals from the rolling element bearing. Complex Gaussian wavelets, which represent the "family" of wavelets based on the complex Gaussian function $\mathrm{g}(t)$, are used to interpret experimental data by taking the $n$ derivative of $f(t)$.

$$
g(t)=A_{n} \exp \left(-t^{2}-i t\right)
$$

The integer $n$ is the parameter of this family and in the previous formula, $A_{n}$ is such that

$\left\|g^{(n)}\right\|=1$, where $g^{(n)}$ is the $n^{t h}$ derivative of $g(t)$. Figure 5.28 shows the real part, imaginary part, the modulus and the phase angle of the selected mother wavelet. 


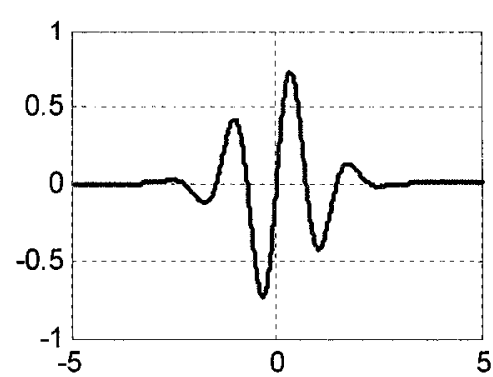

a) Real part

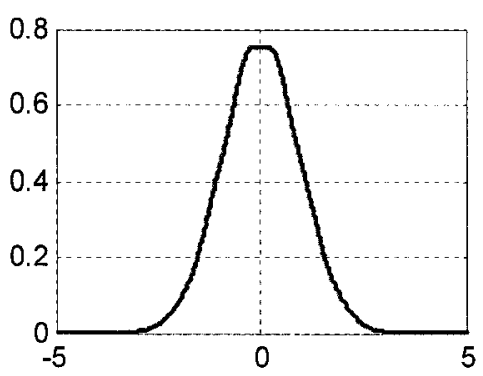

c) Complex modulus.

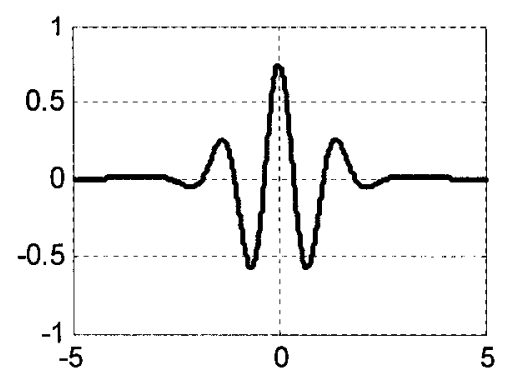

b) Imaginary part

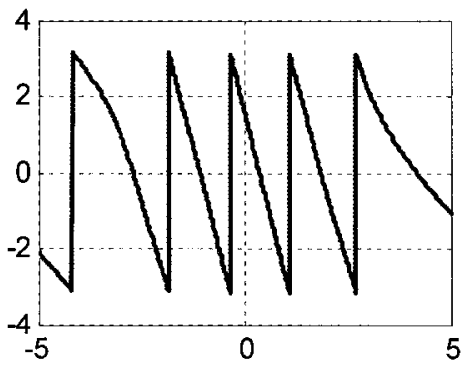

d) Phase angle.

Figure 5.28 The $7^{\text {th }}$ derivative of the complex Gaussian function characteristics

\subsubsection{Application}

Using the $7^{\text {th }}$ derivative of the complex Gaussian function as the mother wavelet, WT was applied to the radial vibration data for variable test conditions and for a defect on the outer race of $0.2 \mathrm{~mm}$ wide (Figure 5.29). As shown in this Figure, the major wavelet coefficients are apart from each other by $0.01 \mathrm{~s}$ for a speed of $900 \mathrm{rpm}, 0.005 \mathrm{~s}$ for $1800 \mathrm{rpm}$, and $0.004 \mathrm{~s}$ for $2100 \mathrm{rpm}$. Actually, these time intervals correspond to the outer race defect frequency of the bearing (Table 3-2). The initial identifying feature on the time-frequency distribution is the excited resonances. For most of the cases, the excited resonance frequencies are in a range between $1200 \mathrm{~Hz}$ to $3500 \mathrm{~Hz}$ and are very close to each other over all speed and load conditions as they were identified in the spectrum analysis. The increase of the shaft speed and load slightly affected the resonance frequencies of the defective bearing system. However, the presence of background noise while increasing the shaft speed, in the time-frequency diagrams (Figure 5.29), is clearly 
shown. Considering Figure 5.29(a), (b), and (c), the influence of the load on the radial vibration is not very significant. By increasing the load, the impulse response of the bearing due to the defect impact comprises a progressive decay with time. This is more visible in the same Figures, once the motor speed increases.

The analysis of axial vibration signatures for different operating bearing conditions using the same mother wavelet produced similar results to those of radial vibration.

The time-frequency comparison of acoustic signatures (ACUT) for a defect size of $0.2 \mathrm{~mm}$ under variable test conditions is presented in Figure 5.30. Evidence of defects can be identified in these plots. However, the interfering cross terms, especially for the low shaft speed conditions make it difficult to identify the exact time of defect occurrence as well as the region of excitation of the resonances.

The wavelet analysis on Eddy current probe and PUT sensors did not produce any useful information. 


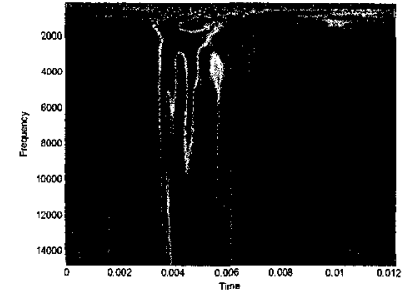

a) $900 \mathrm{rpm}, 100 \mathrm{lbf}$

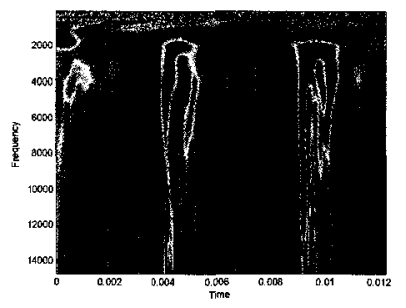

d) $1800 \mathrm{rpm}, 100 \mathrm{lbf}$

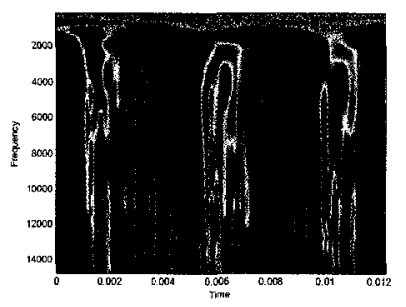

g) 2100rpm, 100lbf

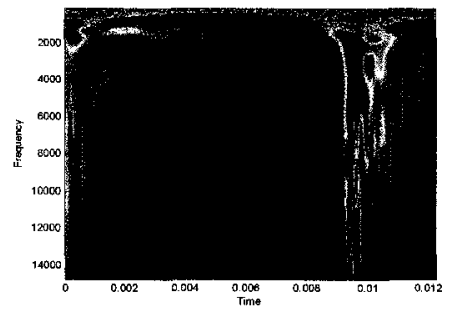

b) $900 \mathrm{rpm}, 250 \mathrm{lbf}$

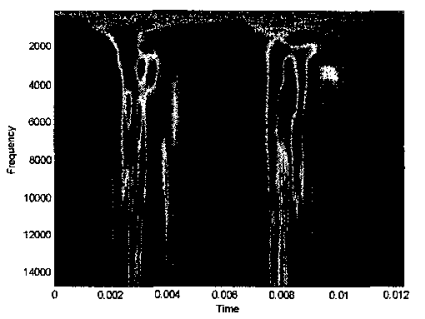

e) $1800 \mathrm{rpm}, 2501 \mathrm{bf}$

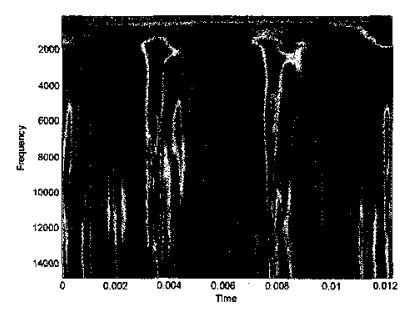

h) $2100 \mathrm{rpm}, 250 \mathrm{lbf}$

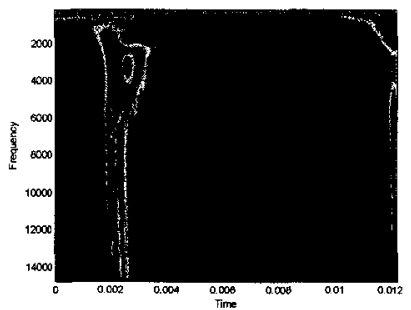

c) $900 \mathrm{rpm}, 500 \mathrm{lbf}$

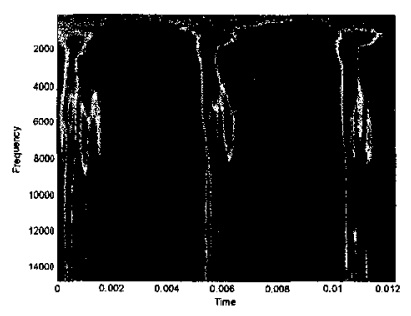

f) $1800 \mathrm{rpm}, 5001 \mathrm{bf}$

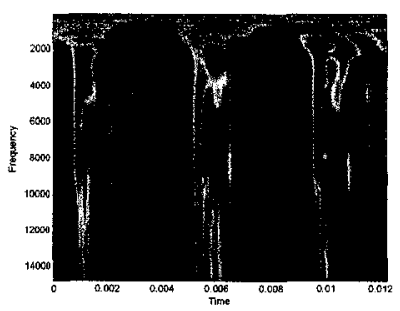

i) $2100 \mathrm{rpm}, 500 \mathrm{lbf}$

Figure 5.29 Wavelet analysis of defective outer race, radial accelerometer, defect width of $0.2 \mathrm{~mm}$ 


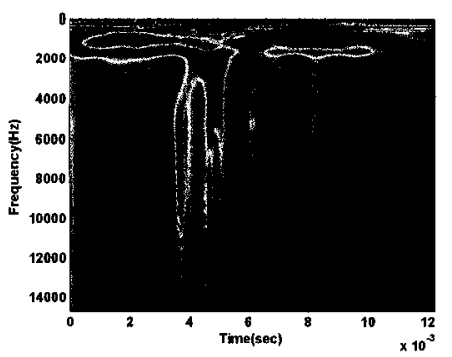

a) $900 \mathrm{rpm}, 100 \mathrm{lbf}$

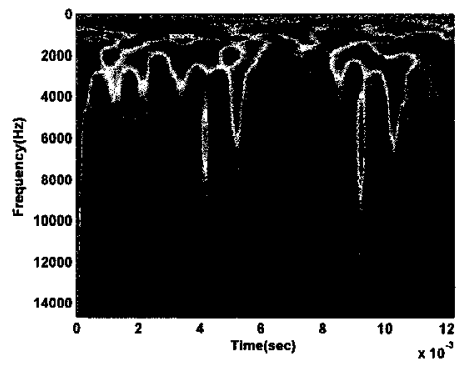

d) $1800 \mathrm{rpm}, 1001 \mathrm{bf}$

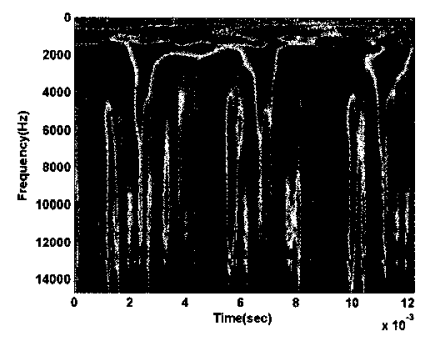

g) $2100 \mathrm{rpm}, 100 \mathrm{lbf}$

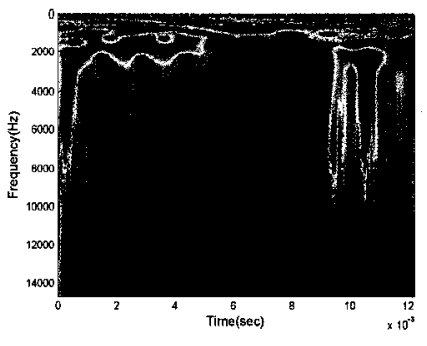

b) $900 \mathrm{rpm}, 250 \mathrm{lbf}$

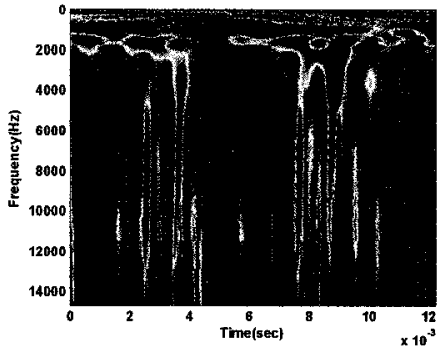

e) $1800 \mathrm{rpm}, 250 \mathrm{lbf}$

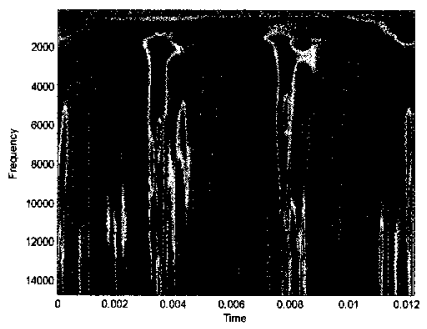

h) $2100 \mathrm{rpm}, 250 \mathrm{lbf}$
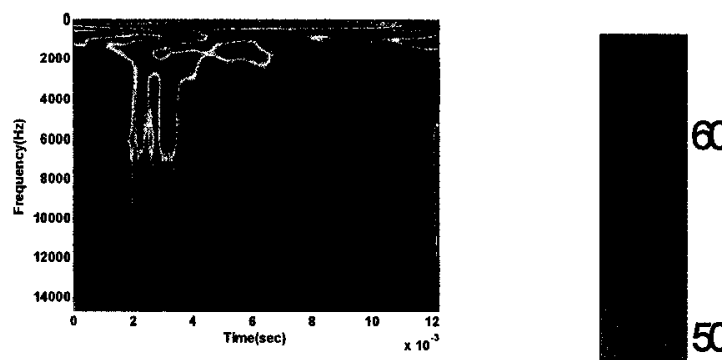

c) $900 \mathrm{rpm}, 500 \mathrm{lbf}$

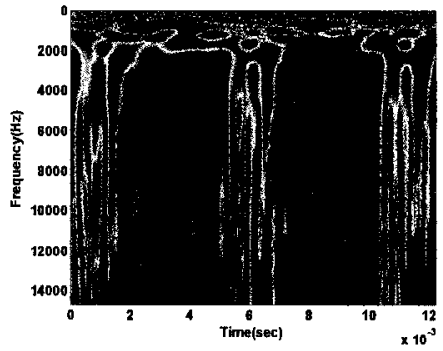

f) $1800 \mathrm{rpm}, 500 \mathrm{lbf}$

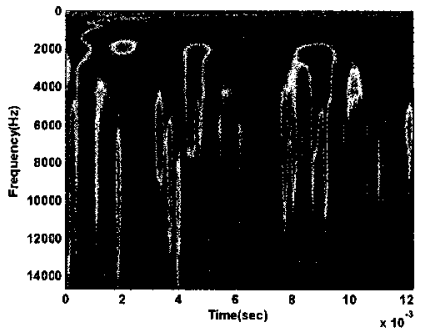

i) $2100 \mathrm{rpm}, 500 \mathrm{lbf}$

Figure 5.30 Wavelet analysis of defective outer race, ACUT,

defect width of $0.2 \mathrm{~mm}$ 


\subsubsection{Envelope Spectrum of Wavelet Transform}

Figures 2.30 to 2.33 show the three-dimensional joint scale-frequency distributions of the radial vibration signatures for normal and defective bearings for defect width of $0.2,0.5$, and $1 \mathrm{~mm}$ under a motor speed of $1800 \mathrm{rpm}$ and a load of $500 \mathrm{lbf}$.

The driving motor speed is visible in Figure 5.31 and its second harmonic is also visible in Figures 5.32, 5.33, and 5.34. In the faulty cases, the outer race defect frequency components at $201 \mathrm{~Hz}$ and its harmonic at $400 \mathrm{~Hz}$ are also identified in the distributions. The wavelet-based envelope spectrum analysis of other test conditions (speeds and loads) and defect width sizes show less and more similar results to those presented in Figures 5.32 to 5.34. The analysis of axial vibration signatures between healthy and defective bearings using the envelope spectra of wavelet transform illustrates similar results of those of radial vibrations. It is worth mentioning that by increasing the defect size, the amplitude of $f_{b p o r}$ increases. The amplitude of $f_{\text {bpor }}$ is $4.002 \times 10^{-6} \mathrm{~V}$ for a healthy bearing, $0.1829 \mathrm{~V}$ for $0.2 \mathrm{~mm}$ faulty bearing, $0.618 \mathrm{~V}$ for $0.5 \mathrm{~mm}$ faulty bearing and $5.168 \mathrm{~V}$ for $1 \mathrm{~mm}$ faulty bearing, respectively. Therefore, the increase of the wavelet-based spectra amplitude is a good indicator for bearing deteriorations.

The time-frequency comparison of the acoustic signatures obtained by the ACUT for normal and faulty bearings with a defect size of $0.2 \mathrm{~mm}$ on its outer race is illustrated in Figures 5.35 and 5.36. In the case of healthy bearing (Fig. 5.35), a peak of the second harmonics of shaft speed $(60 \mathrm{~Hz})$ is clearly visible and as expected, $f_{\text {bpor }}$ components cannot be seen. In Figure 5.36, a dominant peak at $f_{\text {bpor }}$ component is observed, which identifies the faulty bearing. Furthermore, the second harmonic of the driving frequency is also visible in the defective bearing distribution. 


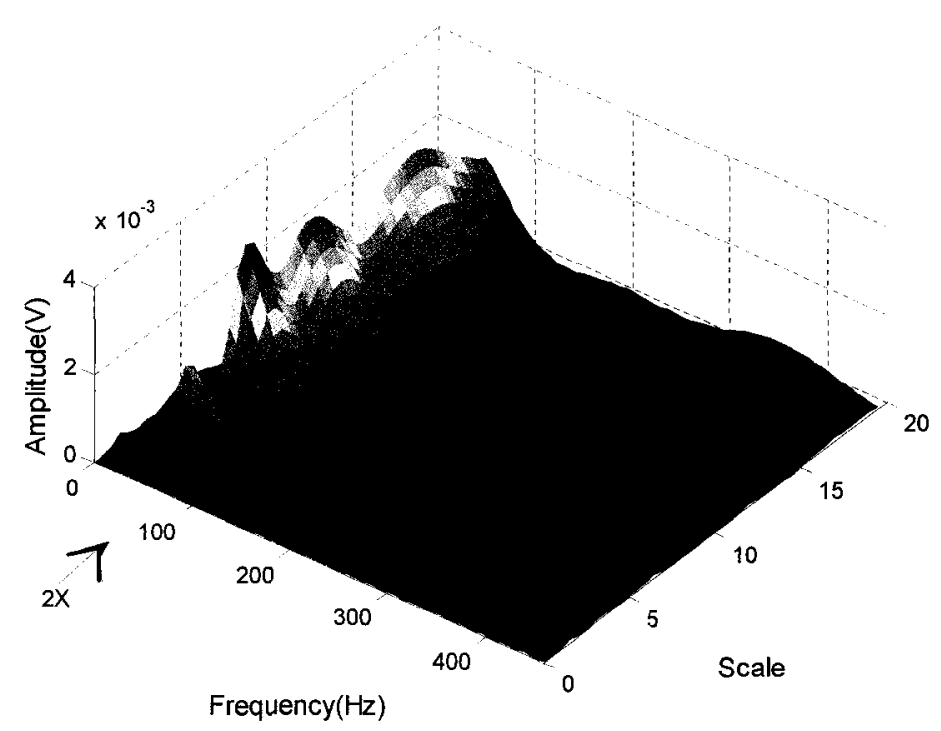

Figure 5.31 Wavelet-based envelop spectrum of healthy bearing, $1800 \mathrm{rpm}, 500 \mathrm{lbf}$, radial vibration

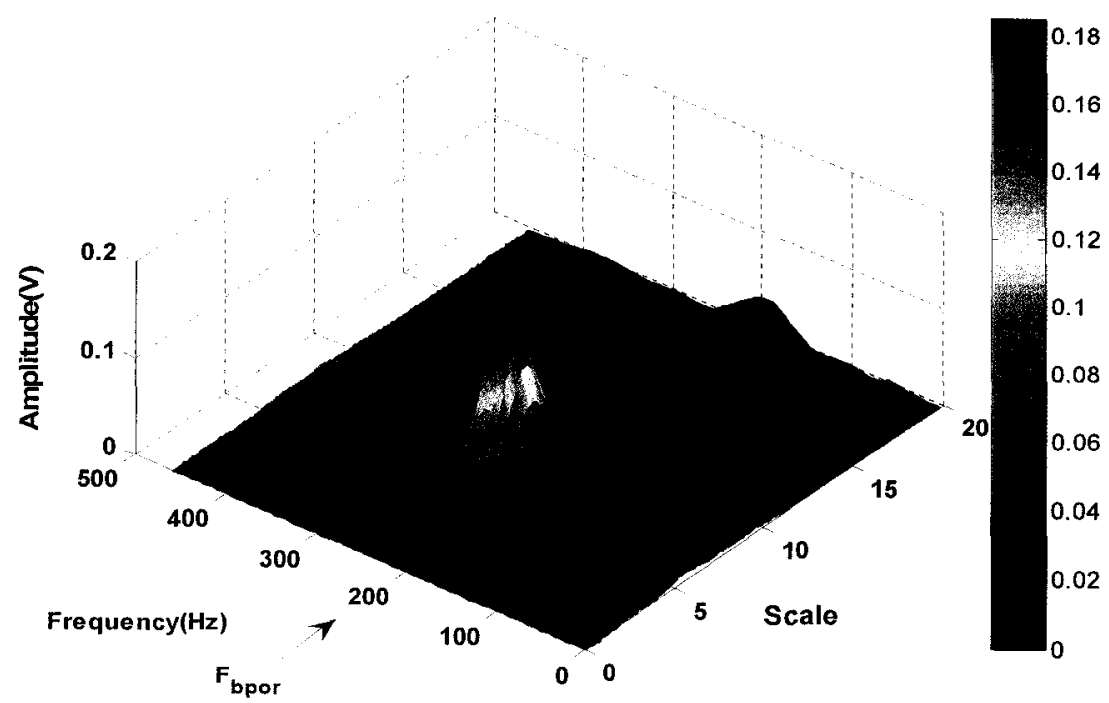

Figure 5.32 Wavelet-based envelop spectrum of defective outer race, 1800rpm $500 \mathrm{lbf}$, defect width of $0.2 \mathrm{~mm}$ 


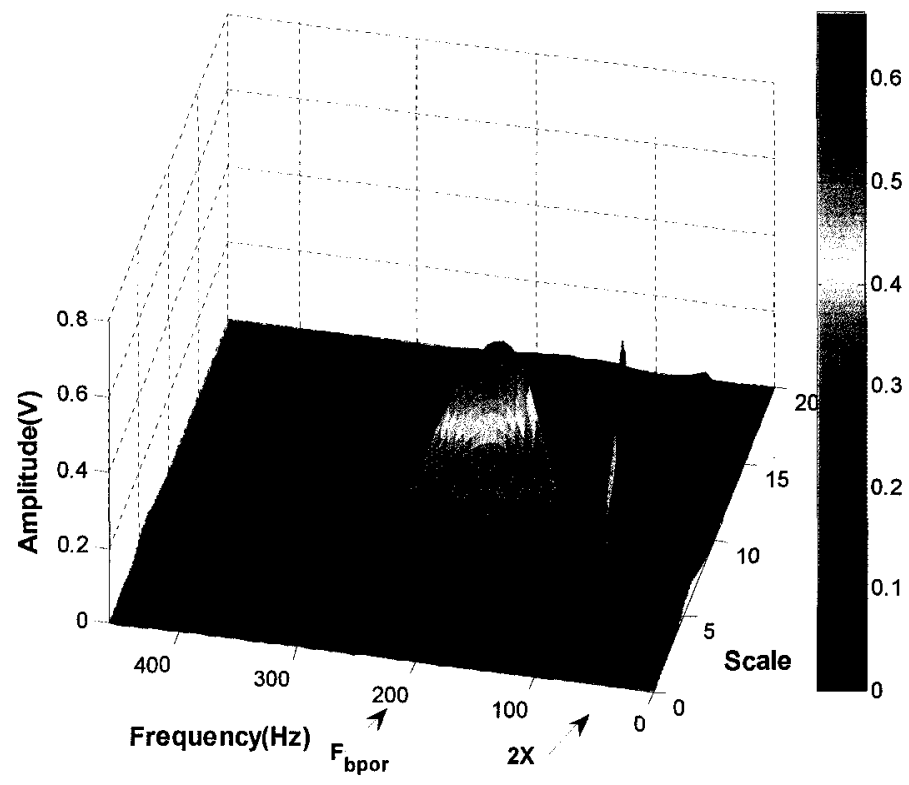

Figure 5.33 Wavelet-based envelope spectrum of defective outer race, $1800 \mathrm{rpm}$, 500lbf, radial vibration, defect width of $0.5 \mathrm{~mm}$

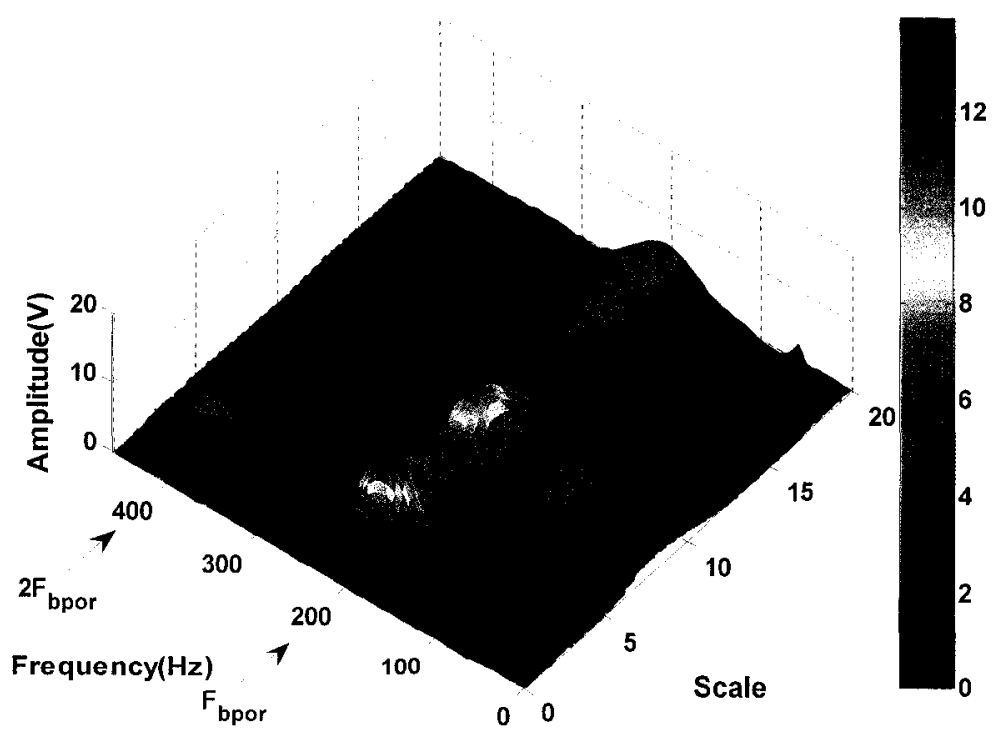

Figure 5.34 Wavelet-based envelop spectrum of defective outer race, $1800 \mathrm{rpm}, 500 \mathrm{lbf}$, radial vibration, defect width of $1 \mathrm{~mm}$ 


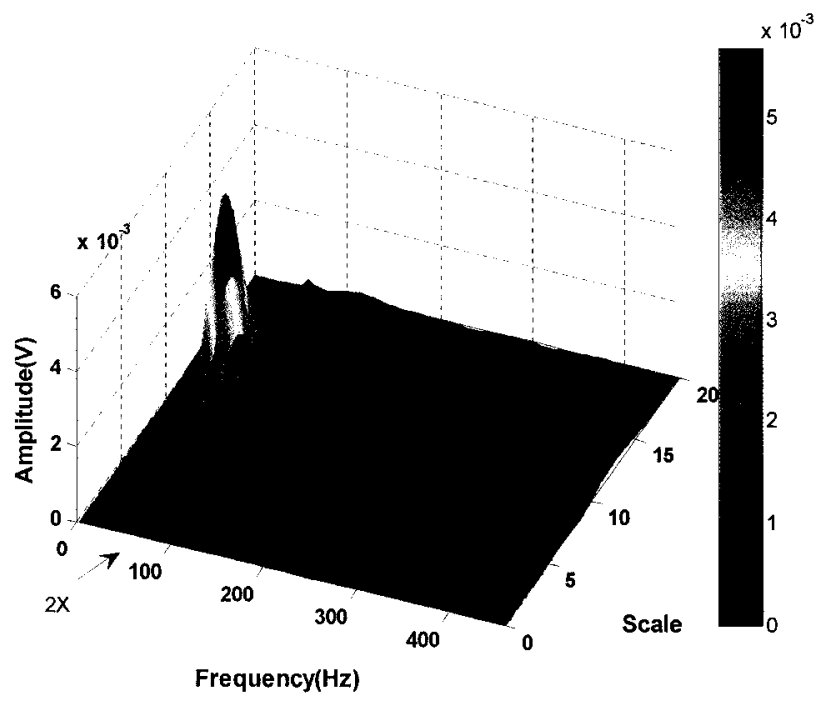

Figure 5.35 Wavelet-based envelope spectrum of healthy bearing, $1800 \mathrm{rpm}$, 500lbf, acoustic signature (ACUT)

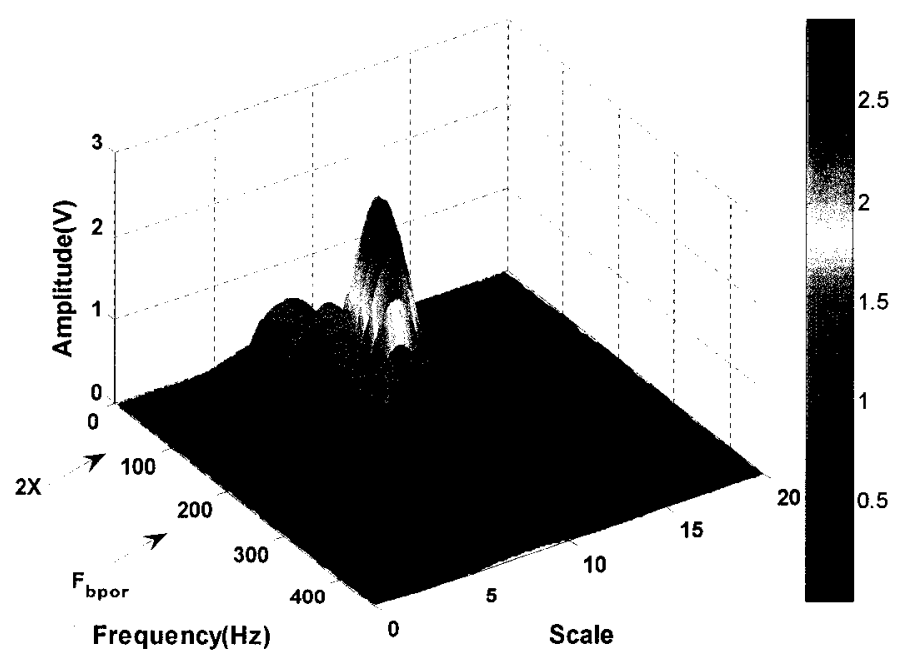

Figure 5.36 Wavelet-based envelope spectrum of defective outer race, $1800 \mathrm{rpm}, 500 \mathrm{lbf}$, acoustic signature (ACUT), defect width of $1 \mathrm{~mm}$ 


\subsection{Defective Inner Race Analysis}

Due to the huge number of data files recorded during the experiments, only selected cases will be discussed and analyzed in this Section. Considering a constant speed of 1800rpm and a load of 5001bf, Figures 5.37 and 5.38 show the time history signals of healthy and defected bearings collected with the radial accelerometer and the ACUT. The fault sizes ranges from 0.2 to $1 \mathrm{~mm}$ and were localized on the bearing inner race. As could be seen on the figures, the ACUT signatures (Figure 5.38) contain more background noise than those of the accelerometer.
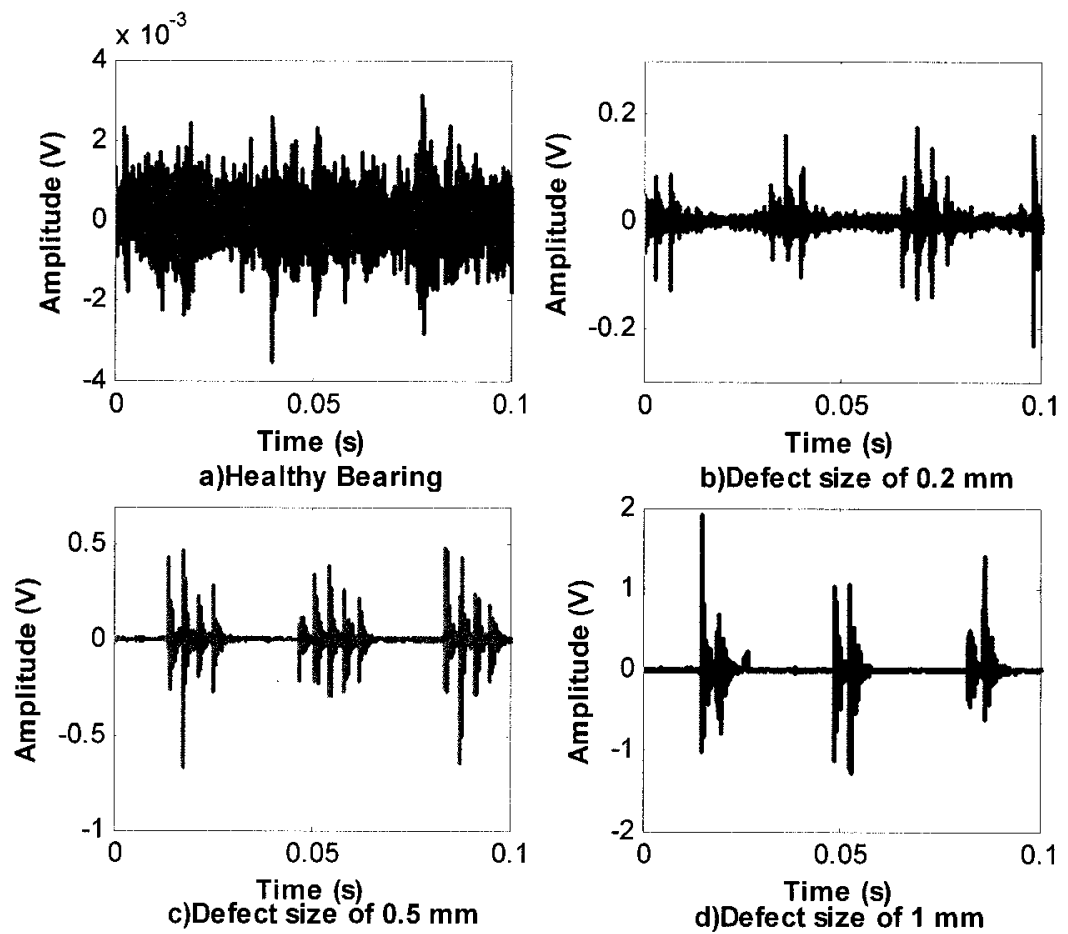

Figure 5.37 Accelerometer time history signal of inner race, 1800rpm, 500lbf 

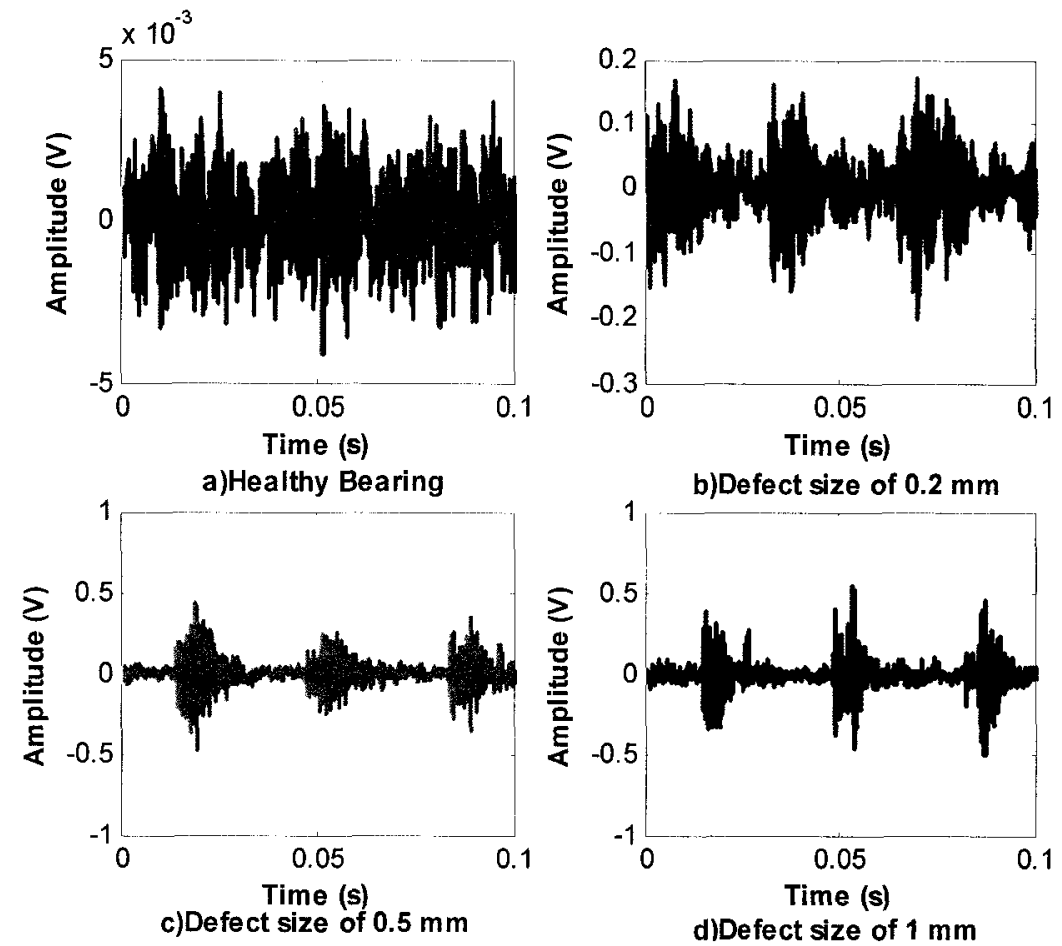

Figure 5.38 ACUT time history signal of inner race, 1800rpm, 500lbf

\subsubsection{Time-Domain Analysis}

The sensitivity of the time-domain parameters to the defect size is analyzed by defining detectability factors, which represent the ratio of the time metrics of the defective bearing to those of the healthy bearing. Figure 5.39 shows the evolution of these factors, extracted from the vibration signals, versus the defect size. The horizontal line represents the healthy bearing condition. As Figure 5.39 shows, the peak and RMS defect detectability factors represent the best indicators of fault detection and progression, followed by the kurtosis factor. The crest factor is a good detector for defect growth up to a size $1 \mathrm{~mm}$ only. This observation was also confirmed by other test conditions. However, all the detectability parameters exhibit, individually, a significant increase in comparison with the healthy bearing condition.

Figure 5.40 illustrates the detectability factors, obtained from the ACUT acoustic 
signatures, against the inner race defect size. Although all the time metrics associated with each defect size were larger than the healthy bearing condition case, the crest factor and kurtosis were poor indicators of defect growth. Similarly to the vibration signature, the peak and RMS detectbility factors were more reliable.

Time-domain analysis was also performed on Eddy current and PUT signals, but no significant results were obtained.

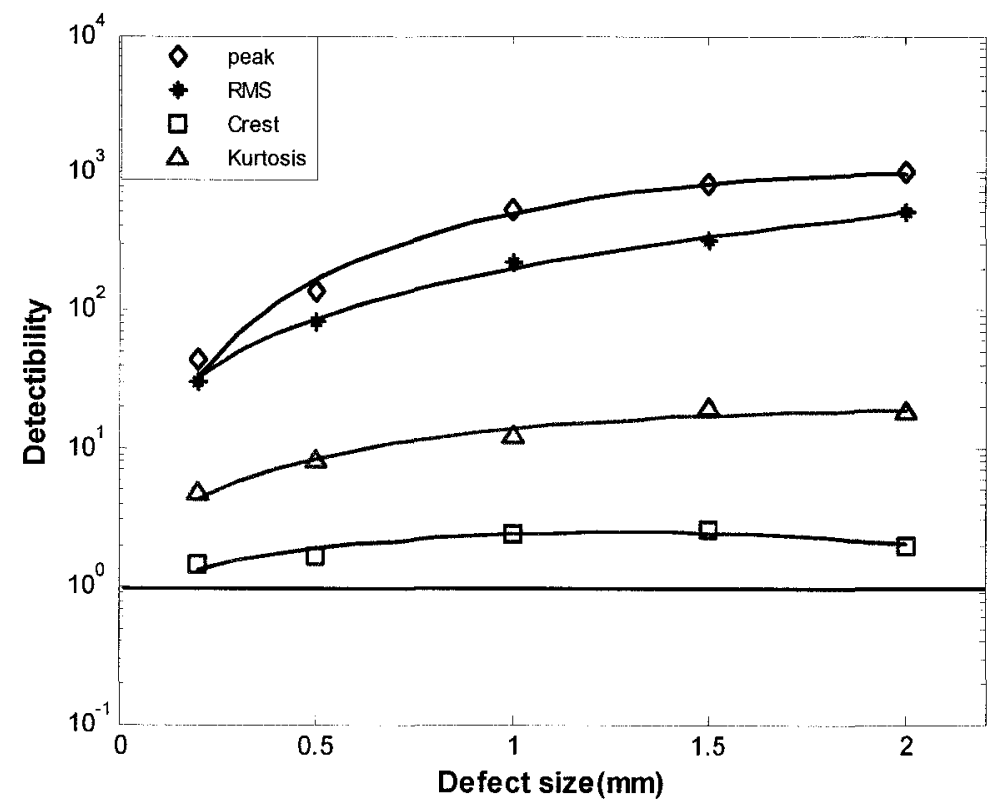

Figure 5.39 Defect detectability factors versus defect size, defective inner race, 1800rpm, 500lbf, vibration signature 


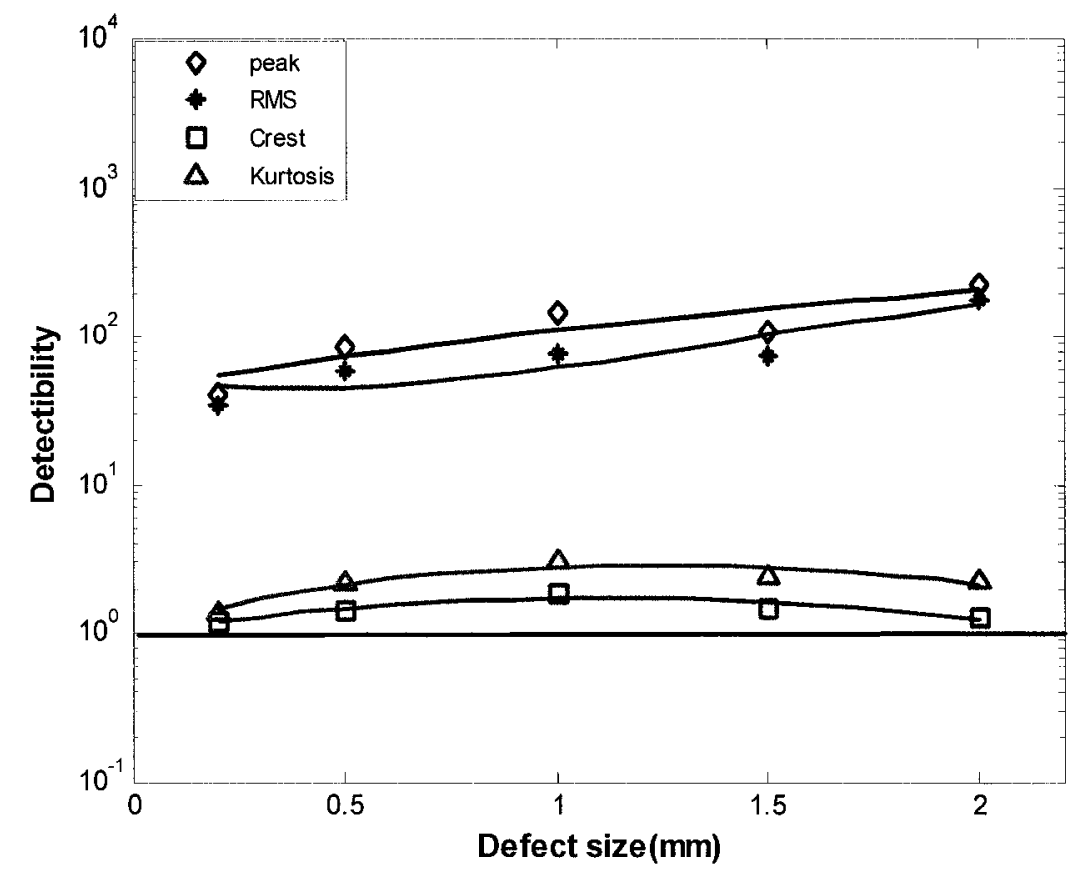

Figure 5.40 Defect detectability factors versus defect size, defective inner race, $1800 \mathrm{rpm}, 500 \mathrm{lbf}$, acoustic signature (ACUT)

\subsubsection{Frequency-Domain Analysis}

Figure 5.41 and 5.42 show the spectra of the accelerometer and ACUT for the cases of healthy and defective inner races for a constant speed of $1800 \mathrm{rpm}$ and a load of $500 \mathrm{lbf}$, up to the Nyquist frequency of $15 \mathrm{kHz}$. As expected for most cases, the dominant excitation frequency range was located between 1200 and $3500 \mathrm{~Hz}$. 

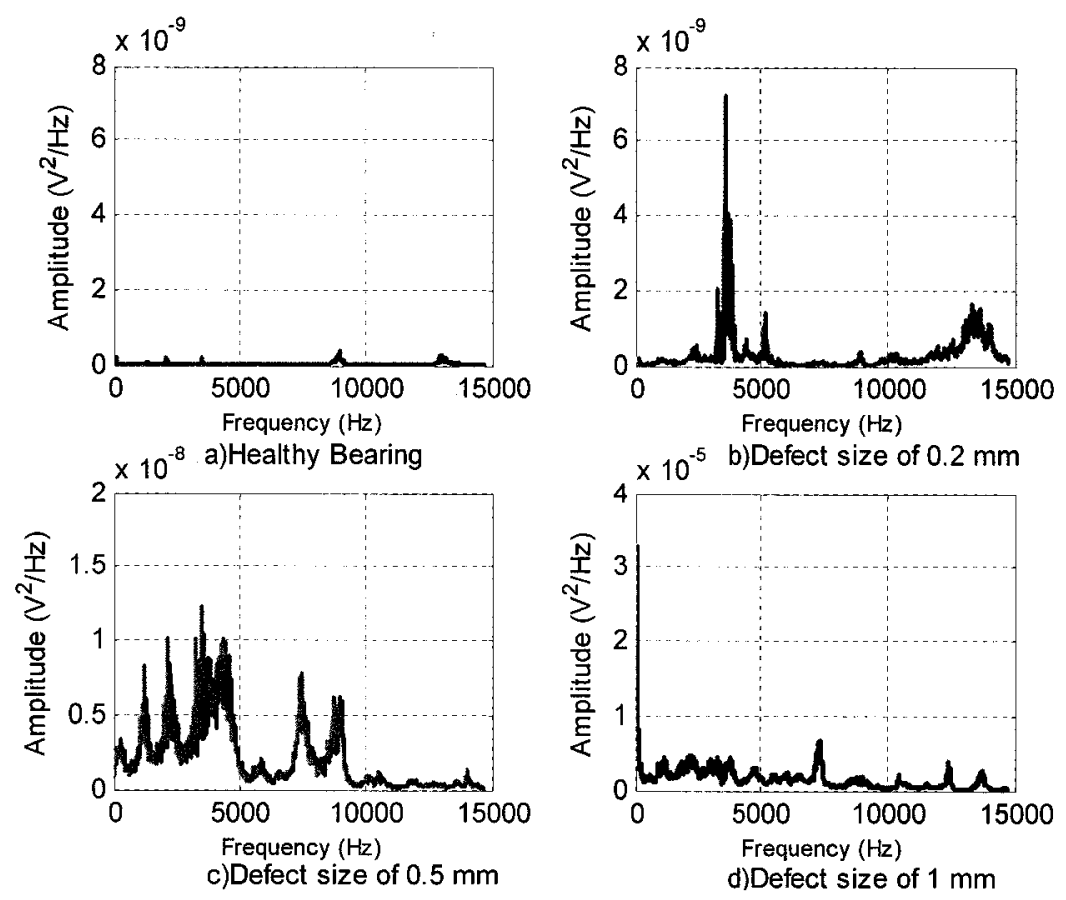

Figure 5.41 Vibration signature spectra of inner race, $1800 \mathrm{rpm}, 500 \mathrm{lbf}$
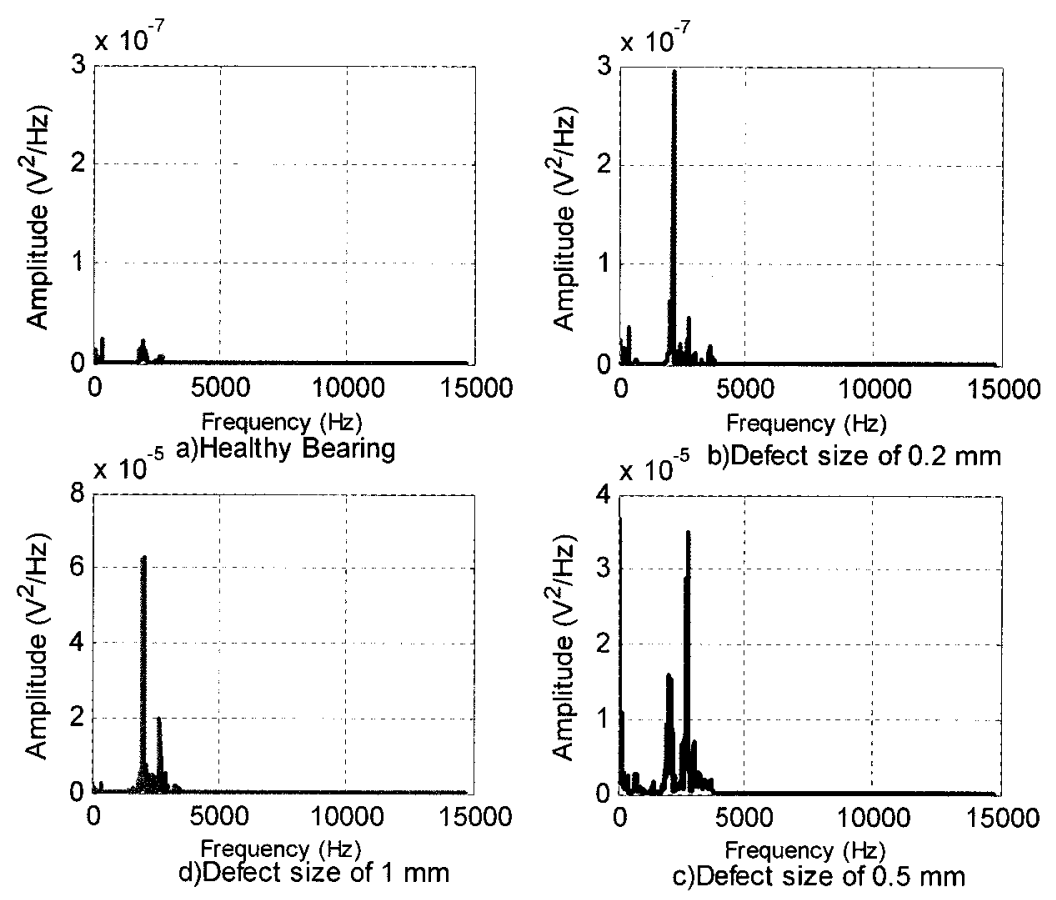

Figure 5.42 Acoustic signature (ACUT) spectra of inner race, $1800 \mathrm{rpm}, 500 \mathrm{lbf}$ 
Figure 5.43 illustrates the PUT signal spectra up to $500 \mathrm{~Hz}$. The vertical dashed lines on the plots represent the expected defect frequency location $\left(f_{b p i r}=277 \mathrm{~Hz}\right)$. Despite the interesting results obtained with defects on the outer race, the PUT signature did not give any sign of fault occurrence on the inner race. Except for the visible and dominant shaft frequency and its harmonics, the spectra do not show any trace of the defect frequency.
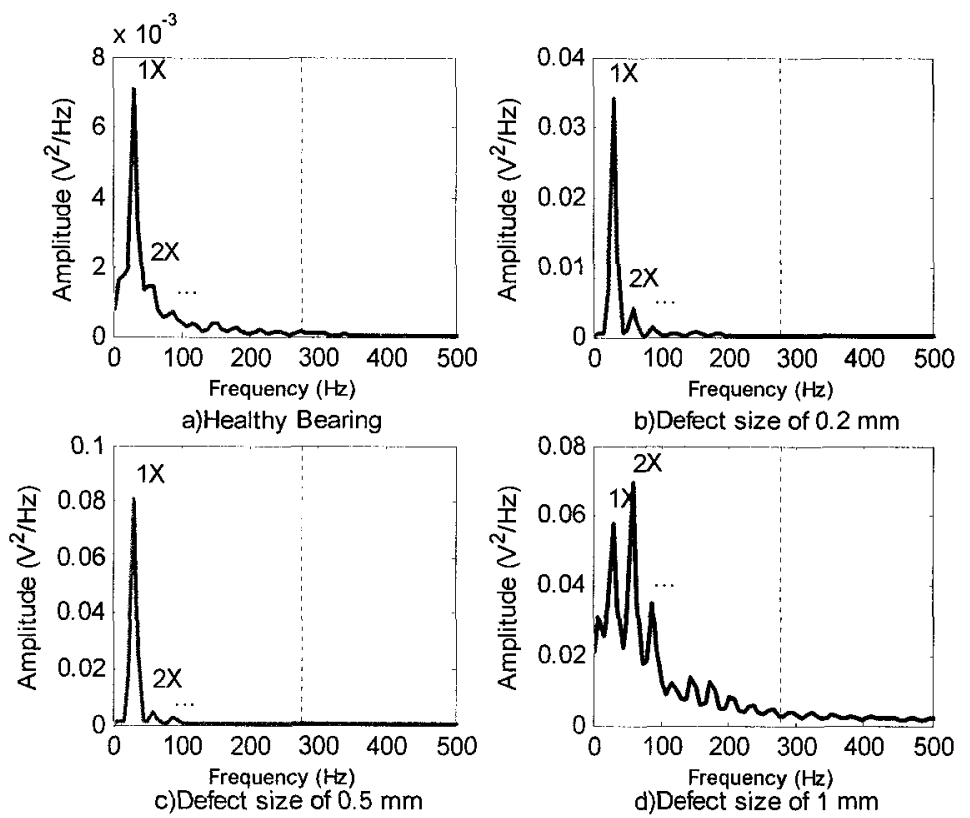

Figure 5.43 Acoustic signature (PUT) spectra of inner race, 1800rpm, $500 \mathrm{lbf}$

The HFRT procedure was applied on the time signals of the accelerometer and ACUT presented above (Figures 5.37 and 5.38) and the resulted spectra show that both the accelerometer and ACUT were able to detect the defects with different sizes (Figures 5.44 and 5.45). The shaft rotation frequency and some of its harmonics are also present in the spectra. Furthermore, side bands of shaft frequency are clustered around the characteristic fault frequency. This modulation effect results from the variation of the defect frequency amplitude due to the passage of the defect through the bearing loaded area. The defect frequency amplitude reaches its highest level when entering the loaded zone and otherwise 
decreases. This phenomenon is synchronized with the shaft rotation and results in the modulation of the defect frequency (Dadouche et al, 2007).
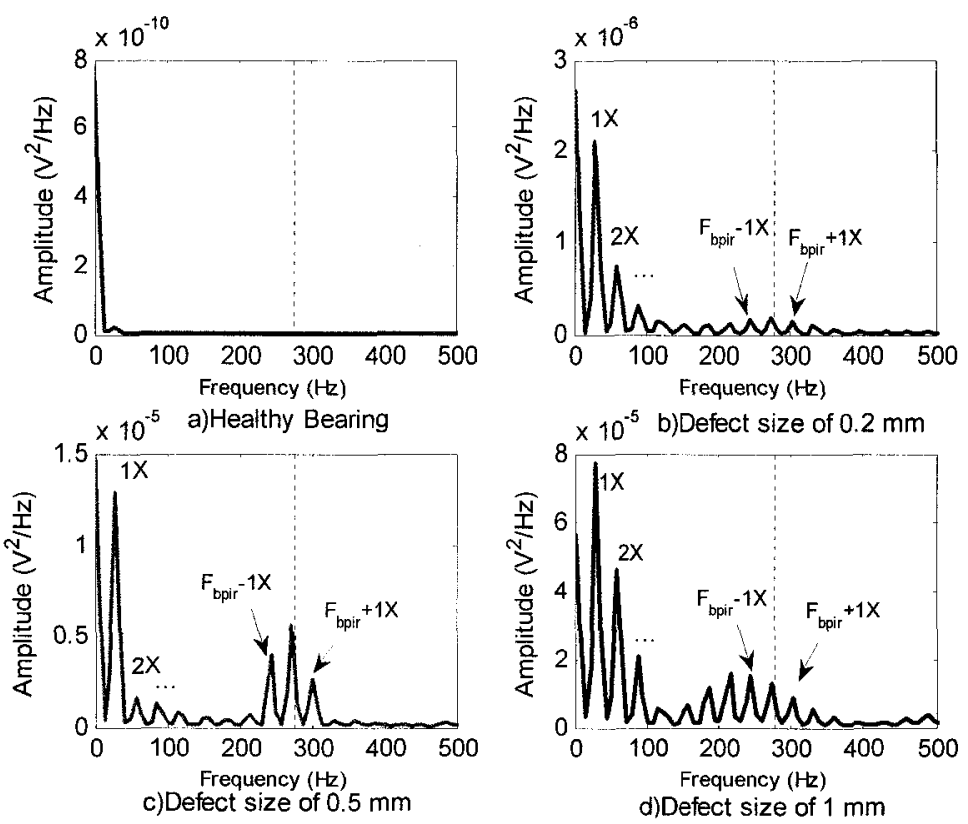

Figure 5.44 Demodulated vibration signature spectra of inner race, $1800 \mathrm{rpm}, 500 \mathrm{lbf}$
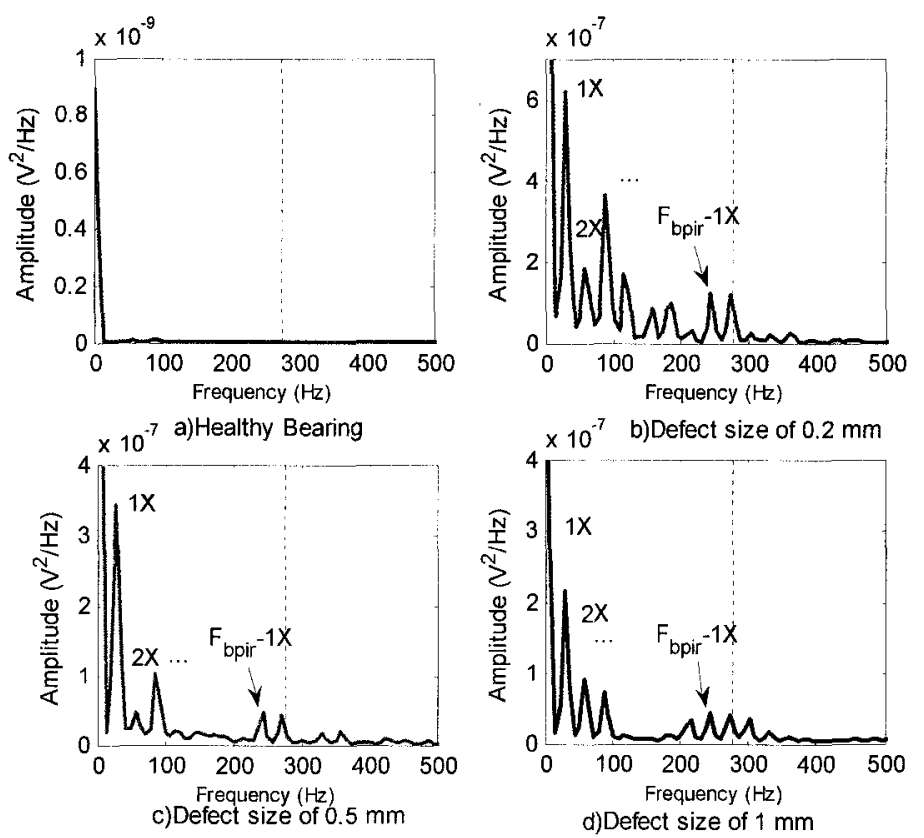

Figure 5.45 Demodulated acoustic signature (ACUT) spectra of inner race, 1800rpm, 5001bf 
Despite the case of the outer race defects, the eddy current probe did not show the defect feature for small $(0.2 \mathrm{~mm})$ and medium $(0.5 \mathrm{~mm})$ defects on the inner race. However, for a $1 \mathrm{~mm}$ wide defect, the shaft rotating frequency and a lot of its harmonics are present in the spectrum and the defect frequency was barely identified as shown in Figure 5.46.

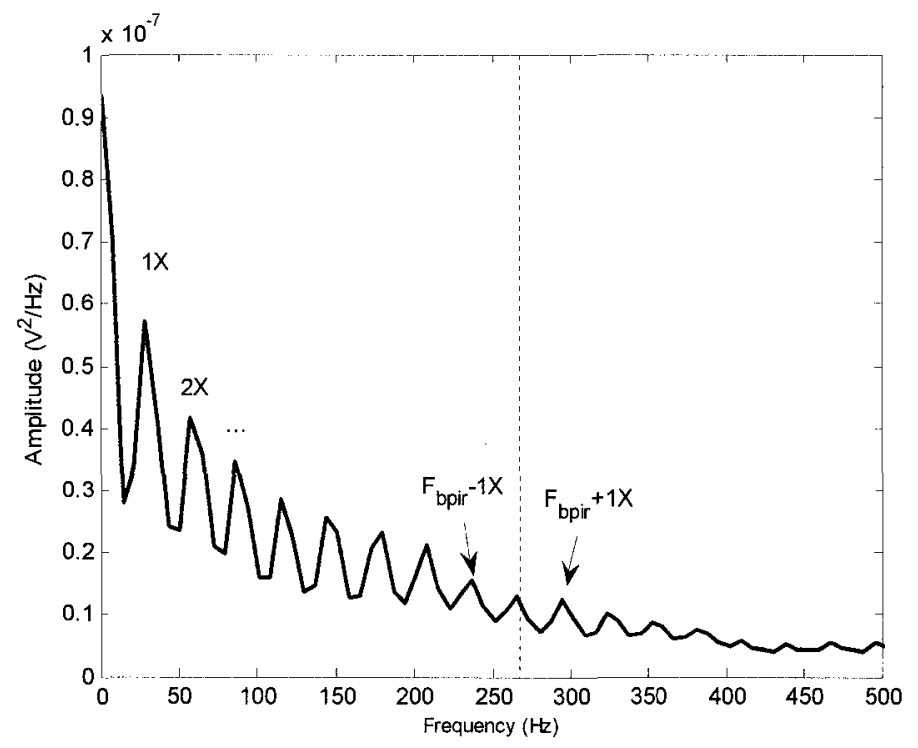

Figure 5.46 Demodulated eddy current signal spectrum of inner race, $1800 \mathrm{rpm}, 500 \mathrm{lbf}$, defect width of $1 \mathrm{~mm}$

\subsubsection{Time-Frequency Analysis}

Figure 5.47 illustrates the results of the wavelet based envelope spectrum of the acoustic signatures (ACUT), for a defect width of $0.5 \mathrm{~mm}$ localized on the inner race, using the $7^{\text {th }}$ complex Gaussian wavelet as the mother wavelet. The inner race defect frequency, $f_{\text {bpir }}$ was dominant on the distribution. In addition, the rotating speed frequency cluster around the defect frequency is also visible in Figure 5.47, which correlates well with the characteristic of identifying defects on the inner race. Similar results were obtained with different test conditions and for various defect widths. The vibration wavelet spectrum showed similar trends to 
those of the acoustic emission when identifying faulty inner race.

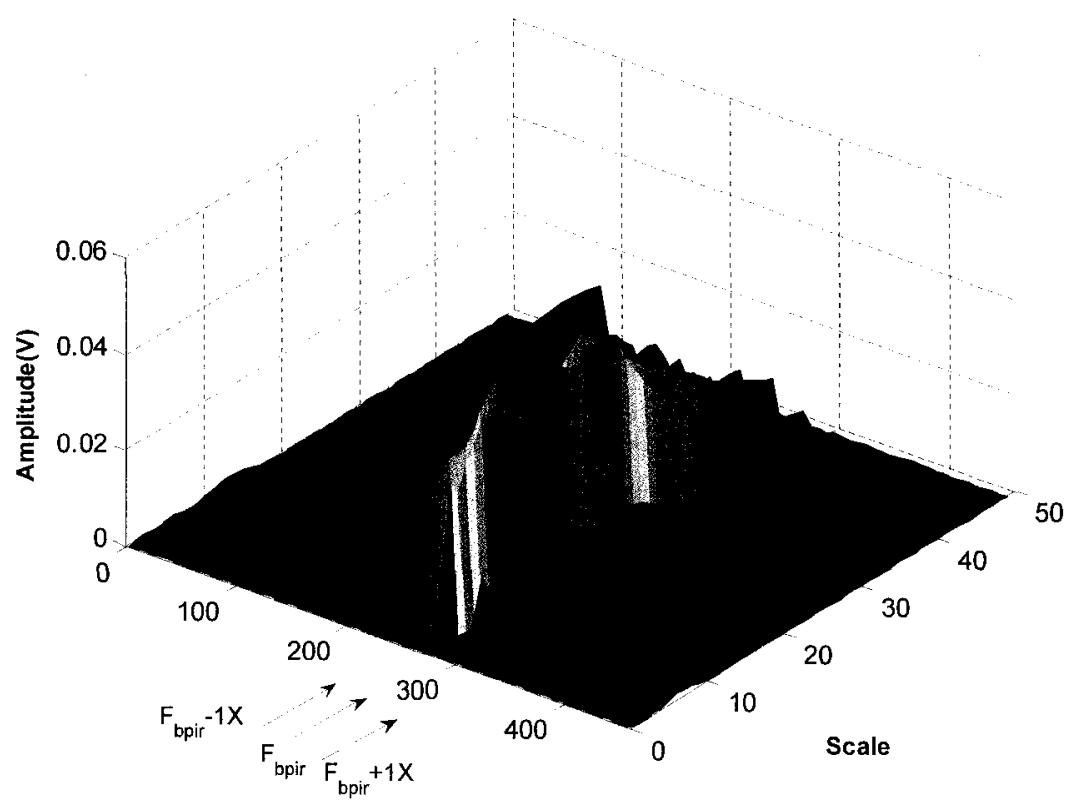

Figure 5.47 Wavelet-based envelope spectrum of defective inner race, 1800rpm, $500 \mathrm{lbf}$, acoustic signature, defect width of $0.5 \mathrm{~mm}$

\subsection{Defective Roller Analysis}

Figures 5.48 and 5.49 represent the raw signals of the accelerometer and ACUT of healthy and defective bearings, respectively. The spalls are localized on the roller and are $0.2,0.5$ and $1 \mathrm{~mm}$ wide. 

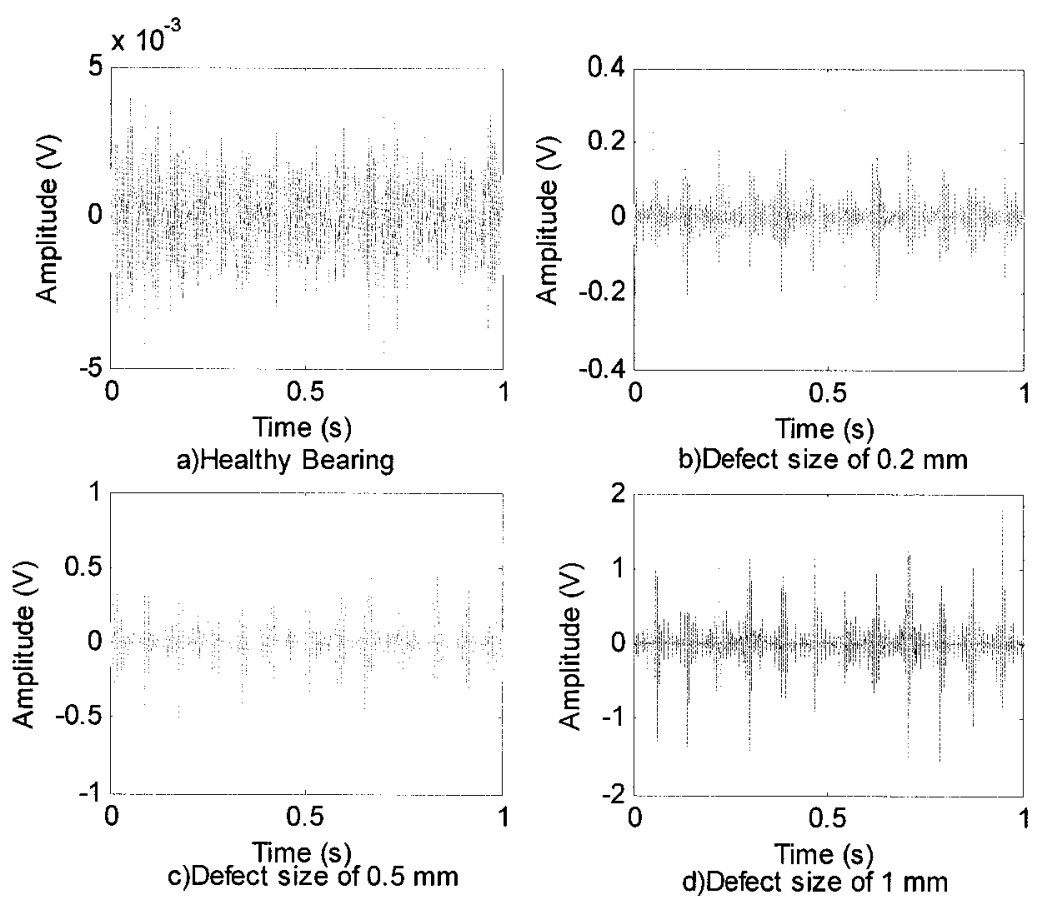

Figure 5.48 Accelerometer time history of roller, $1800 \mathrm{rpm}, 500 \mathrm{lbf}$
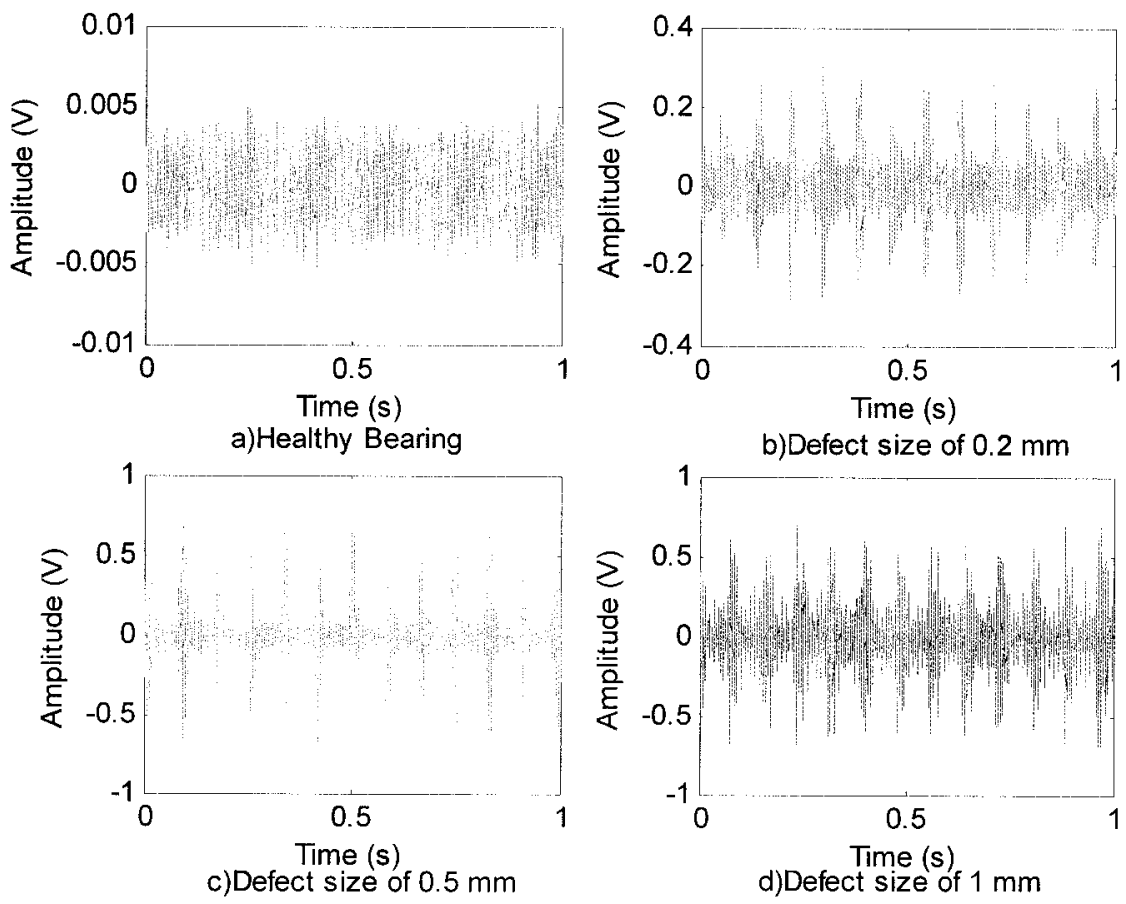

Figure 5.49 ACUT time history of roller, 1800rpm, 500lbf 


\subsubsection{Time-Domain Analysis}

The same detectability parameters were defined for the peak value, RMS, crest and kurtosis factors in the case of a defect on the roller. Figures 5.50 and 5.51 show the evolution of these parameters with the defect size for the vibration and acoustic signatures, respectively. For the vibration signal and up to a defect size of $1.5 \mathrm{~mm}$ (Figure 5.50), the peak and RMS values correlate well with defect growth. Larger defects result in a drastic increase of background noise affecting the detectability parameters. However, the defect detectability of kurtosis shows the best results.

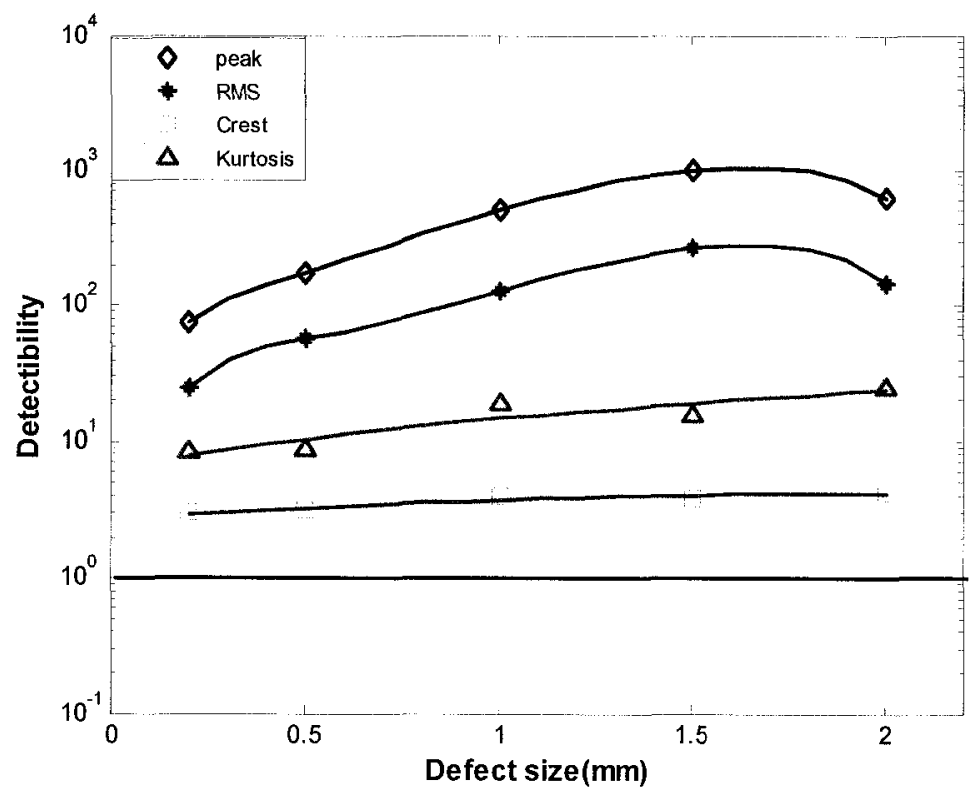

Figure 5.50 Defect detectability, vibration signature, roller, $1800 \mathrm{rpm}, 500 \mathrm{lbf}$

In Figure 5.51, the ACUT detectability parameters show a higher level than those of healthy bearing; whereas, none of them show a relevant trend in respect with the defect size. 


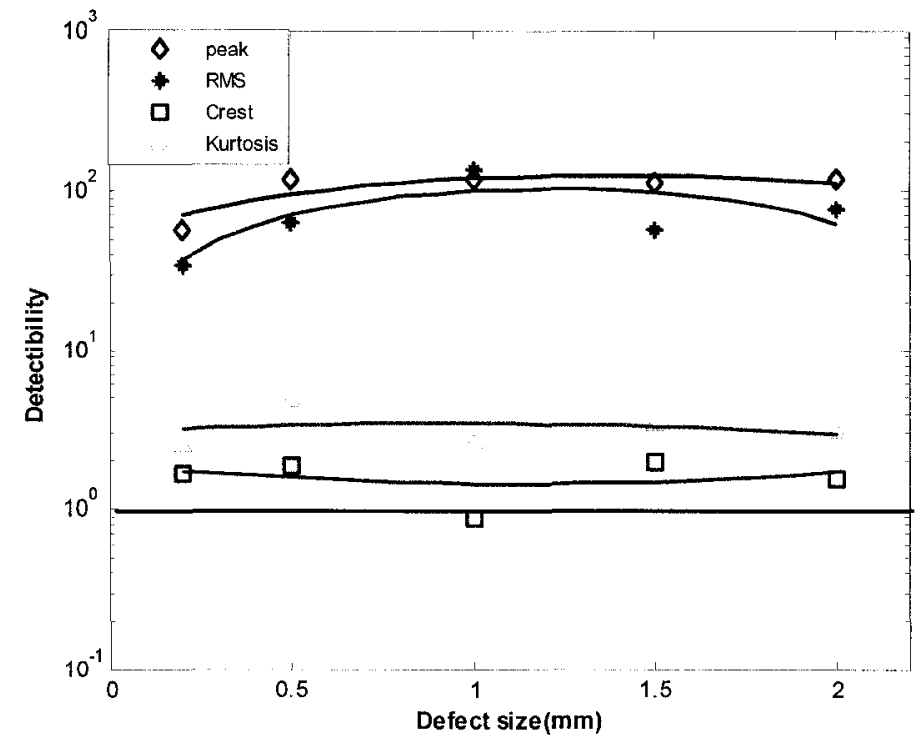

Figure 5.51 Defect detectability, acoustic signature (ACUT), roller, $1800 \mathrm{rpm}, 500 \mathrm{lbf}$

Similar to defective inner race, Eddy current and PUT time parameter did not show any sensitivity to defects on the bearing roller.

\subsubsection{Frequency-Domain Analysis}

Figures 5.52, 5.53, and 5.54 represent the spectra of the accelerometer, ACUT, and PUT signals for normal and defective bearings with defects on the rollers of $0.2,0.5$ and $1 \mathrm{~mm}$, respectively. In most of the defective cases, excitation frequencies are visible between 1200 to $3500 \mathrm{~Hz}$. In Figure 5.54, dominant spikes at the roller defect frequency, $f_{\text {ro }}$ of $187 \mathrm{~Hz}$ are barely present for each of the

defect sizes. Some harmonics of the shaft frequency are also noticed in the spectrum. 

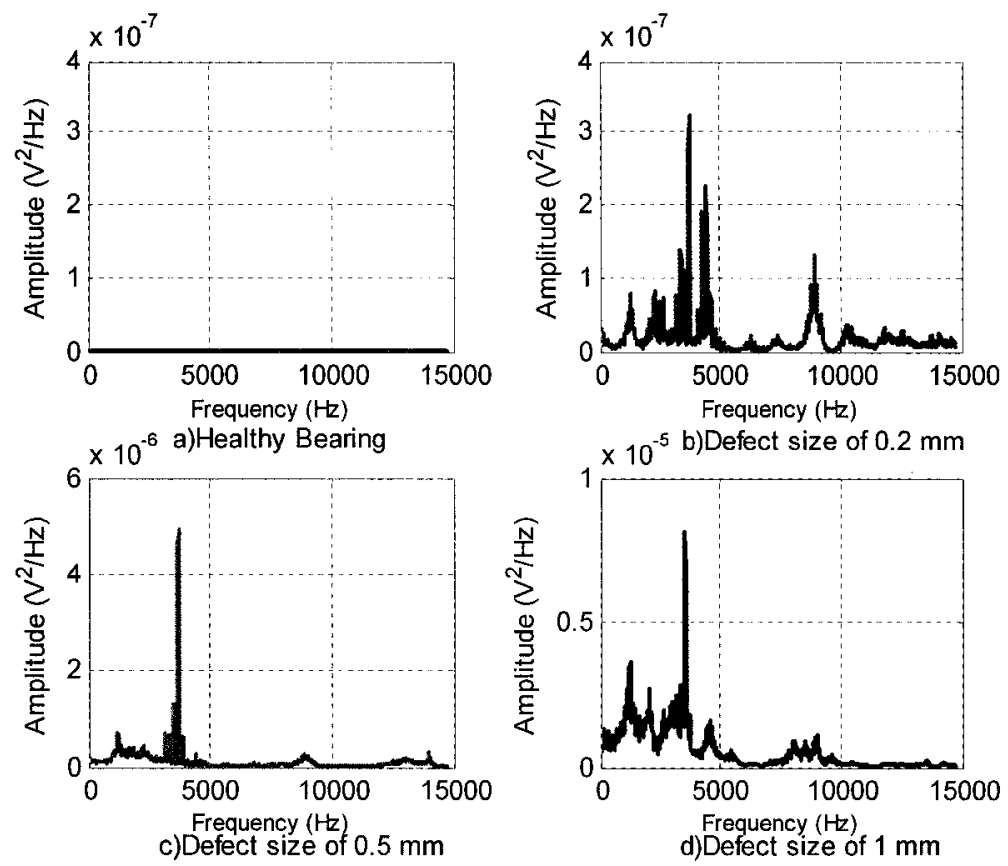

Figure 5.52 Vibration signature spectra of roller, 1800rpm, 500lbf
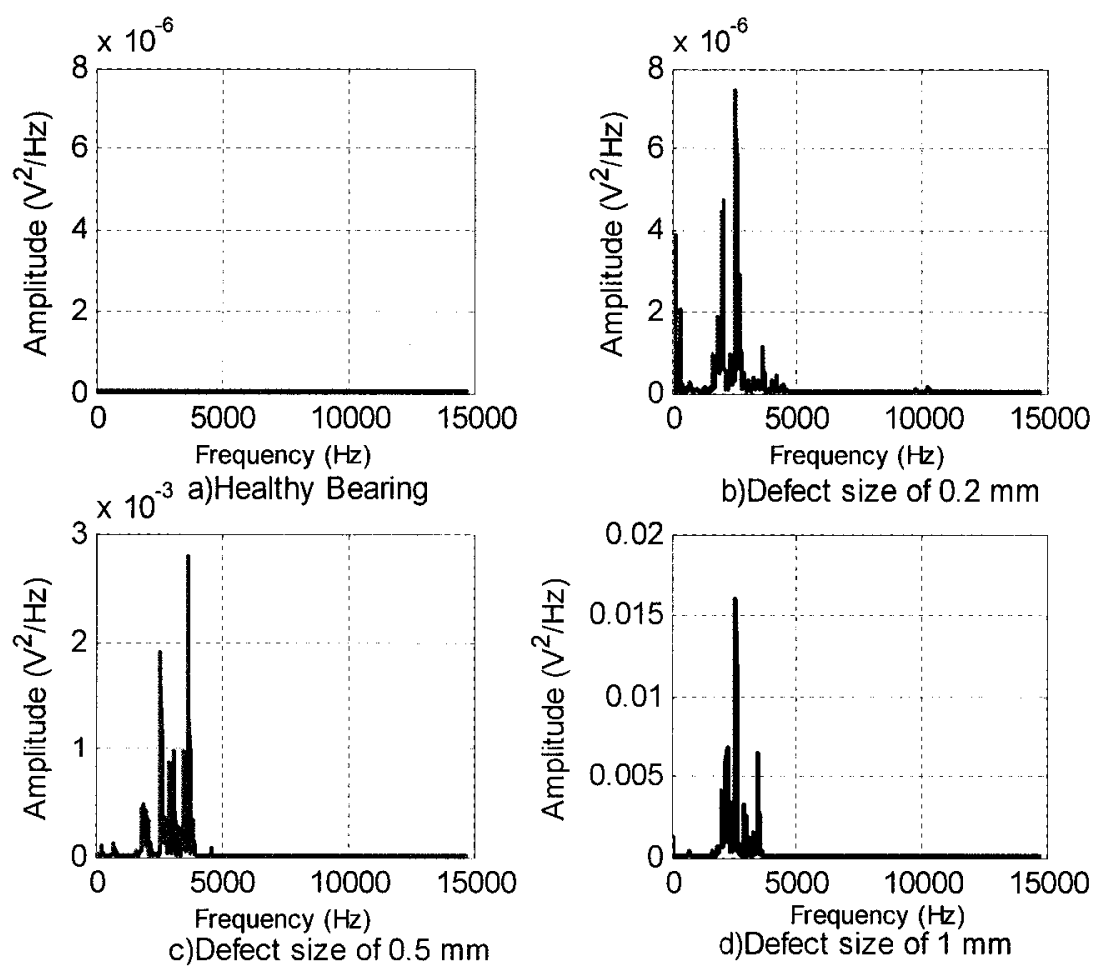

Figure 5.53 Acoustic signature (ACUT) spectra of roller, 1800rpm, 500lbf 

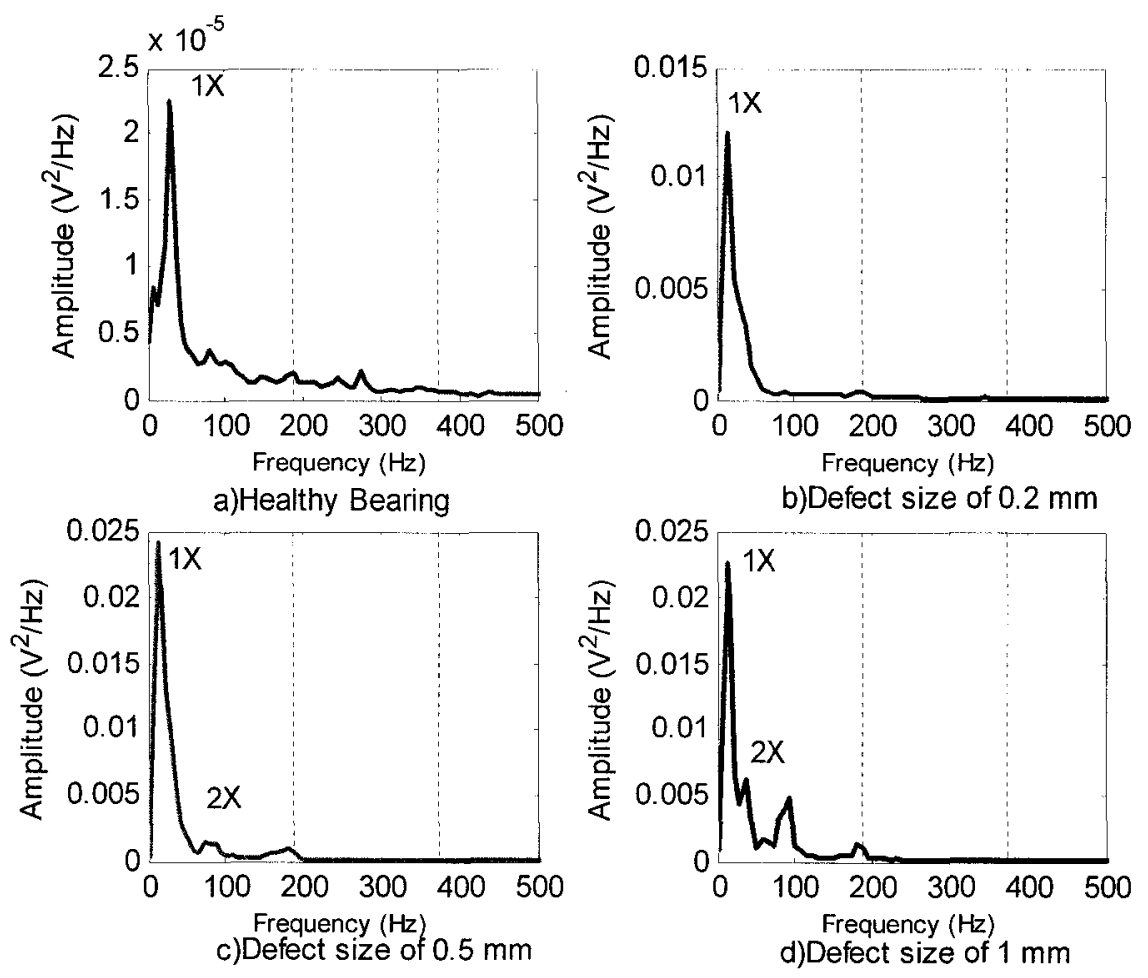

Figure 5.54 Acoustic signature (PUT) spectra of roller, 1800rpm, 500lbf

\subsubsection{Envelope Detection}

Figures 5.55, 5.56, and 5.57 represent the spectra of the demodulated accelerometer, ACUT, and Eddy current signals, respectively. Dominant spikes at the roller defect frequency $\left(\mathrm{F}_{\mathrm{ro}}\right)$ of $187 \mathrm{~Hz}$ and relatively low amplitude harmonics are clearly present with defects of size of $0.2,0.5$, and $1 \mathrm{~mm}$. It is important to note that, generally and based on the various operating conditions tested, defects on the roller were better identified with the ACUT than with the accelerometer. In Figure 5.57(b) (defect size of $0.2 \mathrm{~mm}$ ), there is no trace to the fault frequency. Moreover, the Eddy current probe was unable to detect $0.2 \mathrm{~mm}$ defect size on the roller for most operating conditions. However, the reason behind the nonsensitivity of the Eddy current probe to this defect size is not clear. The level of vibration generated by this defect could be just too low to be detected by the Eddy current sensor. The variable distance of the sensor to the bearing housing was also 
an issue that could have affected the probe reading.
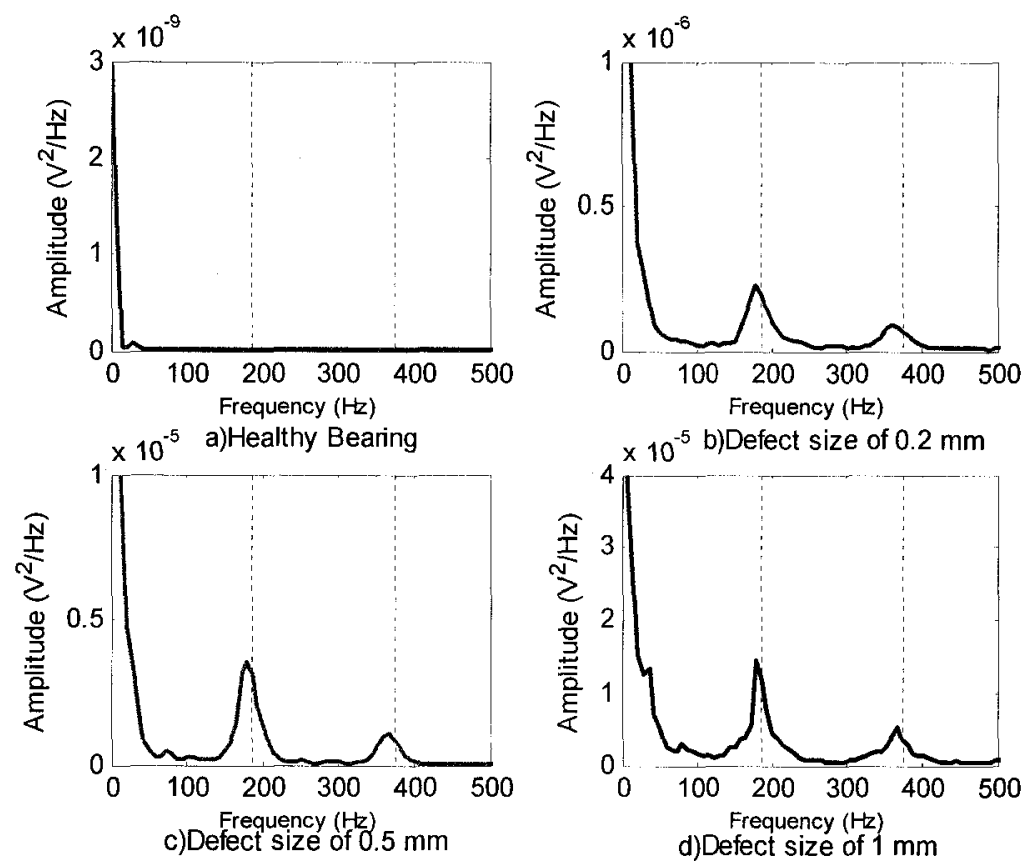

Figure 5.55 Demodulated vibration signature spectra of roller,

$1800 \mathrm{rpm}, 500 \mathrm{lbf}$
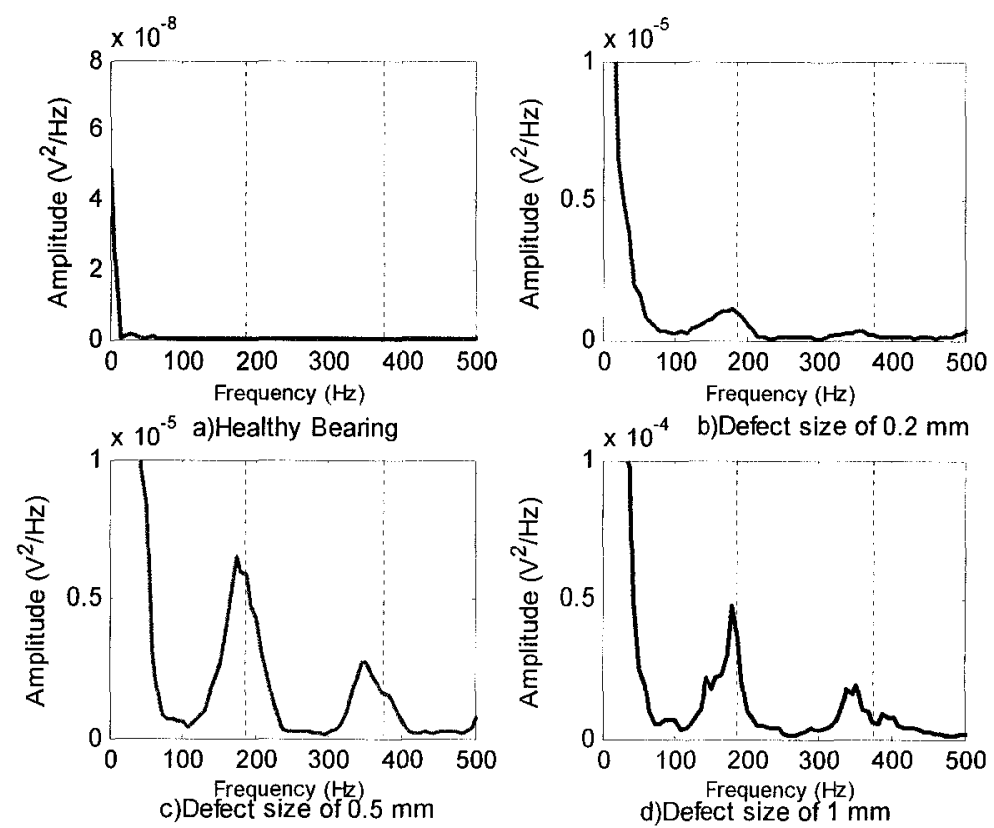

Figure 5.56 Demodulated acoustic signature (ACUT) spectra of roller, $1800 \mathrm{rpm}, 500 \mathrm{lbf}$ 

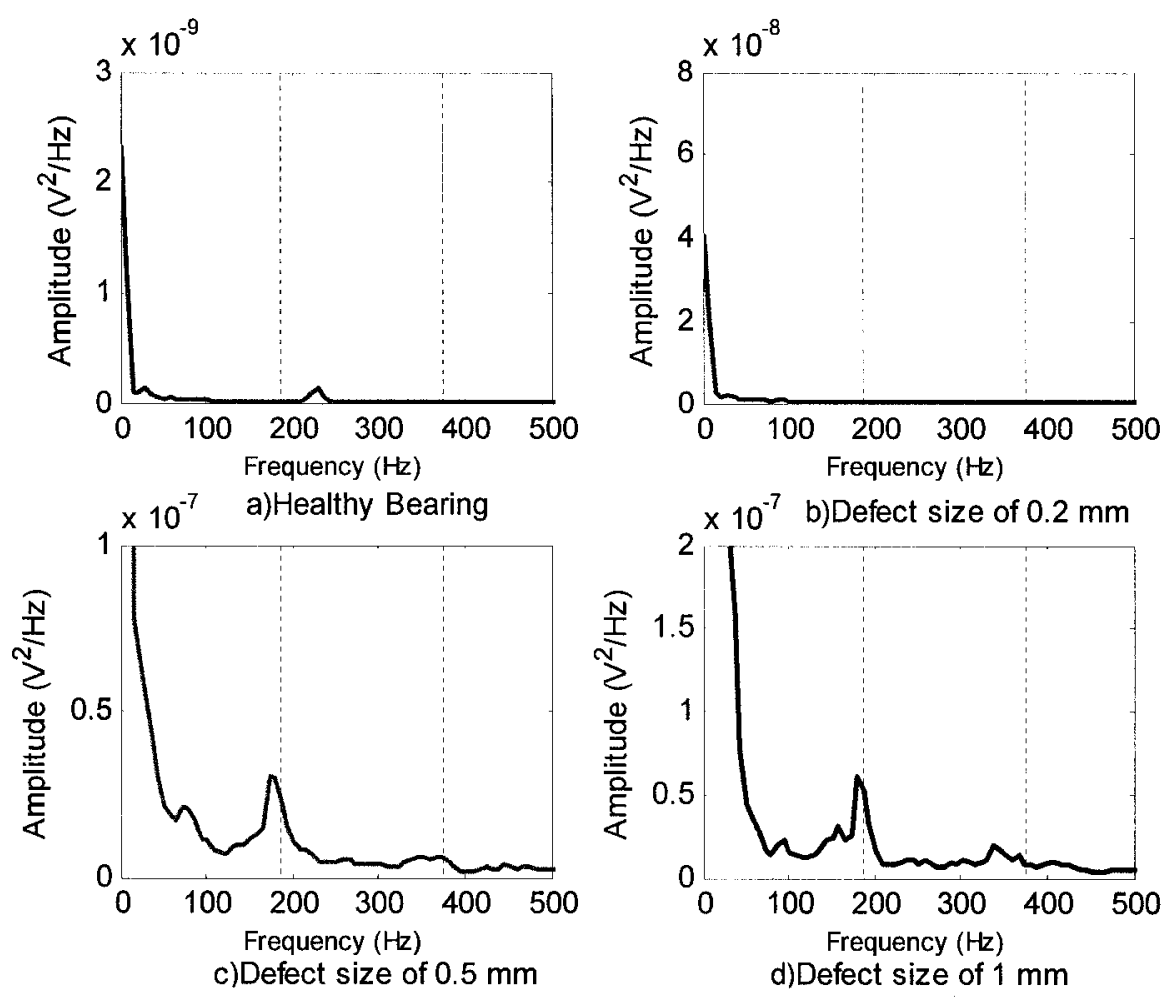

Figure 5.57 Demodulated Eddy current signal spectra of roller, $1800 \mathrm{rpm}, 500 \mathrm{lbf}$

\subsubsection{Frequency-Time Domain Analysis}

Figure 5.58 shows the results of the defect feature extraction from the ACUT signal for a defect size of $0.5 \mathrm{~mm}$ localized on the roller. As for the case of defective outer and inner races, the $7^{\text {th }}$ derivative of complex Gaussian wavelet was selected as the mother wavelet. Applying the Fourier transform to the wavelet-extracted signals results in the corresponding wavelet-based envelope spectra. The defect frequency is clearly visible on the frequency-scale distribution of the acoustic signature. The wavelet transform and its based envelop spectrum showed its relevance in detecting faulty rollers for all test conditions using the ACUT.

The time-frequency analysis of the radial and axial vibration signals was also successful to extract the defect features on the rolling element. 


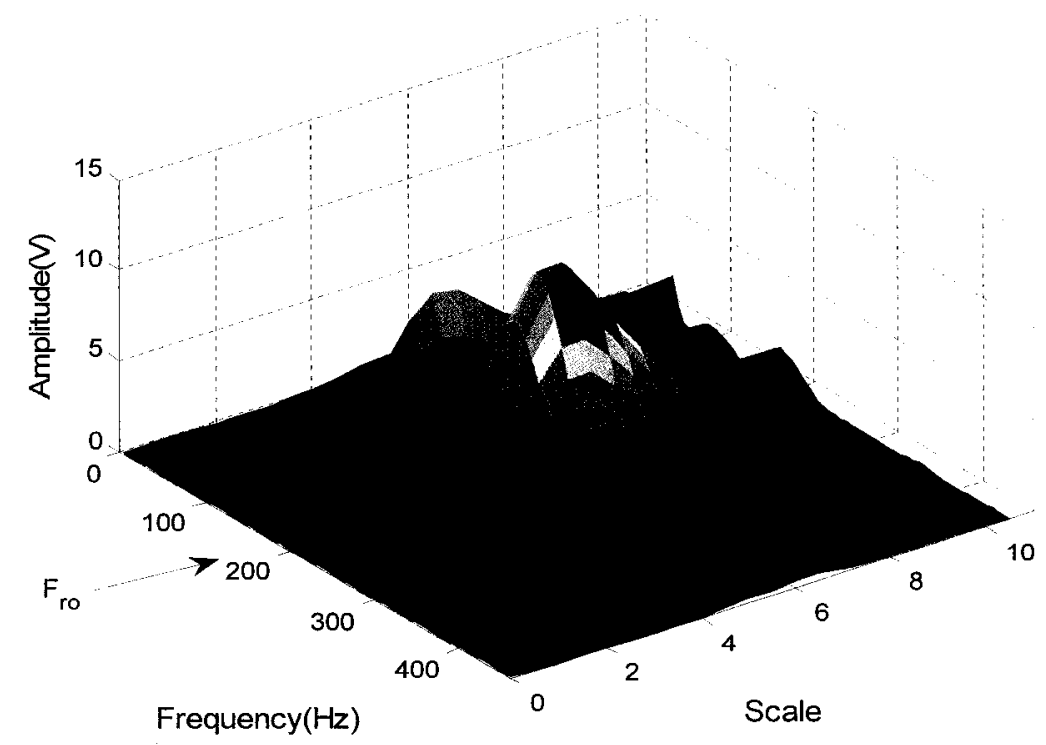

Figure 5.58 Wavelet-based envelope spectra of defective roller, 1800rpm, $500 \mathrm{lbf}$, acoustic signature, defect width of $0.5 \mathrm{~mm}$

\subsection{Conclusion remarks}

In this Chapter, a suite of sensors was evaluated using different processing techniques (time, frequency and time-frequency) for prognosis purposes of a defective roller bearing. Each of the applied sensors and techniques has the capability to detect defects localized on the bearing components. It was shown that because of the periodic behavior of the defective inner race signature under non-uniform load distribution, the bearing inner race represented the most challenging component, which detection faults on it was the most difficult task even using advanced signal processing techniques.

The success and capability of each sensor in detecting the presence of the simulated fatigue fault is stated below:

Accelerometer: represents the best sensor in identifying the bearing condition and tracking the fault size by using time, high frequency and time-frequency 
techniques.

Air Coupled Ultrasonic Transducer: shows significant and proper qualities for bearing defect diagnosis by using high frequency and time-frequency techniques.

Eddy Current: Except the installation issues, this sensor could be a good fault indicator using the high frequency resonance techniques.

Piezoelectric Ultrasonic Transducer: The only sensor, among the sensors suite, able to detect presence of faults on the bearing outer race directly in the frequency domain.

Piezo Strain gage: The output is very close to accelerometer's one. The sensor is capable of detecting large size faults localized on the outer race. 


\section{Chapter 6}

\section{Bearing Defect Classification}

In the previous Chapter, different techniques were applied and implemented to extract faults features from the various signals. Next step in this analysis would be automatically fault classification. A fault classifier is characterized by its capability to categorize the bearing as healthy or defected, identify the type of defect(s) as well as the level of the defect severity.

Early fault diagnosis improves the availability and performance of machinery operating with rolling bearings, while reducing the maintenance cost. The block diagram of Figure 6.1 shows the stages of a system from data collection to fault classification. Within this health management system, data fusion is utilized to combine information from multi-sensor data array and create useful features and then feed the features to a classifier.

A preliminary bearing fault classifier will be briefly described in this Chapter. The chosen data features, the classifier and the attempt to increase the performance of the system will also be discussed and analyzed. 


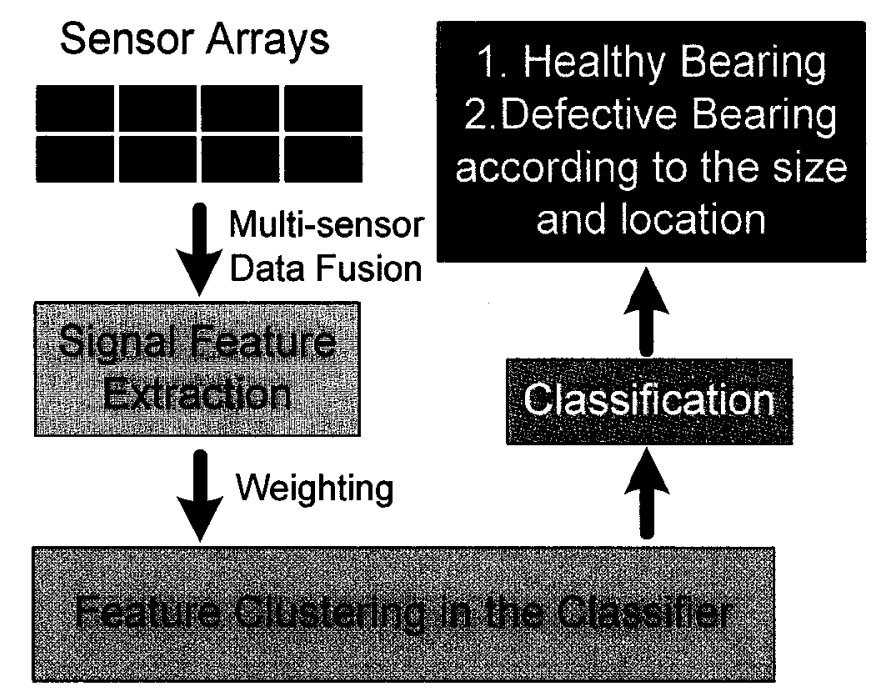

Figure 6.1 Classification method for fault diagnosis

\subsection{Data Fusion}

In a classifying space, data fusion plays a key role in classification and prognosis processes in terms of producing useful features, and combing features. Information fusion is the process of using joint information to reach a more confident decision about the condition of a bearing. Choosing the right sensors and extracting the right features are the bases of data fusion and condition monitoring system.

Since the performance of the classifier is closely linked to the quality of the features, it has been attempted to start taking account of as many feature extraction as to validate the sensitivity and reliability of the features and the classifier. Therefore, features from time, frequency, and wavelet domain as inputs to a bearing defect classification system have been selected. However, it should be mentioned that some parameters such as location of the sensors, test and ambient conditions may significantly affect the quality of the extracted features.

As shown in Table 6.1, 10 features have been extracted from each sensor including accelerometer (radial and axial), ACUT, Eddy current, and PUT for the defective bearing components with different defect widths and variable test 
conditions, based on the studies by researchers (Samanta, 2004), (Mahli, 2004), (Sun, 1999, 2004), and (Paya, 1997). These parameters are well known to be sensitive to changes in the bearing condition.

Table 6.1 Extracted features

\begin{tabular}{|l|l|l|}
\hline Time domain $(*)$ & Frequency domain $\left(^{*}\right)$ & Time-Frequency domain \\
\hline Peak value & Signal power @ $f_{b p o r}$ & $A_{c} @ f_{b p o r}$ \\
\hline RMS value & Signal power @ $f_{b p i r}$ & $A_{c} @ f_{b p i r}$ \\
\hline Crest factor & Signal power @ $f_{r o}$ & $A_{c} @ f_{r o}$ \\
\hline Kurtosis & $\begin{array}{l}\text { where: } A_{c}: \text { Amplitude of FFT of wavelet coefficients } \\
\left({ }^{*}\right) \text { Time and frequency domain metrics were obtained before and after HFRT for all sensors } \\
\text { except PUT }\end{array}$ \\
\hline
\end{tabular}

\subsection{Defect Classification}

Artificial Neural Network (ANN) has been the most common application in automated detection and diagnosis of machine conditions including bearing and even industrial processes. For example, Shao (2000) has shown the application of ANN to predict the remaining bearing life. However, the ANN model needs some trial and error choices such as the type of ANN, number of neurons, transfer functions, etc. Since the scope of this chapter is developing a primary model to classify the bearing defect based on the extracted different signals.

In order to evaluate the performance of the classification model on the performance of the bearing fault diagnosis, one of the available algorithms of decision trees in the WEKA software has been applied to the data. Decision trees are powerful and popular tools for classification and prediction. They represent sets of decisions structured as a tree shape. The attractiveness of decision trees is 
due to the fact that, in contrast to neural networks, decision trees represent rules. Rules can readily be expressed so that humans can understand them or even directly used in a database access language so that records falling into a particular category may be retrieved. The applied algorithm first analyzes the set of data by looking for specific patterns and trends. The algorithm then uses the results of this analysis to define the parameters of the model.

For this study, the cross-validation technique has been applied. It consists of dividing a data set $A$ into $n$ subsets $A_{i}$ and then running the given algorithm $n$ times, each time using a different set $A-A_{i}$ and validating the results on $A_{i}$.

16 different patterns were considered as output (healthy bearing, 5 different size defects on the outer race, 5 different size defects on the inner race, and 5 different size defects on the roller).

A combination of applied sensor's features was used to test the performance of the system. For all cases, the decision tree was used as the fault classifier. For example, Table 6.2 demonstrates the output matrix performance when attributes from radial accelerometer are the only inputs. When the defect size increases to 1 $\mathrm{mm}$, the classifier is barely able to diagnose the fault. The other sensor combinations show the same result.

In order to improve the accuracy of first attempt noted above, and the patterns of defect sizes of $1,1.5$ and $2 \mathrm{~mm}$ were compressed as one pattern which decreases the total classifier output to 10 patterns.

Table 6.3 indicates the other alternatives as input based on the sensors before and after combining the large defect sizes. Eddy current sensor gives the least performance. Such so, it has not been included to the other combinations as input.

The accuracy of the performance of the classifier based on the radial accelerometer features or ACUT ones is the same, which shows the possibility of replacing the accelerometer as a contact sensor to ACUT as a non contact sensor for classification purpose. It can also be seen that by increasing the number of 
input as sensor's distributors, the performance of the classifier increases to $70 \%$.

In addition, accuracy increases up to $80 \%$ as grouping the large defect sizes. 
Table 6.2 Classification results using a radial accelerometer

\begin{tabular}{|c|c|c|c|c|c|c|c|c|c|c|c|c|c|c|c|c|c|}
\hline \multirow[b]{2}{*}{ Condition } & \multirow{2}{*}{$\begin{array}{l}\text { Data } \\
\#\end{array}$} & \multicolumn{16}{|c|}{ Classifying results } \\
\hline & & $\overline{B I}$ & $\begin{array}{l}\mathrm{Ir}_{-} \\
0.2\end{array}$ & $\begin{array}{l}\mathrm{Ir}_{-} \\
0.5\end{array}$ & $\begin{array}{l}\mathbf{I r}_{-} \\
1\end{array}$ & $\begin{array}{l}\mathrm{Ir}_{-} \\
1.5\end{array}$ & $\begin{array}{l}\mathrm{Ir}_{-} \\
2\end{array}$ & $\begin{array}{l}\mathrm{Or}_{-} \\
0.2\end{array}$ & $\begin{array}{l}\mathrm{Or}_{-} \\
0.5\end{array}$ & $\begin{array}{l}\mathrm{Or}_{-} \\
1\end{array}$ & $\begin{array}{l}\mathrm{Or}_{-} \\
1.5\end{array}$ & $\begin{array}{l}\mathrm{Or}_{-} \\
2\end{array}$ & $\begin{array}{l}\text { Ro_ } \\
0.2\end{array}$ & $\begin{array}{l}\mathrm{Ro}_{-} \\
0.5\end{array}$ & $\begin{array}{l}\mathrm{Ro}_{-} \\
1\end{array}$ & $\begin{array}{l}\text { Ro_ } \\
1.5\end{array}$ & $\begin{array}{l}\text { Ro } \\
2\end{array}$ \\
\hline $\mathrm{Bl}$ & 27 & 26 & 1 & 0 & 0 & 0 & 0 & 0 & 0 & 0 & 0 & 0 & 0 & 0 & 0 & 0 & 0 \\
\hline Ir_0.2 & 9 & 0 & 8 & 0 & 0 & 0 & 0 & 0 & 0 & 0 & 0 & $\overline{0}$ & 1 & 0 & 0 & 0 & 0 \\
\hline Ir_0.5 & 9 & 0 & 1 & 3 & 1 & 0 & 0 & 0 & 0 & 0 & 0 & 0 & 0 & 3 & 0 & 0 & 1 \\
\hline Ir_1 & 9 & 0 & 0 & 1 & & & & 0 & 0 & 0 & 0 & $\overline{0}$ & 0 & 0 & 1 & 0 & 1 \\
\hline $\operatorname{Ir} 1.5$ & 9 & 0 & 0 & 0 & (2) & & 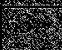 & 0 & 0 & 0 & 0 & 0 & 0 & 0 & 0 & 1 & 0 \\
\hline Ir_2 & 9 & 0 & 0 & 0 & (1) & & (6) & 0 & 0 & 0 & 0 & 0 & 0 & 0 & 1 & 2 & 1 \\
\hline Or_0.2 & 9 & 0 & 0 & 0 & 0 & 0 & 0 & 6 & 2 & 1 & 0 & 0 & 0 & 0 & 0 & 0 & 0 \\
\hline Or_0.5 & 9 & 0 & 0 & 0 & 0 & 0 & 0 & 0 & 5 & & $\ldots$ & & 0 & 0 & 0 & 0 & 0 \\
\hline Or_1 & 9 & 0 & 0 & 0 & 0 & 0 & 0 & 1 & 1 & & & & 0 & 0 & 0 & 0 & 0 \\
\hline Or_1.5 & 9 & 0 & 0 & 0 & 0 & 0 & 0 & 0 & 0 & & 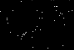 & & 0 & 0 & 0 & 0 & 0 \\
\hline Or_2 & 9 & 0 & 0 & 0 & 0 & 0 & 0 & 0 & 0 & 0 & 0 & 9 & 0 & 0 & 0 & 0 & 0 \\
\hline Ro_0.2 & 9 & 0 & 1 & 0 & 0 & 0 & 0 & 1 & 0 & 0 & 0 & 0 & 5 & 2 & 0 & 0 & 0 \\
\hline Ro_0.5 & 9 & 0 & 0 & 2 & 2 & 0 & 0 & 0 & 0 & 0 & 0 & 0 & 0 & 5 & 0 & 0 & 0 \\
\hline Ro_1 & 9 & 0 & 0 & 1 & 1 & 0 & 0 & 0 & 0 & 0 & 0 & 0 & 0 & 1 & & & \\
\hline Ro_1.5 & 9 & 0 & 0 & 0 & 1 & 2 & 3 & & 0 & 0 & 0 & 0 & 0 & 0 & & & \\
\hline Ro_2 & 9 & 0 & 0 & 1 & 1 & 1 & 1 & 0 & 0 & 0 & 0 & 0 & 0 & 1 & & & \\
\hline $\begin{array}{l}\text { Where: } \\
\text { Bl: health } \\
0.2,0.5,1 \text {, }\end{array}$ & $\begin{array}{l}\text { bearing } \\
\text { and }\end{array}$ & .04 & .110 & 1 & 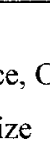 & t & 政 & 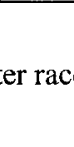 & $\mathrm{NO}$ & 年 & TH & & & & & & \\
\hline
\end{tabular}


Table 6.3 Performance results

\begin{tabular}{|c|c|c|}
\hline \multirow[b]{2}{*}{ Sensor(s) } & \multicolumn{2}{|c|}{ Accuracy $(\%)$} \\
\hline & Group1 & Group2 \\
\hline $\mathrm{S}_{1}$ & 56 & 70 \\
\hline $\mathrm{S}_{2}$ & 55 & 68 \\
\hline $\mathrm{S}_{3}$ & 56 & 72 \\
\hline $\mathrm{S}_{4}$ & 28 & 41 \\
\hline $\mathrm{S}_{5}$ & 52 & 64 \\
\hline $\mathrm{S}_{1}, \mathrm{~S}_{2}$ & 58 & 68 \\
\hline $\mathrm{S}_{1}, \mathrm{~S}_{3}$ & 62 & 78 \\
\hline $\mathrm{S}_{1}, \mathrm{~S}_{2}, \mathrm{~S}_{3}$ & 68 & 80 \\
\hline $\mathrm{S}_{1}, \mathrm{~S}_{3}, \mathrm{~S}_{5}$ & 65 & 77 \\
\hline $\mathrm{S}_{1}, \mathrm{~S}_{2}, \mathrm{~S}_{3}, \mathrm{~S}_{5}$ & 70 & 80 \\
\hline \multicolumn{3}{|l|}{ where: } \\
\hline \multirow{2}{*}{\multicolumn{3}{|c|}{$\mathrm{S}_{1}$ : Radial accelerometer, $\mathrm{S}_{2}$ : axial accelerometer, }} \\
\hline & & \\
\hline \multicolumn{3}{|c|}{ Grop 1: Before merging the large defect sizes } \\
\hline \multicolumn{3}{|c|}{ Group2: After merging the large defect sizes } \\
\hline
\end{tabular}

\subsection{Summary}

A simple bearing fault diagnosis system has been built up. The signal features based on the behavior of the faulty bearing were extracted. The data were fed to the system, which has performed effectively. The results showed that the decision tree algorithm is effective for bearing fault classification and diagnosis and can give satisfactory results with an accuracy rate up to $70 \%$. Grouping the defect size of each component showed that the decision tree represents a more promising scheme in the case where incipient faulty bearings data is available. The effectiveness of the number of applied sensors has been investigated and the accuracy rate depended on the number of the sensors. 


\section{Chapter 7}

\section{Conclusions and Future work}

The ability of vibration and acoustic sensors for detection and diagnosis of a defective bearing has been investigated using various processing techniques.

During this research the following proceedings were performed:

i. A review of failure modes of rolling elements bearings, the consequences of failures, the procedures that could be used to detect these faults and the fault signatures was conducted. A general model of faulty bearing vibration was developed.

ii. The available signal analysis techniques for bearing defect detection were reviewed and analyzed. The review showed that techniques based on time frequency (especially wavelet analysis) and frequency analysis are the most appropriate diagnosis techniques for faulty bearings, respectively.

iii. To provide healthy and faulty bearing under desired running conditions, a test rig was built and simulated faults on the bearing components were created. The test rig is located at the NRC campus (IAR/GTL). Six different sensors, mainly vibration and acoustic emission, were evaluated and most of the data were collected simultaneously. Impact tests were also performed to identify the bearing natural frequencies especially the defect-induced ones. 
iv. The application of the selected signal analysis techniques to the acquired signals showed that:

a. The vibration and acoustic (ACUT) time domain features (RMS and peak values) identify the fault on the outer race more prominently as load, speed and/or defect size increase. Except for the crest factor, all other parameters were sensitive to the progress of the defect size in the inner race and roller with vibration and acoustic (ACUT) signatures. In general, eddy current probe and PUT were unable to track the progress of the defect size using time domain attributes.

b. PUT was the only sensor, which could detect the defect as small as $0.2 \mathrm{~mm}$ on the outer race with the Fourier series bases spectrum.

c. The HFRT technique applied to vibration, acoustic (ACUT), eddy current and strain gage signals provided reliable fault diagnosis on the faulty bearing and the amplitudes of the fault frequency were significantly higher than those obtained from the healthy bearing. However, for the defective inner race, the interpretation of the results was, some times, confusing and could be misleading especially for smaller defects.

d. In the time-frequency distributions, the presence of background noise while increasing the shaft speed was clearly identified. However, the influence of the load on the radial vibration and acoustic distribution were not very significant.

e. Using wavelet-based envelope spectrum, most bearing fault related components could be identified in the frequency-scale distribution of vibration and acoustic signatures. The amplitude at the specific defect frequency increases with the defect size. Wavelet-based technique has continuity in frequency, which 
allows investigating small frequency ranges in detail and is accurate in analyzing the faulty bearing signals.

f. Comparatively, vibration and acoustic signatures were the most sensitive to the detection of bearing faults.

g. The results demonstrated that selecting the appropriate signal processing technique could significantly affect the defect identification and consequently, improve the reliability of the bearing health monitoring.

v. The signals' features (time, frequency, and wavelet analysis), which contain important information regarding the bearing condition were extracted. A practical classification model based on a decision tree algorithm was developed and applied on the extracted feature. Overall, the results showed that by monitoring the bearing with more than one sensor the performance of the classifying system was increased by $14 \%$. The results also showed that the defect could be detected at an early stage on each component of the bearing. Accelerometer and ACUT gave the better diagnosis performance, respectively.

As it is possible to obtain several faulty bearing data to understand the bearing behavior, the bearing dynamic model can be tuned and used independently of experimental data. This model allows the prediction of results of running condition such as very high speed and load for which the test rig is not designed.

In time-frequency analysis, further works is needed to optimize the number of applied scales on the signals.

Discrete wavelet transform of Eddy current and PUT data may also provide information on defective bearings.

Signal feature selection is an effective prerequisite in classification. Therefore, 
more interpretation of extracted features is still required. Investigation of other pattern recognition techniques based on the various signal features to identify the location and size of the defect on a bearing may increase the fault identification and classification rate. As the bearing fault diagnosis technology improves, it will allow the rotary machineries to work efficiently and safely. 


\section{Chapter 8}

\section{References}

Akagaki T., Nakamura M., Monzen T., Kawabata M., "Analysis of the behavior of rolling bearings in contaminated oil using some condition monitoring techniques", Journal of Tribology engineering, 2006,220(5),pp 447-453.

Berry J. E., "How to track rolling element bearing health with vibration signature analysis". Journal of sound and vibration, Nov. 1991, pp 24-35.

Chopra I., "Review of Current Status of Smart Structure and Integrated Systems", SPIE smart structures and integrated systems, 1996,2217,pp 20-62.

Choundhury A. and Tandon N., "Application of acoustic emission technique for the detection of defects in rolling element bearings", Tribology International, 2000, 33, pp 39-45.

Cohen A., "Wavelets and multi-scale signal processing", Chapman and Hall. 1995.

Cohen L., " Time-Frequency distributions-A review", proceeding of the IEEE, 1989,177(7), pp 941-981.

Dadouche A., Rezaei A., Wickramasinghe V., Dmochowski V., Bird J., and Nitzsche F. "Sensitivity of Air-Coupled Ultrasound and Eddy Current sensors to bearing fault detection", $62^{\text {nd }}$ STLE meeting and exhibition ,2007.

Dadouche A., Safizadeh M. S., Bird J., Dmochowski W., and Forsyth D.S., "Development of PHM Methods for Roller Bearings Phase 1: Evaluation of the 
NDE techniques applicable to bearing condition monitoring", Internal report, 2005.

Dadouche A., Safizadeh M. S., Bird J., Dmochowski W. and Forsyth D.S., "A comparative study of air-coupled ultrasound sensor and accelerometer in detecting bearing defects," in the proceedings of the World Tribology Congress III, 2005, Washington, paper number WTC2005-64282.

Dadouche, A. and Bird, J., "Techniques and sensors for bearing fault detection: Literature analysis and technological needs", Internal report, LTR-GTL-2006$0002,2004$.

Daubechies I., "Ten lectures on wavelets", Publisher: Society for Industrial and Applied Mathematics, 1992.

Dyer D., Stewart R.M., “ Detection of rolling Element bearing damaged by statistical Vibration Analysis", Journal of mechanical Design, 1978, 100(2), pp 229-235.

Forrester B. D., "Use of the Wigner-Ville distribution in helicopter transmission fault detection". Proceeding of Australian symposium on signal processing and applications, Adelaide, Australia, 1989,pp78-82.

Harris T.A., "Rolling bearing analysis", New York: John Wiley, 2000.

Howard I., "A review of rolling element bearing vibration - detection, diagnosis and prognosis", Defense science and technology organization technical report DSTO-RR-0013, Australia, 1994.

Hubbard, B. B., "The world according to wavelets: The story of a mathematical technique in the making", AK Peters Ltd. Wellesley, MA, 1996.

Mahli A., Gao R.X., "PCA-based feature selection scheme for machine defect classification", IEEE transaction on instrumentation and measurement, 2004, 
53(6), pp1517-1525.

Mallat S., " A wavelet tour of signal processing". San Diego: Academic Press, 1998.

Mathews J. R., " The use of Acoustic Emission for bearing defect identification and estimation of defect size ", Gordon and Breach Science Publishers Inc., New York. ISSN 0730-7152,1983.

McFadden P. D. and Smith J. D., "Vibration monitoring of rolling element bearings by the high frequency resonance technique: a review," Tribology International, 1984, 17 (1), pp 3-10.

Meyer Y.,"Wavelets: algorithms and applications", Publisher: Society for Industrial and Applied Mathematics, 1993.

Miettinen J. and Andersson, P., "Acoustic emission of rolling bearings lubricated with contaminated grease", Tribology International, 2000, 33(11), pp743-802.

Mitchell, John S., "Introduction to machinery analysis and monitoring", Penn Well publishing company, Tulsa, Oklahoma, 1994.

Newland D.E., "Wavelet analysis of vibration, part I: Theory", Journal of Vibration and Acoustics, ASME 1994,116,pp 409-416.

Nishio K., Hoshiya S, Miyachi T. "An investigation of the early detection of defects in ball bearings by the vibration monitoring", ASME, 1979.

Oppenheim A. V., Schafer R. W. "Discrete-time signal processing", Englewood Cliffs, N. J: Prentice Hall, 1989.

Paya B.A., East III, Badi M.N.M., "Artificial neural network based fault diagnostics of rotating machinery using wavelet transforms as a preprocessor", $\mathrm{J}$ Mechanical. System. Signal processing 1997,11(5), pp 751-65. 
Qiu H., Lee J., Lin J., and Yu G., "Wavelet filter-based weak signature detection method and its application on rolling element bearing prognostics," Journal of Sound and Vibration, 2006, 289(4-5), pp 1066-1090.

Randall R. B., Antoni J., and Chobsaard S., "The relationship between spectral correlation and envelope analysis in the diagnostics of bearing faults and other cyclostationary machine signals," Mechanical Systems and Signal Processing, 2001, 15(5), pp 945-962.

Rief Z., Lai MS. "Detection of developing bearing failures by means of vibration". ASME, Design Engineering Division, 1998,18(1), pp 231-236.

Rubini R. and Meneghetti U., "Application of the envelope and wavelet transform analyses for the diagnosis of incipient faults in ball bearings," Mechanical Systems and Signal Processing, 2001,15(2), pp 287-302.

Samanta B., Khamis R., Al-Balushi, Al-Araimi S., "Bearing Fault Detection Using Artificial Neural Networks and Genetic Algorithm", Journal of Applied Signal Processing, 2004,2004 (3), pp 366-377.

Schnidel D.W, Bashford A.G. and Hutchins D.A., "Chromatic aberration of an air-coupled ultrasonic Fresnel zone-plate," IEEE Transactions on Ultrasonics, Ferroelectrics and Frequency Control, 1999, 46(1), pp.242-246.

Schnidel D.W. and Hutchins D.A, "Air-Coupled Ultrasonic Transducer," U.S. Patent 5, Feb. 1994.

Shao Y. and Nezu K.,"Prognosis of remaining bearing life using neural networks," Journal of System and Control Engineering, 2000, 214(3), pp 217 230.

Shi D. F., Wang W. J., and Qu L. S., "Defect detection for bearings using envelope spectra of wavelet transform," ASME Journal of Vibration and Acoustics, 2004, 126(4), pp 567-573. 
Shiroishi J., Li Y., Liang S., Kurfess T. and Danyluk S., "Bearing condition diagnostics via vibration and acoustic emission measurements," Mechanical Systems and Signal Processing, 1997, 11 (5), pp 693-705.

Stoica, P., and Moses R.L.," Introduction to spectral analysis," Prentice-Hall, Englewood Cliffs, NJ, 1997.

$\mathrm{Su}$ Y., and Lin S.J., "On initial fault detection on a tapered roller bearing: frequency domain analysis", Journal of Sound and Vibration, 1992, 155(1), pp7584.

Sun Q., Tang Y., Lu W., "Feature extraction and classification for drill wear monitoring", The $14^{\text {th }}$ international conference on flexible automation and intelligent manufacturing, Toronto, 2004.

Sun Q., Xi F., Chen P., Krishnappa G., "Bearing condition monitoring through pattern recognition analysis". The $6^{\text {th }}$ international conference on sound and vibration, Denmark, 1999.

Swansson, N.S. and Favaloro, S.C." Application of vibration analysis to the condition monitoring", Aero Propulsion report, Aeronautical research laboratory, 1984.

Tandon N. and Nakra B.C., "Comparison of vibration and acoustic measurement techniques for the condition monitoring of rolling element bearings", Tribiology International, 1992, pp 205-212.

Tandon, N., "A comparison of some vibration parameters for the condition monitoring of rolling element bearings", Measurements 12,1994, pp285-289.

Vive A. A., " Piezoelectric transducers and application”, Springer-Verlag, Berlin Heidelberg. 2004.

Westbrook M.H. and Turner J.D., "Automotive sensors", Bristol, Philadelphia, 
Institute of Physics Pub, 1994.

White M., "Rolling Element Bearing Vibration Transfer Characteristics: effect of stiffness", ASME, Journal of Applied Mechanics, 1979,46, pp 677-684.

White, C.J., "Detection of gearbox failure", Workshop of on-condition maintenance, Institute of sound and vibration research, 1972. 
Appendix A: Axial Accelerometer Time and Frequency-Domain
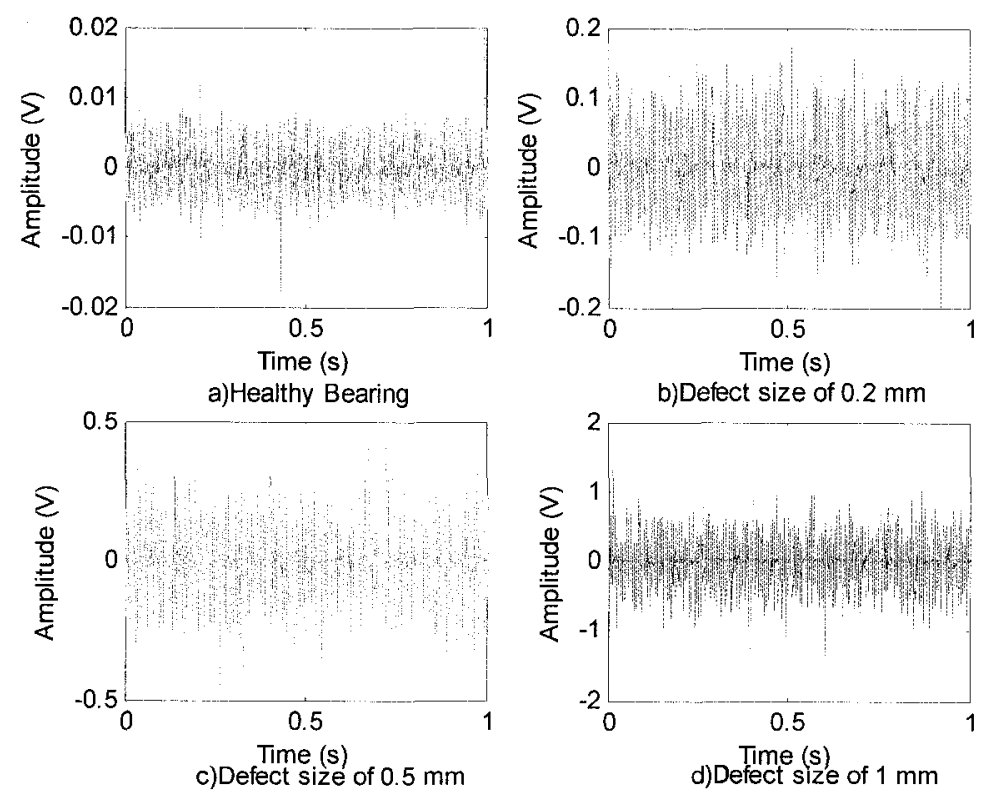

Figure A.1 Axial accelerometer signal for outer race, 1800rpm, 500lbf
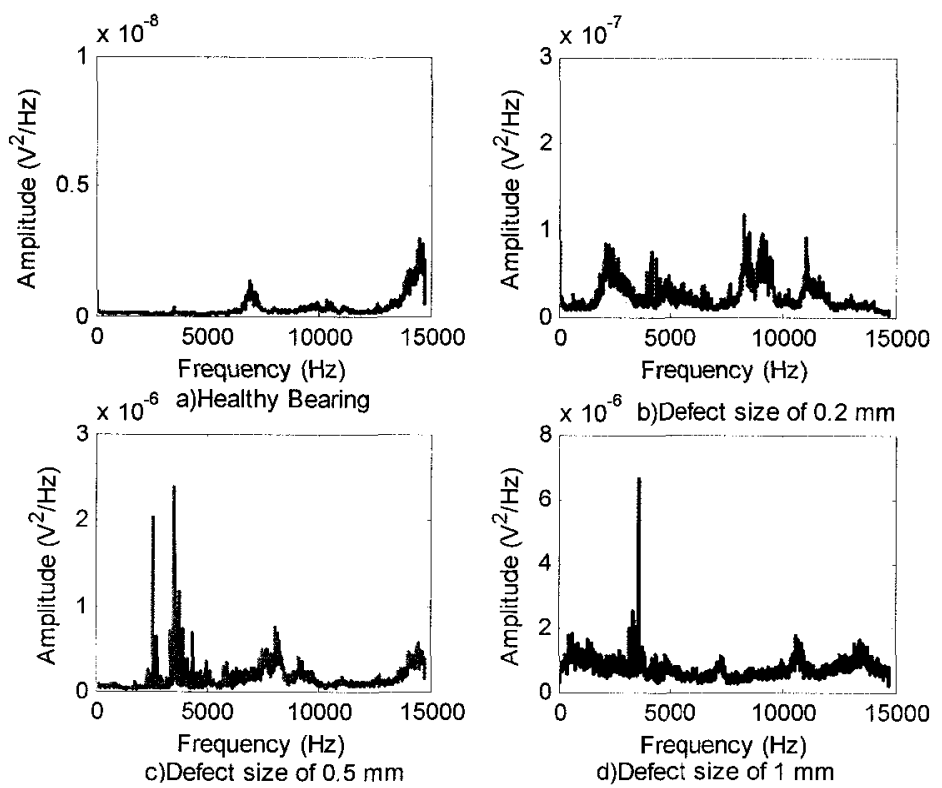

Figure A.2 Axial accelerometer spectra of outer race, 1800rpm, 500lbf 

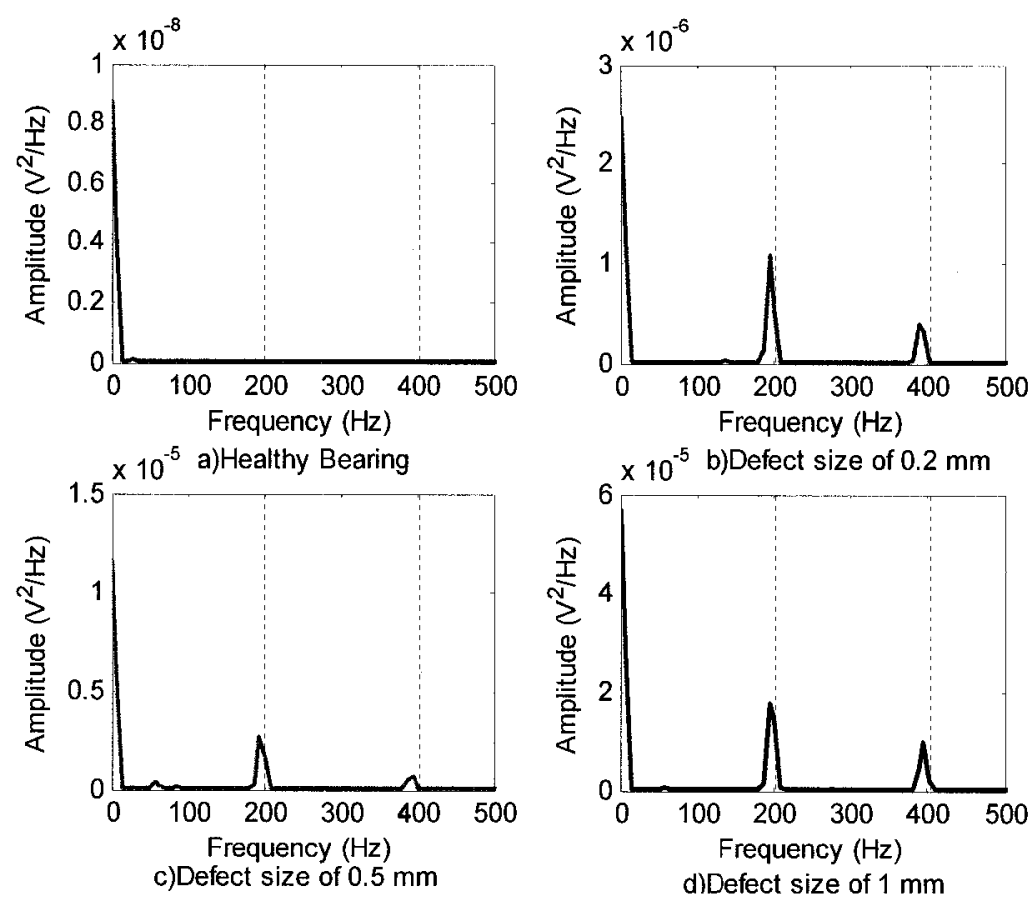

Figure A.3 Accelerometer demodulated signal spectra of outer race,

1800rpm, 5001bf 